\title{
Regio- and Stereoselective Hydrosilylation of Unsymmetrical Alkynes Catalyzed by a Well-defined Low-valent Cobalt Catalyst
}

\author{
Alejandro Rivera-Hernández, ¥ Brendan J. Fallon, ¥ Sandrine Ventre, Cédric Simon, Marie- \\ Hélène Tremblay, Geoffrey Gontard, Etienne Derat, Muriel Amatore, Corinne Aubert, \\ Marc Petit* \\ UPMC UNIV Paris 06, Institut Parisien de Chimie Moléculaire, UMR CNRS 8232, Case 229, 4 Place \\ Jussieu, 75252 Paris Cedex 05, France.
}

\section{List of contents:}

$\begin{array}{ll}\text { 1. General remarks } & \mathrm{S} 1\end{array}$

2. Ratios of the hydrosilylation products of unsymmetrical internal alkynes S2

3. General procedures $\quad \mathrm{S} 3$

4. Spectroscopic characterization $\quad$ S4

$\begin{array}{lll}\text { 5. Preparation of dihydrido cobalt(III) complex } 6 & \text { S19 }\end{array}$

$\begin{array}{ll}\text { 6. Theoretical methods } & \text { S21 }\end{array}$

$\begin{array}{ll}\text { 7. X-ray data } & \text { S36 }\end{array}$

$\begin{array}{lr}\text { 8. References } & \text { S39 }\end{array}$

9. NMR spectra $\quad$ S40 


\section{General remarks}

Commercial reagents were purified prior to use following the guidelines of Perrin and Armarego. ${ }^{1}$ Toluene was purified by mean of distillation under dry nitrogen atmosphere on benzophenone/sodium ketyl and degassed by sparging argon. Organic solutions were concentrated under reduced pressure on a Büchi rotary evaporatory. Chromatographic purifications of products were accomplished using force-flow chromatography on Davisil (LC60A) SI $60 \AA(40-63 \mu \mathrm{m})$ silica gel according to the method of Still. ${ }^{2}$ Thin layer chromatography (TLC) was performed on Merck 60 F254 silica gel plates. TLC visualization was performed by fluorescence quenching $(\lambda=254 \mathrm{~nm})$, dipping in $\mathrm{KMnO} 4$ or paranisaldehyde stains. Filtrations through Celite were performed using Hyflo Super Cel from Fluka. ${ }^{1} \mathrm{H}$ NMR spectra were recorded on a Brucker 400 AVANCE or 300 AVANCE (400 and $400 \mathrm{Mhz}$ respectively) and are referenced relative to residual $\mathrm{CDCl}_{3}$ protons signals at $\delta 7.26 \mathrm{ppm} .{ }^{13} \mathrm{C} \mathrm{NMR}$ spectra were recorded on a Brucker 400 AVANCE or 300 AVANCE (100 and $100 \mathrm{Mhz}$ respectively) and are referenced relative to $\mathrm{CDCl}_{3}$ at $\delta 77.00 \mathrm{ppm}$. Data are reported as follows: chemical shift $(\delta \mathrm{ppm})$, multiplicity (s = singlet, $\mathrm{d}=$ doublet, $\mathrm{t}=$ triplet, $\mathrm{q}=$ quartet, $\mathrm{qt}=$ quintuplet, $\mathrm{m}=$ multiplet, $\mathrm{bs}=$ broad signal), coupling constant $(\mathrm{Hz})$ and integration. IR spectra were recorded on a Bruker Tensor 27 (ATR diamond) and are reported in terms of frequency of absorption $\left(\mathrm{cm}^{-1}\right)$. High-resolution mass spectra were obtained from the Laboratoire Structure et Fonction de Molécules Bioactives (Université Pierre et Marie Curie, Paris 6). 


\section{Ratios of the hydrosilylation products of unsymmetrical internal alkynes}

\section{Table S1. $E / Z$ and $3 / 3^{\prime}$ adduct ratios ${ }^{a, b, c}$}

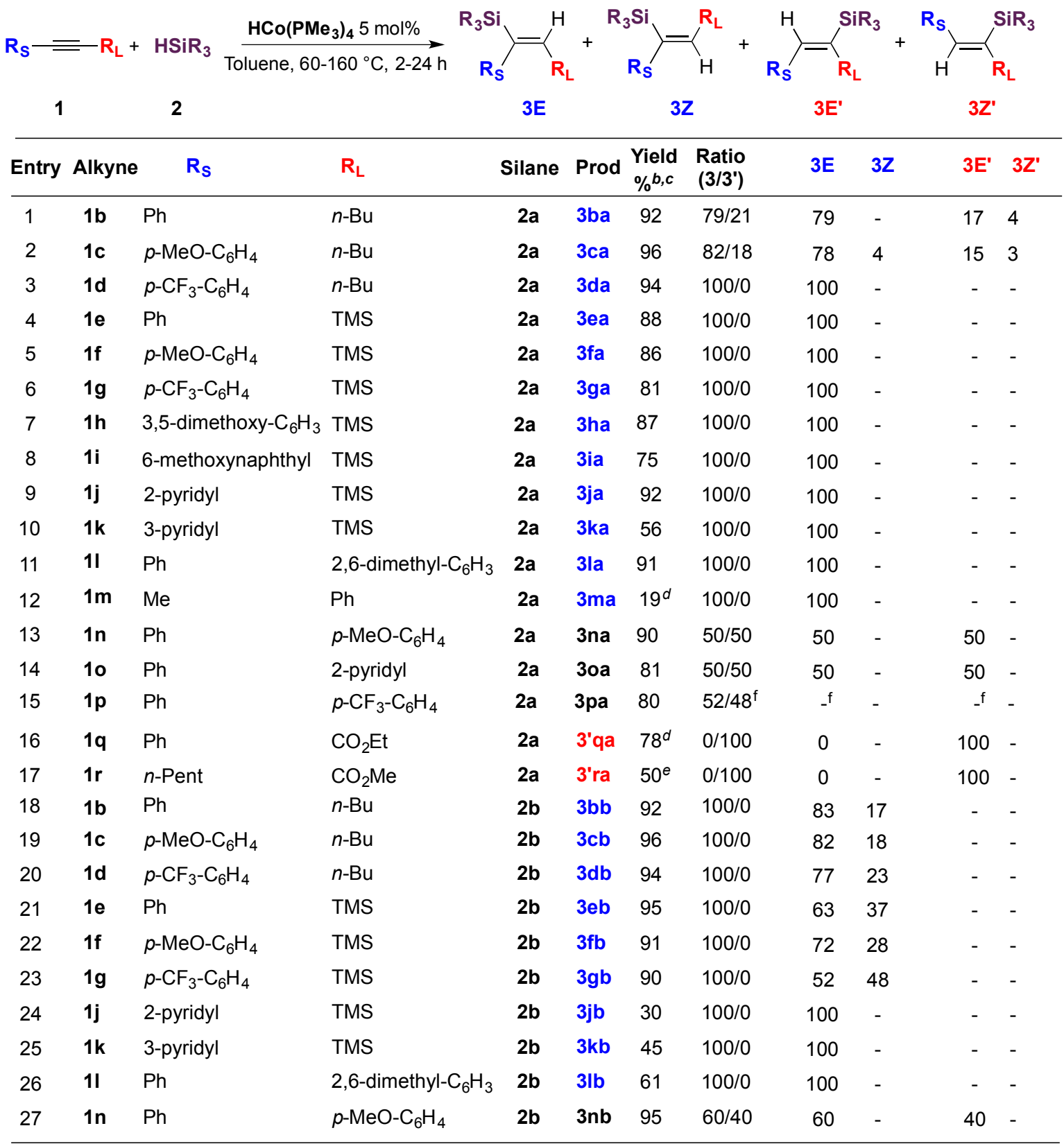

${ }^{a}$ Alkyne $(0.5 \mathrm{mmol}), \mathrm{HSiR}_{3}(0.55 \mathrm{mmol}) .{ }^{b}$ Isolated yields. ${ }^{c}$ For detailed information regarding the $(E / Z)$ and $(\alpha / \beta)$ ratios of the obtained products, see SI. ${ }^{d}$ Reaction was performed at $60{ }^{\circ} \mathrm{C}$ for $24 \mathrm{~h} .{ }^{e}$ Reaction was performed at $100{ }^{\circ} \mathrm{C}$ for $24 \mathrm{~h} .{ }^{f}$ Obtained as a mixture of $3 \mathrm{E}$ and $3 \mathrm{E}^{\prime}$ (the Major compound was not determined). 


\section{General Procedures}

\section{General procedure A: hydrosilylation of diphenylacetylene}

Under argon, a glass vial $(0.5-2 \mathrm{~mL})$ was charged with diphenylacetylene $(89 \mathrm{mg}, 0.5 \mathrm{mmol}$ ) and the cobalt catalyst $9.1 \mathrm{mg}, 0.025 \mathrm{mmol}$ of $\left.\mathrm{HCo}\left(\mathrm{PMe}_{3}\right)_{4}\right)$. The vial was sealed followed by the addition of freshly distilled and degassed toluene $(1 \mathrm{ml})$ and the corresponding silane $(0.55 \mathrm{mmol})$. The reaction was heated in an oil bath at $110{ }^{\circ} \mathrm{C}$ for $24 \mathrm{~h}$. The reaction mixture was diluted with ethyl acetate $(2 \mathrm{~mL})$, filtered through a plug of celite and washed with ethyl acetate $(3 \times 10 \mathrm{ml})$. The crude product was purified by silica chromatography (mixtures of pentane/ethyl acetate as eluent) to afford the final products.

\section{General procedure B: hydrosilylation of unsymmetric alkynes with triphenylsilane}

Under argon, a glass vial $(0.5-2 \mathrm{~mL})$ was charged with $\left.\mathrm{HCo}\left(\mathrm{PMe}_{3}\right)_{4}\right)(9.1 \mathrm{mg}, 0.025 \mathrm{mmol})$ and triphenylsilane $(0.55 \mathrm{mmol})$ and then sealed. Next, it was added freshly distilled and degassed toluene $(1 \mathrm{ml})$ and the corresponding alkyne $(0.5 \mathrm{mmol})$. The reaction was heated in an oil bath at $160{ }^{\circ} \mathrm{C}$ for $2 \mathrm{~h}$. The reaction mixture was diluted with ethyl acetate $(2 \mathrm{~mL})$, filtered through a plug of celite and washed with ethyl acetate $(3 \times 10 \mathrm{ml})$. The crude product was purified by silica chromatography (mixtures of pentane/ethyl acetate as eluent) to afford the final products.

\section{General procedure C: hydrosilylation of unsymmetric alkynes with triethoxysilane}

Under argon, a glass vial $(0.5-2 \mathrm{~mL})$ was charged with $\left.\mathrm{HCo}\left(\mathrm{PMe}_{3}\right)_{4}\right)(9.1 \mathrm{mg}, 0.025 \mathrm{mmol})$ and then sealed. Next, it was added freshly distilled and degassed toluene $(1 \mathrm{ml})$, the corresponding alkyne $(0.5 \mathrm{mmol})$ and triethoxysilane $(0.55 \mathrm{mmol})$. The reaction was heated in an oil bath at $160{ }^{\circ} \mathrm{C}$ for $2 \mathrm{~h}$. The reaction mixture was diluted with ethyl acetate $(2 \mathrm{~mL})$, filtered through a plug of celite and washed with ethyl acetate $(3 \times 10 \mathrm{ml})$. The crude product was purified by silica chromatography (mixtures of pentane/ethyl acetate as eluent) to afford the final products. 


\section{Spectroscopic characterization.}

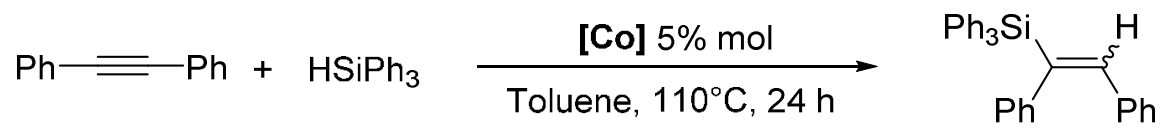

\section{Triphenyl-[(E)-(1,2-diphenylvinyl)]silane (3aa) ${ }^{3}$}

The general procedure A was applied to diphenylacetylene $1 \mathrm{a}(89 \mathrm{mg}, 0.5 \mathrm{mmol}$ ) and triphenylsilane $2 \mathrm{a}(143 \mathrm{mg}, 0.55 \mathrm{mmol})$ in degassed toluene $(1 \mathrm{ml})$ then heated in an oil bath at 110 ${ }^{\circ} \mathrm{C}$ for $24 \mathrm{~h}$. Purification by silica gel flash-chromatography afforded (pentane/ethyl acetate 99:1) the title compound as a white solid (173 mg, 79\% yield) as a 96/4 mixture of $E / Z$ isomers. ${ }^{1} \mathbf{H}$ NMR $\left(400 \mathrm{MHz}, \mathrm{CDCl}_{3}\right) \delta 7.52-7.26(\mathrm{~m}, 15 \mathrm{H}), 7.19-7.08(\mathrm{~m}, 6 \mathrm{H}), 7.05(\mathrm{~s}, 1 \mathrm{H}, \mathrm{C}=\mathrm{CH}) 7.01-6.88(\mathrm{~m}$, 4H). ${ }^{13} \mathrm{C}$ NMR (100 MHz, $\left.\mathrm{CDCl}_{3}\right) \delta 143.3,141.8,141.0,137.1,136.5,133.8,129.7,129.5,128.4$, $128.3,127.9,127.7,127.4,125.9$.

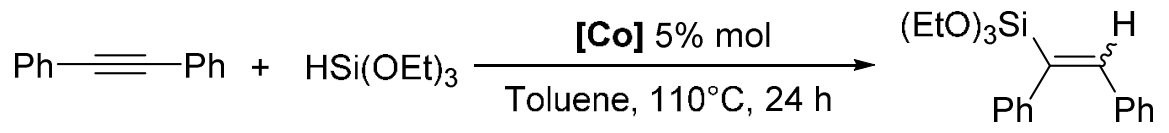

\section{Triethoxy-[(E)-(1,2-diphenylvinyl)]silane (3ab) ${ }^{4}$}

The general procedure A was applied to diphenylacetylene $1 \mathrm{a}(89 \mathrm{mg}, 0.5 \mathrm{mmol}$ ) and triethoxysilane $\mathbf{2 b}(0.1 \mathrm{~mL}, 0.55 \mathrm{mmol})$ in degassed toluene $(1 \mathrm{ml})$ then heated in an oil bath at 110 ${ }^{\circ} \mathrm{C}$ for $24 \mathrm{~h}$. Purification by silica gel flash-chromatography (pentane/ethyl acetate 95:5) afforded the title compound as a colorless oil (155 mg, 91\% yield) as a 98/2 mixture of $E / Z$ isomers. ${ }^{1} \mathbf{H}$ NMR $\left(400 \mathrm{MHz} \mathrm{CDCl}_{3}\right) \delta$ 7.32-7.07 (m, $11 \mathrm{H}$, phenyl- $\mathrm{H}$ and $\left.\mathrm{C}=\mathrm{CH}\right), 3.86(\mathrm{q}, J=7.0 \mathrm{~Hz}, 3 \mathrm{H}), 1.23(\mathrm{t}, J=7.0$ $\mathrm{Hz}, 5 \mathrm{H}) .{ }^{13} \mathrm{C}$ NMR $\left(100 \mathrm{MHz} \mathrm{CDCl}_{3}\right) \delta 142.4,140.7,137.1,136.9,129.8,128.4,128.2,127.8,127.4$, $126.0,58.8,18.1$.

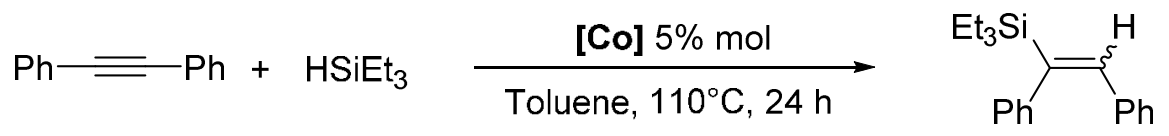

\section{Triethyl-[(E)-(1,2-diphenylvinyl)]silane (3ac) ${ }^{5}$}

The general procedure A was applied to diphenylacetylene $1 \mathrm{a}(89 \mathrm{mg}, 0.5 \mathrm{mmol}$ ) and triethylsilane 2c $(0.09 \mathrm{~mL}, 0.55 \mathrm{mmol})$ in degassed toluene $(1 \mathrm{ml})$ then heated in an oil bath at $110{ }^{\circ} \mathrm{C}$ for $24 \mathrm{~h}$. Purification by silica gel flash-chromatography (pentane/ethyl acetate 99:1) afforded the title compound as a colorless oil ( $85 \mathrm{mg}, 58 \%$ yield) as a mixture of $E / Z$ isomers $>98 E$. ${ }^{1} \mathbf{H}$ NMR (400 $\left.\mathrm{MHz}, \mathrm{CDCl}_{3}\right) \delta 7.24-7.08(\mathrm{~m}, 3 \mathrm{H}), 7.03-6.84(\mathrm{~m}, 7 \mathrm{H}), 6.70(\mathrm{~s}, 1 \mathrm{H}, \mathrm{C}=\mathrm{CH}), 0.88(\mathrm{t}, J=7.9 \mathrm{~Hz}, 9 \mathrm{H})$, $0.57(\mathrm{q}, J=7.8 \mathrm{~Hz}, 6 \mathrm{H}) .{ }^{13} \mathrm{C}$ NMR $\left(100 \mathrm{MHz} \mathrm{CDCl}_{3}\right) \delta 144.1,143.1,138.7,137.4,129.5,128.6$, $127.8,127.3,126.9,125.5,7.3,2.8$.

Minor product: triethyl-[(Z)-(1,2-diphenylvinyl)]silane signals are overlapped with the $E$-isomer. 


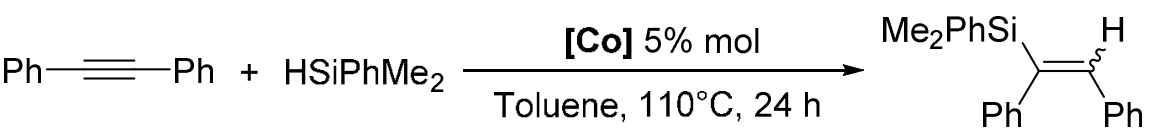

\section{Dimethylphenyl-[(E)-(1,2-diphenylvinyl)]silane (3ad) ${ }^{6}$}

The general procedure A was applied to diphenylacetylene 1a $(89 \mathrm{mg}, 0.5 \mathrm{mmol}$ ) and dimethylphenylsilane $\mathbf{2 d}(0.08 \mathrm{~mL}, 0.55 \mathrm{mmol})$ in degassed toluene $(1 \mathrm{ml})$ then heated in an oil bath at $110{ }^{\circ} \mathrm{C}$ for $24 \mathrm{~h}$. Purification by silica gel flash-chromatography (pentane/ethyl acetate 99:1) afforded the title compound as a yellow oil $(147 \mathrm{mg}, 94 \%$ yield as a mixture of $E / Z$ isomers $>98 E$. ${ }^{1} \mathrm{H}$ NMR $\left(400 \mathrm{MHz}, \mathrm{CDCl}_{3}\right) \delta 7.66-7.62(\mathrm{~m}, 2 \mathrm{H}), 7.47-7.41(\mathrm{~m}, 3 \mathrm{H}), 7.35-7.23(\mathrm{~m}, 4 \mathrm{H}), 7.18-$ $7.13(\mathrm{~m}, 3 \mathrm{H}), 7.06-6.97(\mathrm{~m}, 4 \mathrm{H}), 6.93(\mathrm{~s}, 1 \mathrm{H}, \mathrm{C}=\mathrm{CH}), 0.49(\mathrm{~s}, 6 \mathrm{H}) .{ }^{13} \mathrm{C}$ NMR (100 MHz, CDCl $)_{3} \delta$ $145.4,142.7,139.6,138.1,137.7,134.7$ (2C), 130.0, 129.5, 129.0, 128.3, 128.2, 128.1, $127.6(2 \mathrm{C})$, $126.1,-2.5$.

Minor product: dimethylphenyl-[(Z)-(1,2-diphenylvinyl)]silane signals are overlapped with the $E$ isomer.

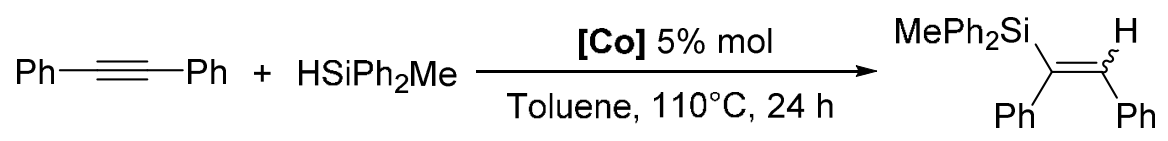

\section{Methyldiphenyl-[(E)-(1,2-diphenylvinyl)]silane (3ae) ${ }^{7}$}

The general procedure A was applied to diphenylacetylene $1 \mathrm{a}(89 \mathrm{mg}, 0.5 \mathrm{mmol}$ ) and methyldiphenylsilane $2 \mathrm{e}(0.11 \mathrm{~mL}, 0.55 \mathrm{mmol})$ in degassed toluene $(1 \mathrm{ml})$ then heated in an oil bath at $110^{\circ} \mathrm{C}$ for $24 \mathrm{~h}$. Purification by silica gel flash-chromatography (pentane/ethyl acetate 99:1) afforded the title compound as a yellow solid ( $162 \mathrm{mg}, 86 \%$ yield) as a mixture of $E / Z$ isomers $>98$ E. ${ }^{1} \mathrm{H}$ NMR (400 MHz, $\left.\mathrm{CDCl}_{3}\right) \delta 7.68-7.63(\mathrm{~m}, 4 \mathrm{H}), 7.49-7.40(\mathrm{~m}, 6 \mathrm{H}), 7.29-7.19(\mathrm{~m}, 3 \mathrm{H}), 7.16-$ $7.11(\mathrm{~m}, 3 \mathrm{H}), 7.04-6.98(\mathrm{~m}, 4 \mathrm{H}), 6.92(\mathrm{~s}, 1 \mathrm{H}, \mathrm{C}=\mathrm{CH}), 0.64(\mathrm{~s}, 3 \mathrm{H}) .{ }^{13} \mathrm{C}$ NMR (100 MHz, CDCl $) \delta$ 142.6, 142.1, 141.9, 137.1, 135.7, 135.3 (2C), 129.7, 129.4, 128.5, 127.9, 127.9, 127.8, 127.3 (2C), $125.8,-3.9$.

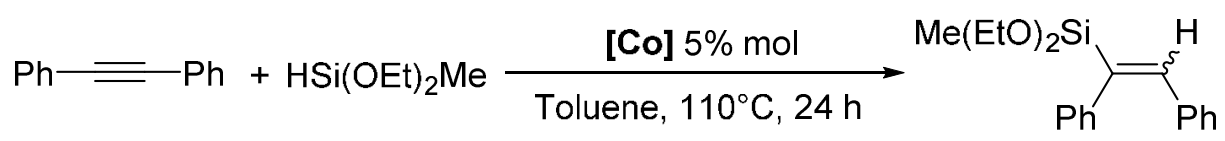

\section{Methyldiethoxy-[(E)-(1,2-diphenylvinyl)]silane (3af)}

The general procedure A was applied to diphenylacetylene $1 \mathrm{a}(89 \mathrm{mg}, 0.5 \mathrm{mmol}$ ) and methyldiethoxysilane $2 \mathbf{f}(0.09 \mathrm{~mL}, 0.55 \mathrm{mmol})$ in degassed toluene $(1 \mathrm{ml})$ then heated in an oil bath at $110{ }^{\circ} \mathrm{C}$ for $24 \mathrm{~h}$. Purification by silica gel flash-chromatography (pentane/ethyl acetate 95:5) afforded the title compound as a yellow oil (103 $\mathrm{mg}, 66 \%$ yield) as a $98 / 2$ mixture of $E / Z$ isomers. ${ }^{1} \mathbf{H}$ NMR $\left(400 \mathrm{MHz} \mathrm{CDCl}_{3}\right) \delta 7.34-7.29,7.25-7.20,7.17-7.11,7.08-7.02(\mathrm{~m}, 11 \mathrm{H}$, phenyl- $\mathrm{H}$ and $\mathrm{C}=\mathrm{CH}$ ), 3.85 (qd, $J=7.0,1.2 \mathrm{~Hz}, 4 \mathrm{H}), 1.25(\mathrm{t}, J=7.0 \mathrm{~Hz}, 6 \mathrm{H}), 0.23(\mathrm{~s}, 3 \mathrm{H}) .{ }^{13} \mathrm{C} \mathbf{N M R}(100 \mathrm{MHz}$, $\left.\mathrm{CDCl}_{3}\right) \delta 141.2,141.0,140.6,136.9,129.8,128.5,127.9,127.8,127.3,125.9,58.5,18.3,-4.8$. IR 
(film, cm ${ }^{-1}$ ): v 2972.15, 2877.80, 1600.86, 1073.07. HRMS (ESI) Calculated for $\mathrm{C}_{19} \mathrm{H}_{24} \mathrm{NaO}_{2} \mathrm{Si}$ $[\mathrm{M}+\mathrm{Na}]^{+}$335.1438, measured 335.1450.

$$
\mathrm{Ph}=\mathrm{Ph}+\mathrm{H}\left(i-\mathrm{Pr}_{2}\right) \mathrm{Si}-\mathrm{C} \equiv \mathrm{C}-\mathrm{SiMe}_{3}
$$

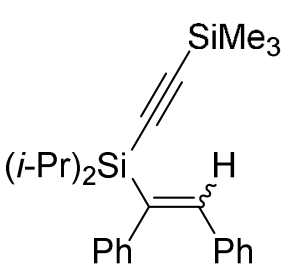

\section{Diisopropyl[(trimethylsilyl)ethynyl][(E)-(1,2-diphenylvinyl)]silane (3ag)}

The general procedure A was applied to diphenylacetylene 1a $(89 \mathrm{mg}, 0.5 \mathrm{mmol}$ ) and diisopropyl[(trimethylsilyl)ethynyl]silane ${ }^{8} \mathbf{2 g}(117 \mathrm{mg}, 0.55 \mathrm{mmol})$ in degassed toluene $(1 \mathrm{ml})$ then heated in an oil bath at $110{ }^{\circ} \mathrm{C}$ for $24 \mathrm{~h}$. Purification by silica gel flash-chromatography (pentane/ethyl acetate 99:1) afforded the title compound as a yellow oil (181 $\mathrm{mg}, 93 \%$ yield) as a mixture of $E / Z$ isomers $>98 E .{ }^{1} \mathbf{H} \mathbf{N M R}\left(400 \mathbf{~ M H z}, \mathrm{CDCl}_{3}\right) \delta 7.31-7.24,7.23-7.17,7.13-7.07$, $7.01-6.96(\mathrm{~m}, 11 \mathrm{H}$, phenyl- $H$ and $\mathrm{C}=\mathrm{CH}), 1.17-1.09(\mathrm{~m}, 2 \mathrm{H}), 1.10(\mathrm{~d}, J=6.7 \mathrm{~Hz}, 6 \mathrm{H}), 1.06(\mathrm{~d}, J=$ $6.7 \mathrm{~Hz}, 6 \mathrm{H}), 0.23(\mathrm{~s}, 9 \mathrm{H}) .{ }^{13} \mathrm{C}$ NMR $\left(100 \mathrm{MHz} \mathrm{CDCl}_{3}\right) \delta 142.4,141.9,140.0,137.3,129.7,128.5$, 128.0, 127.8, 127.1, 125.9, 118.3, 108.5, 18.0, 17.9, 11.6, 0.0. IR (film, $\mathrm{cm}^{-1}$ ): v 2954.48, 2862.10, 1599.74, 1249.13. HRMS (ESI) Calculated for $\mathrm{C}_{25} \mathrm{H}_{34} \mathrm{NaSi}_{2}[\mathrm{M}+\mathrm{Na}]^{+} 413.2091$, measured 413.2077.

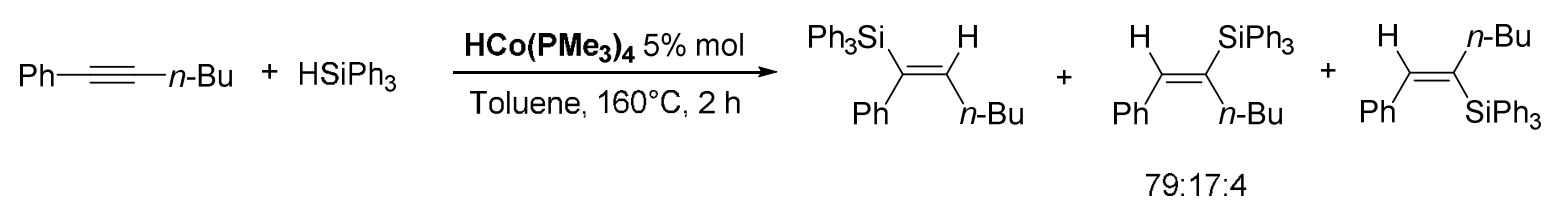

\section{Triphenyl-[(E)-(1-phenyl-2-butylvinyl)]silane (3ba)}

The general procedure B was applied to 1-phenyl-1-hexyne $1 \mathrm{~b}(87 \mathrm{mg}, 0.5 \mathrm{mmol}$ ) and triphenylsilane $(143 \mathrm{mg}, 0.55 \mathrm{mmol})$ in degassed toluene $(1 \mathrm{ml})$ then heated in an oil bath at $160{ }^{\circ} \mathrm{C}$ for $2 \mathrm{~h}$. Purification by silica gel flash-chromatography (pentane/ethyl acetate 99:1) afforded the title compound as a colorless oil (192 mg, 92\% yield). ${ }^{1} \mathbf{H} \mathbf{N M R}\left(400 \mathrm{MHz}, \mathrm{CDCl}_{3}\right) \delta$ 7.32-6.77 (m, phenyl-H), $6.12(\mathrm{t}, J=7.1 \mathrm{~Hz}, \mathrm{C}=\mathrm{CH}), 1.98\left(\mathrm{q}, J=7.2 \mathrm{~Hz}, 2 \mathrm{H}, \mathrm{C}=\mathrm{CH}-\mathrm{CH}_{2^{-}}\right), 1.20\left(\mathrm{~m},-\mathrm{CH}_{2^{-}}-\mathrm{CH}_{2^{-}}\right), 0.72(\mathrm{t}$, $\left.J=7.1 \mathrm{~Hz}, 3 \mathrm{H}, \mathrm{CH}_{2}-\mathrm{CH}_{3}\right) .{ }^{13} \mathrm{C}$ NMR $\left(100 \mathrm{MHz} \mathrm{CDCl}_{3}\right) \delta 148.5,141.7,138.6,136.5,136.4,136.2$, 135.8, 134.4, 129.3, 128.7, 128.0, 127.8, 127.8, 127.6, 125.5, 31.6, 30.1, 22.3, 13.9. IR (film, cm ${ }^{-1}$ ): v 3068.07, 3049.52, 2956.03, 2923.23, 2359.54, 1427.87. HRMS (ESI) Calculated for $\mathrm{C}_{30} \mathrm{H}_{30} \mathrm{NaSi}$ $[\mathrm{M}+\mathrm{Na}]^{+}$441.2009, measured 441.2001.

Minor product: triphenyl-[(E)-1-butylvinyl-2-phenyl)]silane relevant signal: ${ }^{\mathbf{1}} \mathbf{H}$ NMR (400 $\mathbf{~ M h z}$, $\left.\mathrm{CDCl}_{3}\right) \delta 2.35\left(\mathrm{~m}, \mathrm{C}=\mathrm{C}(\mathrm{Si})-\mathrm{CH}_{2^{-}}\right)$. Other signals are overlapped with the major product. 
Minor product: triphenyl-[(Z)-1-butylvinyl-2-phenyl)]silane relevant signal: ${ }^{\mathbf{1}} \mathbf{H}$ NMR (400 $\mathbf{M h z}$, $\left.\mathrm{CDCl}_{3}\right) \delta 2.58\left(\mathrm{~m}, \mathrm{C}=\mathrm{C}(\mathrm{Si})-\mathrm{CH}_{2^{-}}\right)$. Other signals are overlapped with the major product.

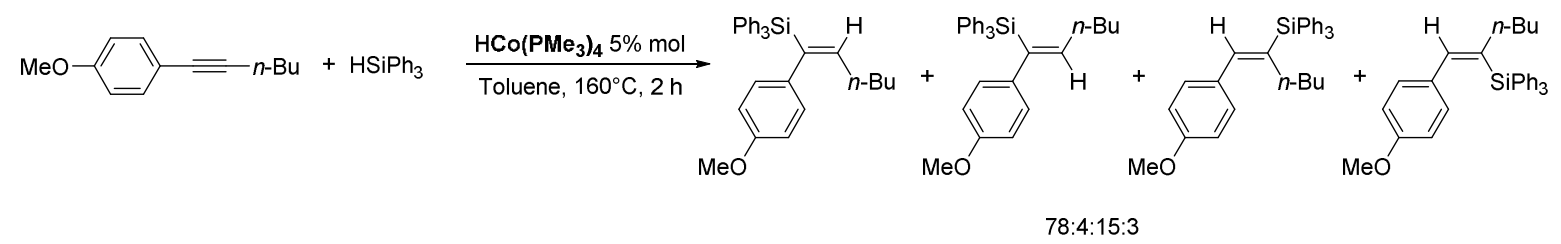

\section{Triphenyl-[(E)-(1-(4-methoxyphenyl)-2-butylvinyl)]silane (3ca)}

The general procedure B was applied to 1-(4-methoxyphenyl)-1-hexyne 1c (94 mg, $0.5 \mathrm{mmol}$ ) and triphenylsilane $(143 \mathrm{mg}, 0.55 \mathrm{mmol})$ in degassed toluene $(1 \mathrm{ml})$ then heated in an oil bath at $160{ }^{\circ} \mathrm{C}$ for $2 \mathrm{~h}$. Purification by silica gel flash-chromatography (pentane/ethyl acetate 95:5) afforded the title compound as a yellow solid (215 mg, 96\% yield). ${ }^{1} \mathbf{H}$ NMR (400 $\left.\mathbf{~ M h z}, \mathbf{C D C l}_{3}\right) \delta$ 7.59-7.26 (m, phenyl-H), 6.69, $6.62(\mathrm{dd}, \mathrm{AB}$ coupling, $\mathrm{J}=8.8 \mathrm{~Hz}$ ), $6.10(\mathrm{t}, \mathrm{J}=7.1 \mathrm{~Hz}, \mathrm{C}=\mathrm{CH}), 5.39$ (s, residual $\left.\mathrm{HSiPh}_{3}\right), 3.66\left(\mathrm{~s}, 3 \mathrm{H}, \mathrm{O}-\mathrm{CH}_{3}\right), 1.99\left(\mathrm{q}, J=7.1 \mathrm{~Hz}, 2 \mathrm{H}, \mathrm{C}=\mathrm{CH}-\mathrm{CH}_{2}-\right), 1.18\left(\mathrm{~m},-\mathrm{CH}_{2}-\mathrm{CH}_{2}-\right), 0.72(\mathrm{t}, J=7.1$ $\left.\mathrm{Hz}, 3 \mathrm{H}, \mathrm{CH}_{2}-\mathrm{CH}_{3}\right) .{ }^{13} \mathrm{C}$ NMR $\left(100 \mathrm{Mhz}, \mathrm{CDCl}_{3}\right) \delta 157.6,148.7,137.9,136.5,135.8,134.5,129.7$, 129.2, 128.0, 127.7, 127.6, 113.3, 55.1, 31.6, 30.0, 22.4, 13.9. IR (film, cm ${ }^{-1}$ ): v 3067.96, 3048.17, 2955.28, 2919.84, 1427.98. HRMS (ESI) Calculated for $\mathrm{C}_{31} \mathrm{H}_{32} \mathrm{NaOSi}[\mathrm{M}+\mathrm{Na}]^{+} 471.2115$, measured 441.2115 .

Minor product: triphenyl-[(Z)-(1-(4-methoxyphenyl)-2-butylvinyl)]silane relevant signal: ${ }^{1} \mathbf{H}$ NMR $\left(400 \mathrm{MHz}, \mathrm{CDCl}_{3}\right) \delta 3.68\left(\mathrm{~s}, \mathrm{O}-\mathrm{CH}_{3}\right), 2.10\left(\mathrm{~m}, \mathrm{C}(\mathrm{Si})=\mathrm{CH}-\mathrm{CH}_{2}-\right)$. Other signals are overlapped with the major product.

Minor product: triphenyl-[(E)-1-butylvinyl-2-(4-methoxyphenyl))]silane relevant signal: ${ }^{\mathbf{1}} \mathbf{H} \mathbf{~ N M R}$ $\left(400 \mathrm{MHz}, \mathrm{CDCl}_{3}\right) \delta 7.14(\mathrm{~s}, \mathrm{CH}=\mathrm{C}), 3.72\left(\mathrm{~s}, \mathrm{O}-\mathrm{CH}_{3}\right), 2.36\left(\mathrm{~m}, \mathrm{CH}=\mathrm{C}(\mathrm{Si})-\mathrm{CH}_{2}-\right)$. Other signals are overlapped with the major product.

Minor product: triphenyl-[(Z)-1-butylvinyl-2-(4-methoxyphenyl))]silane relevant signal: ${ }^{1} \mathbf{H} \mathbf{~ N M R}$ (400 MHz, $\left.\mathrm{CDCl}_{3}\right) \delta 7.14(\mathrm{~s}, \mathrm{CH}=\mathrm{C}), 3.70\left(\mathrm{~s}, \mathrm{O}-\mathrm{CH}_{3}\right), 2.55\left(\mathrm{~m}, \mathrm{CH}=\mathrm{C}(\mathrm{Si})-\mathrm{CH}_{2^{-}}\right)$. Other signals are overlapped with the major product.

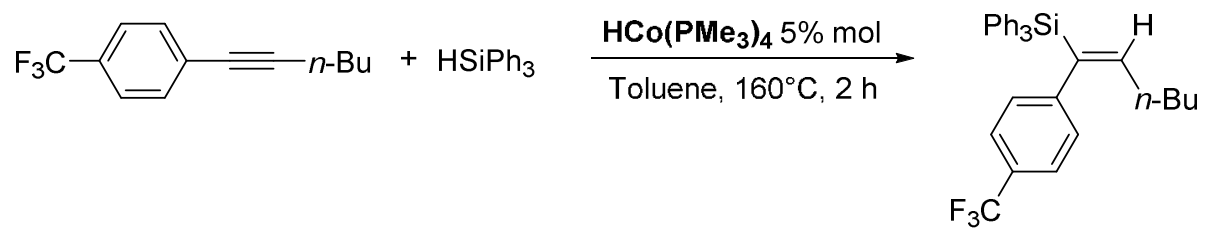

\section{Triphenyl-[(E)-(1-(4-(trifluoromethyl)phenyl)-2-butylvinyl)]silane (3da)}

The general procedure B was applied to 1-(4-(trifluoromethyl)phenyl)-1-hexyne 1d (113 mg, 0.5 $\mathrm{mmol}$ ) and triphenylsilane $(143 \mathrm{mg}, 0.55 \mathrm{mmol})$ in degassed toluene $(1 \mathrm{ml})$ then heated in an oil bath at $160{ }^{\circ} \mathrm{C}$ for $2 \mathrm{~h}$. Purification by silica gel flash-chromatography (pentane/ethyl acetate 99:1) 
afforded the title compound as a white solid (228 mg, 94\% yield). ${ }^{1} \mathbf{H} \mathbf{~ N M R ~ ( 4 0 0 ~} \mathbf{M H z}, \mathbf{C D C l}_{3}$ ) $\delta 7.39$ (m, phenyl-H, 18H), $6.97(\mathrm{~d}, J=8.0,2 \mathrm{H}), 6.30(\mathrm{t}, J=7.2 \mathrm{~Hz}, 1 \mathrm{H}), 2.06(\mathrm{q}, J=7.2 \mathrm{~Hz}, 2 \mathrm{H}$ ), $1.36(\mathrm{q}, J=$ $7.5 \mathrm{~Hz}, 2 \mathrm{H}), 1.28$ (q, $J=7.5 \mathrm{~Hz}, 2 \mathrm{H}), 0.85$ (t, $J=7.2 \mathrm{~Hz}, 3 \mathrm{H}) .{ }^{13} \mathrm{C} \mathrm{NMR}\left(100 \mathrm{MHz}, \mathrm{CDCl}_{3}\right) \delta 149.0$, $145.9,138.0,136.4,135.8$ (2C), 133.6, 129.5, 128.9, 127.7 (2C), 124.7, 124.7, 31.4, 30.2, 22.3, 13.8. IR (film, $\mathrm{cm}^{-1}$ ): v 3069.23, 3050.00, 2956.90, 2928.03, 212.80, 1323.38. HRMS (ESI) Calculated for $\mathrm{C}_{31} \mathrm{H}_{29} \mathrm{~F}_{3} \mathrm{LiSi}[\mathrm{M}+\mathrm{Li}]^{+}$493.2146, measured 493.2160.

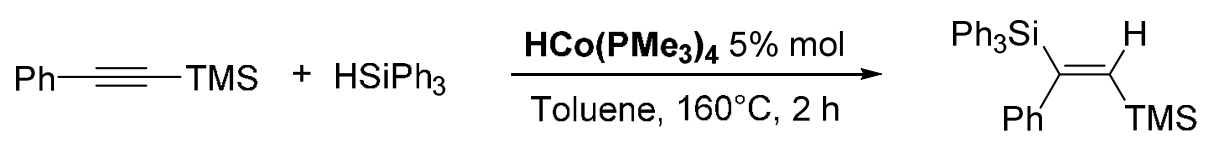

\section{Triphenyl-[(E)-(1-phenyl-2-(trimethylsilyl)vinyl)]silane (3ea)}

The general procedure B was applied to 1-phenyl-2-trimethylsilylacetylene 1e (87 mg, $0.5 \mathrm{mmol})$ and triphenylsilane $(143 \mathrm{mg}, 0.55 \mathrm{mmol})$ in degassed toluene $(1 \mathrm{ml})$ then heated in an oil bath at $160^{\circ} \mathrm{C}$ for $2 \mathrm{~h}$. Purification by silica gel flash-chromatography (pentane/ethyl acetate 99:1) afforded the title compound as a white solid (191 mg, 88\% yield). ${ }^{1} \mathrm{H}_{\mathrm{NMR}}\left(\mathbf{4 0 0} \mathbf{~ M h z}, \mathrm{CDCl}_{3}\right) \delta$ 7.58-7.44 (m, 15H), 7.28-7.26 (m, 3H), 7.03-7.01 (m, 2H), $6.79(\mathrm{~s}, 1 \mathrm{H}), 0.00(\mathrm{~s}, 9 \mathrm{H}) .{ }^{13} \mathrm{C}$ NMR (100 Mhz, $\left.\mathrm{CDCl}_{3}\right) \delta$ 159.5, 151.4, 144.6, 136.4 (2C), 134.0, 129.3, 128.1, 128.0, 127.6 (2C), 127.4, 125.9, 0.0. IR (film, $\mathrm{cm}^{-1}$ ): v 3068.47, 3049.82, 2352.35, 2360.48, 1420.63. HRMS (ESI) Calculated for $\mathrm{C}_{29} \mathrm{H}_{30} \mathrm{NaSi}_{2}$ $[\mathrm{M}+\mathrm{Na}]^{+}$457.1778, measured 457.1784 .
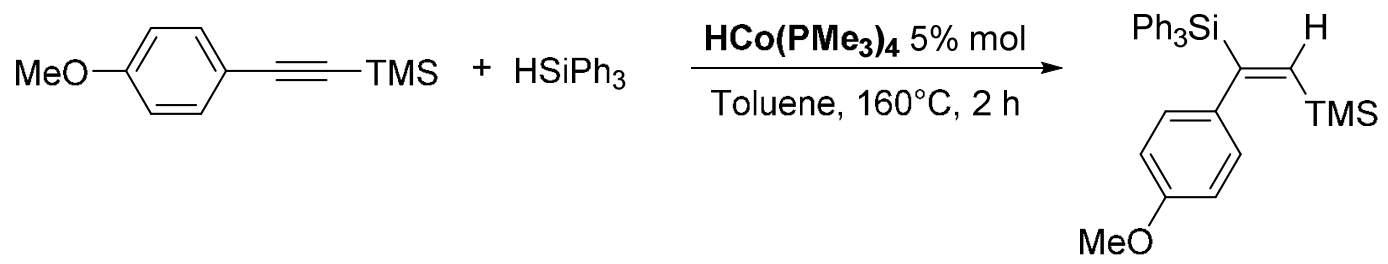

\section{Triphenyl-[(E)-(1-(4-methoxyphenyl)-2-(trimethylsilyl)vinyl)]silane (3fa)}

The general procedure B was applied to (4-methoxyphenylethynyl)trimethylsilane 1f (87 mg, 0.5 $\mathrm{mmol}$ ) and triphenylsilane $(102 \mathrm{mg}, 0.55 \mathrm{mmol})$ in degassed toluene $(1 \mathrm{ml})$ then heated in an oil bath at $160{ }^{\circ} \mathrm{C}$ for $2 \mathrm{~h}$. Purification by silica gel flash-chromatography (pentane/ethyl acetate 95:5) afforded the title compound as a white solid (200 mg, 86\% yield). ${ }^{1} \mathbf{H} \mathbf{~ N M R}\left(\mathbf{4 0 0} \mathbf{~ M h z}, \mathbf{C D C l}_{3}\right) \delta$ 7.56-7.41 (m, 15H), $6.91(\mathrm{~d}, J=8.7 \mathrm{~Hz}, 3 \mathrm{H}), 6.81(\mathrm{~d}, J=8.7 \mathrm{~Hz}, 3 \mathrm{H}), 6.75(\mathrm{~s}, 1 \mathrm{H}), 3.91(\mathrm{~s}, 3 \mathrm{H}), 0.00(\mathrm{~s}$, 9H). ${ }^{13} \mathrm{C}$ NMR (100 Mhz, $\left.\mathrm{CDCl}_{3}\right) \delta 159.0,157.9,151.6,136.4(2 \mathrm{C}), 134.1,129.2,129.1,127.5(2 \mathrm{C})$, 112.8, 55.0, 0.0. IR (film, $\mathrm{cm}^{-1}$ ): v 3133.73, 3045.49, 2125.40, 1503.43. HRMS (ESI) Calculated for $\mathrm{C}_{30} \mathrm{H}_{32} \mathrm{NaOSi}_{2}[\mathrm{M}+\mathrm{Na}]^{+}$487.1884, measured 487.1892. 

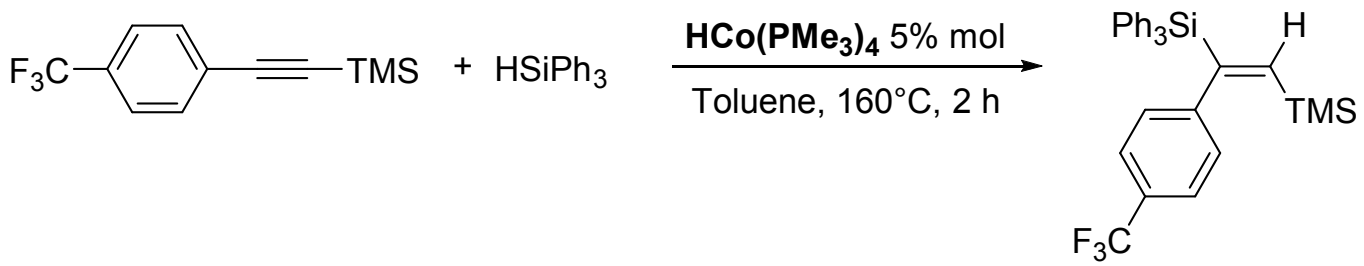

\section{Triphenyl-[(E)-(1-(4-trifluoromethyl)phenyl-2-(trimethylsilyl)vinyl)]silane (3ga)}

The general procedure B was applied to 1-((trimethylsilyl)ethynyl)-4-(trifluoromethyl)benzene $\mathbf{1 g}$ (121 $\mathrm{mg}, 0.5 \mathrm{mmol})$ and triphenylsilane $(102 \mathrm{mg}, 0.55 \mathrm{mmol})$ in degassed toluene $(1 \mathrm{ml})$ then heated in an oil bath at $160{ }^{\circ} \mathrm{C}$ for $2 \mathrm{~h}$. Purification by silica gel flash-chromatography (pentane/ethyl acetate 95:5) afforded the title compound as a yellow solid (203 mg, 81\% yield). ${ }^{1} \mathbf{H}$ NMR (400 Mhz, $\left.\mathrm{CDCl}_{3}\right) \delta 7.62-7.44(\mathrm{~m}, 17 \mathrm{H}), 7.09(\mathrm{~d}, J=8.0 \mathrm{~Hz}, 2 \mathrm{H}), 6.95(\mathrm{~s}, 1 \mathrm{H}), 0.00(\mathrm{~s}, 9 \mathrm{H}) .{ }^{13} \mathrm{C}$ NMR (100 Mhz, $\mathrm{CDCl}_{3}$ ) $\delta$ 158.5, 152.3, 136.4 (2C), 133.3, 129.6, 128.4, 127.8 (2C), 124.4, 0.0. IR (film, $\mathbf{~ c m}^{-1}$ ): v 3069.75, 2955.61, 2360.49, 2341.81, 1485.39. HRMS (ESI) Calculated for $\mathrm{C}_{30} \mathrm{H}_{29} \mathrm{~F}_{3} \mathrm{NaSi}_{2}[\mathrm{M}+\mathrm{Na}]^{+}$525.1652, measured 525.1636.
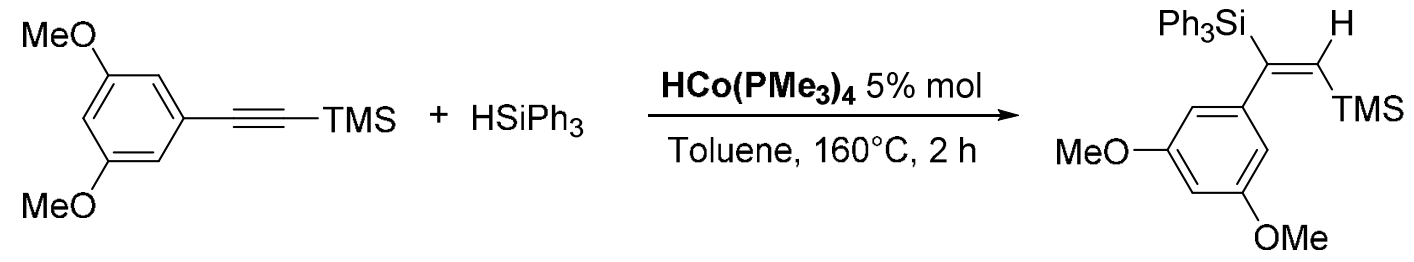

\section{Triphenyl-[(E)-(1-(3,5-dimethoxyphenyl)-2-(trimethylsilyl)vinyl)]silane (3ha)}

The general procedure B was applied to 1-[(trimethylsilyl)ethynyl]-3,5-dimethoxybenzene $\mathbf{1 h}$ (117 $\mathrm{mg}, 0.5 \mathrm{mmol})$ and triphenylsilane $(102 \mathrm{mg}, 0.55 \mathrm{mmol})$ in degassed toluene $(1 \mathrm{ml})$ then heated in an oil bath at $160{ }^{\circ} \mathrm{C}$ for $2 \mathrm{~h}$. Purification by silica gel flash-chromatography (pentane/ethyl acetate 95:5) afforded the title compound as a white solid ( $215 \mathrm{mg}, 87 \%$ yield). ${ }^{1} \mathbf{H} \mathbf{~ N M R}\left(\mathbf{4 0 0} \mathbf{~ M h z}, \mathbf{C D C l}_{3}\right.$ ) ठ 7.56-7.41 (m, 15H), $6.91(\mathrm{~d}, J=8.7 \mathrm{~Hz}, 3 \mathrm{H}), 6.81(\mathrm{~d}, J=8.7 \mathrm{~Hz}, 3 \mathrm{H}), 6.75(\mathrm{~s}, 1 \mathrm{H}), 3.91(\mathrm{~s}, 3 \mathrm{H}), 0.00$ (s, 9H). ${ }^{13}$ C NMR (100 Mhz, CDCl $_{3}$ ) $\delta 159.0,157.9,151.6,136.4$ (2C), 134.1, 129.2, 129.1, 127.5 (2C), 112.8, 55.0, 0.0. IR (film, cm ch $^{-1}$ v 3068.58, 2953.32, 23.59.98, 1588.10. HRMS (ESI) Calculated for $\mathrm{C}_{31} \mathrm{H}_{34} \mathrm{NaO}_{2} \mathrm{Si}_{2}[\mathrm{M}+\mathrm{Na}]^{+}$517.1990, measured 517.1998.

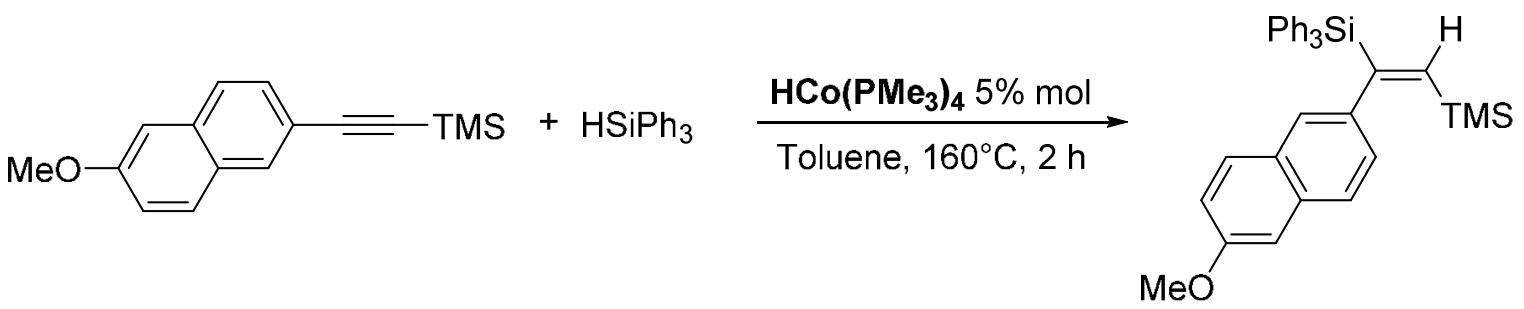




\section{Triphenyl-[(E)-(1-(6-methoxynaphthalen-2-yl)-2-(trimethylsilyl)vinyl)]silane (3ia)}

The general procedure B was applied to (6-methoxynaphthalen-2-ylethynyl)trimethylsilane 1i (127 $\mathrm{mg}, 0.5 \mathrm{mmol}$ ) and triphenylsilane $(102 \mathrm{mg}, 0.55 \mathrm{mmol})$ in degassed toluene $(1 \mathrm{ml})$ then heated in an oil bath at $160{ }^{\circ} \mathrm{C}$ for $2 \mathrm{~h}$. Purification by silica gel flash-chromatography (pentane/ethyl acetate 95:5) afforded the title compound as a white solid (193 mg, 75\% yield). ${ }^{1} \mathbf{H} \mathbf{~ N M R}$ (400 $\mathbf{~ M h z}, \mathbf{C D C l}_{3}$ ) $\delta 7.73-7.44(\mathrm{~m}, 18 \mathrm{H}), 7.30-7.25(\mathrm{~m}, 2 \mathrm{H}), 7.15(\mathrm{dd}, J=8.4,1.7 \mathrm{~Hz}, 1 \mathrm{H}), 6.90(\mathrm{~s}, 1 \mathrm{H}), 4.10(\mathrm{~s}, 3 \mathrm{H})$, 0.01 (s, 9H). ${ }^{13} \mathrm{C}$ NMR (100 Mhz, $\left.\mathrm{CDCl}_{3}\right) \delta 159.4,157.0,151.9,139.9,136.4,133.9,132.8,129.2$, 129.1, 128.3, 127.6, 127.5, 126.2, 125.5, 118.4, 105.4, 55.1, 0.0. IR (film, $\left.\mathbf{~ c m}^{-1}\right)$ : v 3067.88, 3050.32, 2952.89, 2360.41, 2341.30, 1601.69. HRMS (ESI) Calculated for $\mathrm{C}_{34} \mathrm{H}_{34} \mathrm{NaOSi}_{2}[\mathrm{M}+\mathrm{Na}]^{+} 537.2040$, measured 537.2042.
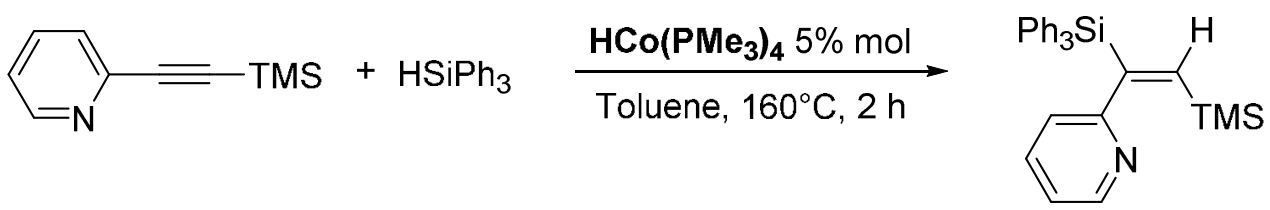

\section{Triphenyl-[(E)-(1-(2-pyridyl)-2-(trimethylsilyl)vinyl)]silane (3ja)}

The general procedure B was applied to 2-[(triethylsilyl)ethynyl]pyridine $\mathbf{1 j}(87 \mathrm{mg}, 0.5 \mathrm{mmol})$ and triphenylsilane $(102 \mathrm{mg}, 0.55 \mathrm{mmol})$ in degassed toluene $(1 \mathrm{ml})$ then heated in an oil bath at $160{ }^{\circ} \mathrm{C}$ for $2 \mathrm{~h}$. Purification by silica gel flash-chromatography (pentane/ethyl acetate $98: 2$ ) afforded the title compound as a white solid (200 mg, 92\% yield). ${ }^{1} \mathbf{H}$ NMR (400 Mhz, $\mathrm{CDCl}_{3}$ ) $\delta 8.60-8.57$ (m, $1 \mathrm{H}), 7.63-7.68(\mathrm{~m}, 16 \mathrm{H}), 7.15(\mathrm{~m}, 1 \mathrm{H}), 7.01(\mathrm{~d}, J=7.8 \mathrm{~Hz}, 1 \mathrm{H}), 6.87(\mathrm{~s}, 1 \mathrm{H}), 0.00(\mathrm{~s}, 9 \mathrm{H}) .{ }^{13} \mathrm{C}$ NMR (100 Mhz, $\mathrm{CDCl}_{3}$ ) $\delta 136.5$ (2C), 135.8, 134.1, 129.5, 128.9, 127.8 (2C), 127.6, 123.3, 121.2, 0.0. IR (film, $\mathbf{~ c m}^{-1}$ ): v 3068.47, 3048.73, 2896.32, 2360.65, 1427.43. HRMS (ESI) Calculated for $\mathrm{C}_{28} \mathrm{H}_{29} \mathrm{NNaSi}_{2}[\mathrm{M}+\mathrm{Na}]^{+}$458.1731, measured 458.1747.

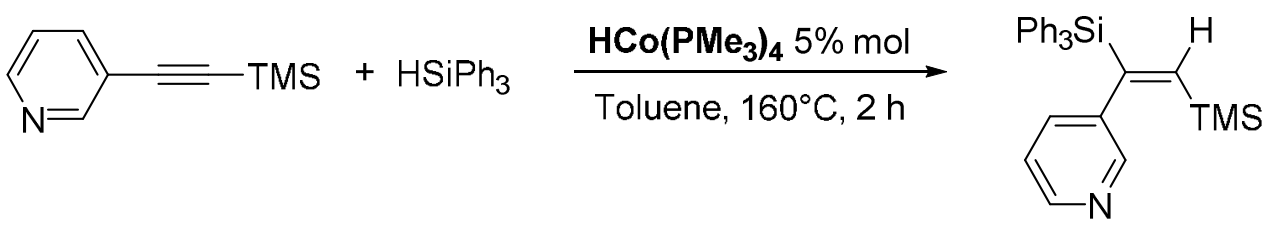

\section{Triphenyl-[(E)-(1-(3-pyridyl)-2-(trimethylsilyl)vinyl)]silane (3ka)}

The general procedure B was applied to 3-[(triethylsilyl)ethynyl]pyridine $1 \mathbf{k}(87 \mathrm{mg}, 0.5 \mathrm{mmol}$ ) and triphenylsilane $(102 \mathrm{mg}, 0.55 \mathrm{mmol})$ in degassed toluene $(1 \mathrm{ml})$ then heated in an oil bath at $160^{\circ} \mathrm{C}$ for $2 \mathrm{~h}$. Purification by silica gel flash-chromatography (pentane/ethyl acetate 98:2) afforded the title compound as a white solid (122 mg, 56\% yield). ${ }^{1} \mathbf{H}$ NMR (400 Mhz, $\left.\mathrm{CDCl}_{3}\right) \delta 7.57-7.38$ (m, $15 \mathrm{H}), 6.68(\mathrm{~s}, 1 \mathrm{H}), 6.35(\mathrm{t}, J=2.3 \mathrm{~Hz}, 1 \mathrm{H}), 6.10(\mathrm{~d}, J=2.3 \mathrm{~Hz}, 2 \mathrm{H}), 3.64(\mathrm{~s}, 6 \mathrm{H}), 0.00(\mathrm{~s}, 9 \mathrm{H}) .{ }^{13} \mathrm{C} \mathrm{NMR}$ (100 MHz, $\mathrm{CDCl}_{3}$ ) $\delta 159.7,159.1,150.9,146.4,136.4$ (2C), 133.8, 129.3, 127.5 (2C), 106.2, 98.7, 55.0, 0.0. IR (film, $\mathrm{cm}^{-1}$ ): v 3068.87, 3048.35, 2953.24, 2361.38, 1408.29. HRMS (ESI) Calculated for $\mathrm{C}_{28} \mathrm{H}_{30} \mathrm{NSi}_{2}[\mathrm{M}+\mathrm{H}]^{+}$436.1911, measured 436.1926. 

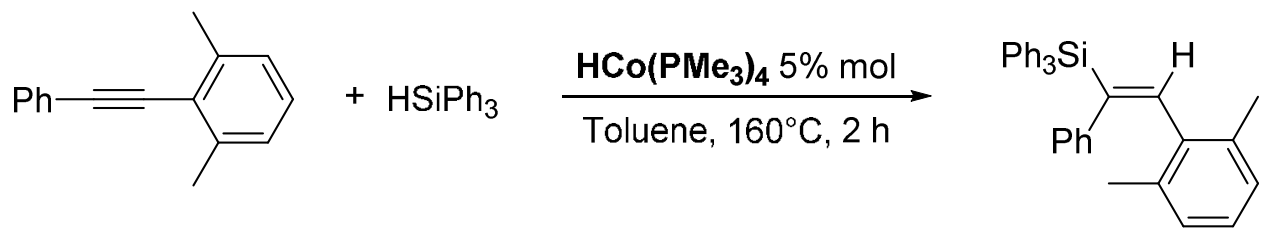

\section{Triphenyl-[(E)-(1-phenyl-2-(2,6-dimethylphenyl)-vinyl)]silane (3la)}

The general procedure B was applied to 1,3-dimethyl-2-(phenylethynyl)benzene ${ }^{9}$ 1l (103 mg, 0.5 $\mathrm{mmol}$ ) and triphenylsilane $(143 \mathrm{mg}, 0.55 \mathrm{mmol})$ in degassed toluene $(1 \mathrm{ml})$ then heated in an oil bath at $160{ }^{\circ} \mathrm{C}$ for $2 \mathrm{~h}$. Purification by silica gel flash-chromatography (pentane/ethyl acetate 99:1) afforded the title compound as a white solid (212 mg, 91\% yield). $\left.{ }^{1} \mathbf{H} \mathbf{~ N M R ~ ( 4 0 0 ~} \mathbf{M H z} \mathbf{C D C l}_{3}\right) \delta$ 7.56-7.24 (m, 6H), 7.42-7.33 (m, 9H), 7.00-6.94 (m, 4H), 6.87-6.80 (m, 4H), $2.13(\mathrm{~s}, 6 \mathrm{H}) .{ }^{13} \mathrm{C} \mathrm{NMR}$ $\left(100 \mathrm{MHz} \mathrm{CDCl}_{3}\right) \delta 145.4,141.7,141.3,137.6,136.5,135.2,134.4,129.5,128.2,127.8,127.6$, 127.0, 126.6, 126.0, 20.8. IR (film, $\mathrm{cm}^{-1}$ ): v 3059.36, 2909.57, 2363.56, 1378.26. HRMS (ESI) Calculated for $\mathrm{C}_{34} \mathrm{H}_{30} \mathrm{NaSi}[\mathrm{M}+\mathrm{Na}]^{+} 489.2009$, measured 489.1994 .

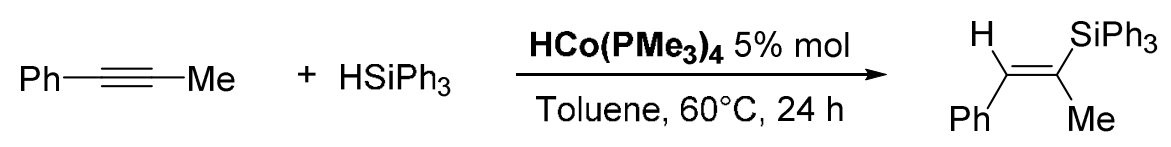

\section{Triphenyl-[(E)-(1-phenylprop-1-en-2-yl)]silane (3ma) ${ }^{10}$}

The general procedure $\mathrm{B}$ (heated at $60{ }^{\circ} \mathrm{C}$ for $24 \mathrm{~h}$ ) was applied to 1-phenyl-1-propyne $1 \mathrm{~m}(0.06$ $\mathrm{mL}, 0.5 \mathrm{mmol}$ ) and triphenylsilane $(102 \mathrm{mg}, 0.55 \mathrm{mmol})$ in degassed toluene $(1 \mathrm{ml})$ then heated in an oil bath at $60{ }^{\circ} \mathrm{C}$ for $24 \mathrm{~h}$. Purification by silica gel flash-chromatography (pentane/ethyl acetate 99:1) afforded the title compound as a white solid (35 mg, 19\% yield). ${ }^{1} \mathbf{H} \mathbf{N M R}\left(400 \mathrm{MHz}, \mathrm{CDCl}_{3}\right) \delta$ $7.68-7.64(\mathrm{~m}, 6 \mathrm{H}), 7.48-7.35(\mathrm{~m}, 14 \mathrm{H}), 6.96(\mathrm{q}, J=1.7 \mathrm{~Hz}, 1 \mathrm{H}), 2.14(\mathrm{~d}, J=1.8 \mathrm{~Hz}, 3 \mathrm{H}) .{ }^{13} \mathrm{C} \mathrm{NMR}$ (100 MHz, $\mathrm{CDCl}_{3}$ ) $\delta 143.6,138.1,136.3(2 \mathrm{C}), 135.7,134.4,134.0,129.5,129.1,128.0,127.8(2 \mathrm{C})$, 126.9, 18.2.
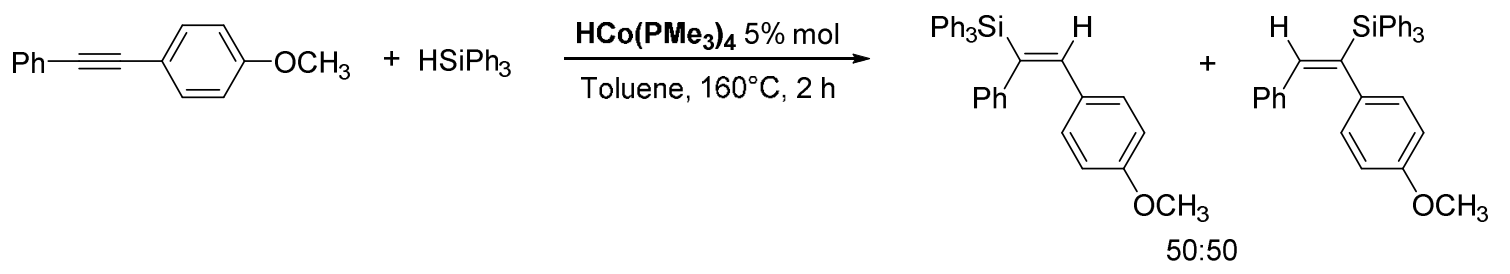
Triphenyl-[(E)-(1-(4-methoxyphenyl)-2-phenylvinyl)]silane and triphenyl-[(E)-(1-phenyl-2-(4methoxyphenyl)vinyl)]silane (3na)

The general procedure B was applied to 1-methoxy-4-(phenylethynyl)benzene 1n (104 mg,0.5 $\mathrm{mmol}$ ) and triphenylsilane $(143 \mathrm{mg}, 0.55 \mathrm{mmol})$ in degassed toluene $(1 \mathrm{ml})$ then heated in an oil bath at $160{ }^{\circ} \mathrm{C}$ for $2 \mathrm{~h}$. Purification by silica gel flash-chromatography (pentane/ethyl acetate 95:5) afforded a 1:1 mixture of the title compounds as a white solid (210 mg, 90\% yield). ${ }^{1} \mathbf{H}$ NMR (400 Mhz, $\left.\mathrm{CDCl}_{3}\right) \delta 7.40-6.80$ (m, aromatic- $H$ ), $7.36(\mathrm{~s}, 1 \mathrm{H}, \mathrm{C}=\mathrm{CH}), 6.72(\mathrm{~d}, J=8.7 \mathrm{~Hz}, 2 \mathrm{H}, \mathrm{AB}$ coupling), $6.61\left(\mathrm{~d}, J=8.8 \mathrm{~Hz}, 2 \mathrm{H}, \mathrm{AB}\right.$ coupling), $6.54\left(\mathrm{~d}, J=8.8 \mathrm{~Hz}, 2 \mathrm{H}, \mathrm{AB}\right.$ coupling), 3.67 (s, 3H, O- $\left.\mathrm{CH}_{3}\right), 3.64$ $\left(\mathrm{s}, 3 \mathrm{H}, \mathrm{O}-\mathrm{CH}_{3}\right) .{ }^{13} \mathrm{C} \mathrm{NMR}\left(100 \mathrm{Mhz}, \mathrm{CDCl}_{3}\right) \delta 158.9,157.9,143.6,142.7,142.1,140.6,138.2,137.30$, 136.6, 135.7, 134.0, 134.0, 131.1, 129.9, 129.7, 129.4, 129.4, 128.5, 128.4, 127.9, 127.7, 127.6, 125.8, 113.9, 113.3, 55.1, 55.0. IR (film, cm cm $^{-1}$ v 3067.78, 3048.84, 2360.03, 2341.08, 1603.25. HRMS (ESI) Calculated for $\mathrm{C}_{33} \mathrm{H}_{28} \mathrm{NaOSi}[\mathrm{M}+\mathrm{Na}]^{+}$491.1802, measured 491.1790.
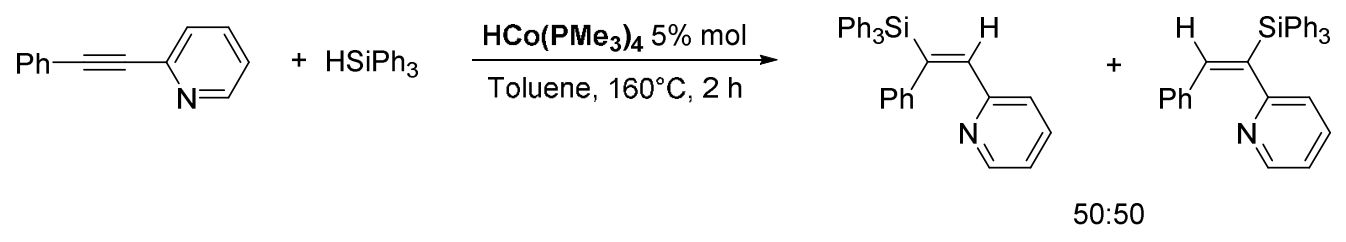

(E)-2-(2-phenyl-1-(triphenylsilyl)vinyl)pyridine and (E)-2-(2-phenyl-2-(triphenylsilyl)vinyl)pyridine (3oa)

The general procedure B was applied to 2-(phenylethynyl)pyridine 10 (90 $\mathrm{mg}, 0.5 \mathrm{mmol}$ ) and triphenylsilane $(143 \mathrm{mg}, 0.55 \mathrm{mmol})$ in degassed toluene $(1 \mathrm{ml})$ then heated in an oil bath at $160{ }^{\circ} \mathrm{C}$ for $2 \mathrm{~h}$. Purification by silica gel flash-chromatography (pentane/ethyl acetate 98:2) afforded a 1:1 mixture of the title compounds as a white solid (178 mg, 81\% yield). ${ }^{1} \mathbf{H}$ NMR (400 MHz, $\left.\mathrm{CDCl}_{3}\right) \delta$ 8.46 (ddd, $J=4.9,1.9,1.0 \mathrm{~Hz}, 2 \mathrm{H}$, pyridyl- $H), 7.58-7.54(\mathrm{~m}, 12 \mathrm{H}$, pyridyl- $H), 7.41-7.30(\mathrm{~m}, 21 \mathrm{H}$, phenyl-H), $7.13-7.09$ (m, 7H, phenyl-H), 6.99 (ddd, $J=7.5,4.9,1.2 \mathrm{~Hz}, 2 \mathrm{H}$, pyridyl-H), $6.94-6.90$ (m, 4H, pyridyl-H), 6.87 (s, $1 \mathrm{H}, \mathrm{CH}=\mathrm{CSi}), 6.85(\mathrm{~s}, 1 \mathrm{H}, \mathrm{CSi}=\mathrm{CH}) .{ }^{13} \mathrm{C} \mathbf{N M R}\left(100 \mathbf{~ M H z}, \mathbf{C D C l}_{3}\right) \delta 160.9$, $149.5,145.2,140.8,136.8,136.5,135.8,134.9,133.8,130.0,129.5,129.4,127.9,127.9,127.7$, 127.7, 123.7, 120.7. IR (film, $\mathrm{cm}^{-1}$ ): v 3057.78, 2969.87, 2855.68, 2363.82, 1589.85, 1427.64. HRMS (ESI) Calculated for $\mathrm{C}_{31} \mathrm{H}_{26} \mathrm{NSi}_{2}[\mathrm{M}+\mathrm{H}]^{+} 440.1829$, measured 440.1823 .

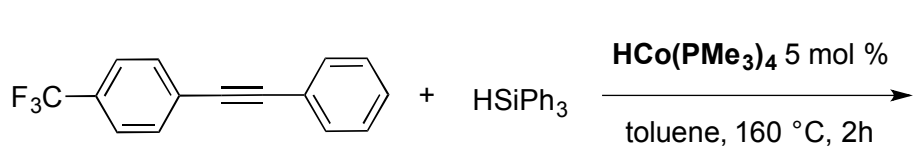

toluene, $160{ }^{\circ} \mathrm{C}, 2 \mathrm{~h}$

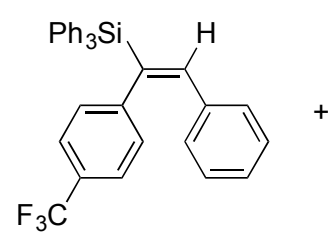

52/48 (major compound was not determined)

Triphenyl-[(E)-(1-(4-trifluoromethylphenyl)-2-phenylvinyl)]silane and triphenyl-[(E)-(1-phenyl-2(4-trifluoromethylphenyl)vinyl)]silane (3pa) 
The general procedure B was applied to 1-trifluoromethyl-4-(phenylethynyl)benzene 1n (123 mg, $0.5 \mathrm{mmol}$ ) and triphenylsilane $(143 \mathrm{mg}, 0.55 \mathrm{mmol})$ in degassed toluene $(1 \mathrm{ml})$ then heated in an oil bath at $160^{\circ} \mathrm{C}$ for $2 \mathrm{~h}$. Purification by silica gel flash-chromatography (pentane/ethyl acetate 95:5) afforded a 52:48 mixture of the title compounds as a yellow solid (203 mg, $80 \%$ yield). ${ }^{1} \mathbf{H}$ NMR $\left(400 \mathrm{MHz} \mathrm{CDCl}_{3}\right) \delta 7.48-7.32(\mathrm{~m}, 35 \mathrm{H}$, aromatic $\mathrm{C}-\mathrm{H}$, minor and Major), $7.16-7.09(\mathrm{~m}, 6 \mathrm{H}$, aromatic $\mathrm{C}-\mathrm{H}$, minor and Major), $7.16(\mathrm{~s}, 1 \mathrm{H}$, Major), 7.06-7.04 (d, 2H, J=8.1 Hz, minor), 7.03(s, $1 \mathrm{H}$, minor), $6.99-6.97$ (d, 2H, J=8.1 Hz, Major), $6.95-6.93$ (m, 2H, Major), $6.87-6.84(\mathrm{~m}, 2 \mathrm{H}$, minor). ${ }^{13} \mathrm{C}$ NMR $\left(100 \mathrm{MHz} \mathrm{CDCl}_{3}\right) \delta 146.0,144.5,144.0,141.6,141.2,140.5,139.9,136.5,136.5$, 135.2, 133.3, 133.1, 129.7, 129.7 (d, J = $3.1 \mathrm{~Hz}$ ), 128.8, 128.6, 128.1, 128.1, 127.8, 127.8, 127.7, 126.3, 125.3 (q, J = 3.7 Hz), 124.8 (q, J = 3.8 Hz). ${ }^{19} \mathrm{~F} \mathrm{NMR} \mathrm{(376} \mathrm{MHz,} \mathrm{CDCl} 3$ ) $\delta$-62.25 (Major), -62.63 (minor). IR (film, $\mathbf{c m}^{-1}$ ): v 3000, 1650, 1487, 1430, 1350, 1166, 837. HRMS (ESI) Calculated for $\mathrm{C}_{33} \mathrm{H}_{25} \mathrm{~F}_{3} \mathrm{NaSi}[\mathrm{M}+\mathrm{Na}]^{+}$529.6330, measured 529.6318 .

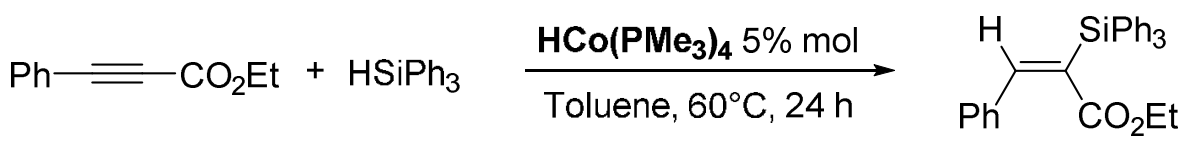

\section{(E)-Ethyl 3-phenyl-2-(triphenylsilyl)acrylate (3'qa) $)^{5}$}

The general procedure $B$ was applied to ethyl phenylpropiolate $1 p(0.08 \mathrm{~mL}, 0.5 \mathrm{mmol})$ and triphenylsilane $(102 \mathrm{mg}, 0.55 \mathrm{mmol})$ in degassed toluene $(1 \mathrm{ml})$ then heated in an oil bath at $60{ }^{\circ} \mathrm{C}$ for 24h. Purification by silica gel flash-chromatography (pentane/ethyl acetate 90:10) afforded the title compound as a white solid (35 mg, 19\% yield). ${ }^{1} \mathbf{H}$ NMR (400 MHz, $\left.\mathbf{C D C l}_{3}\right) \delta 7.70-7.65$ (m, $6 \mathrm{H}), 7.46-7.28(\mathrm{~m}, 14 \mathrm{H}), 6.95(\mathrm{~s}, 1 \mathrm{H}), 3.93(\mathrm{q}, J=7.1 \mathrm{~Hz}, 2 \mathrm{H}), 0.91(\mathrm{t}, J=7.1 \mathrm{~Hz}, 3 \mathrm{H}) .{ }^{13} \mathrm{C}$ NMR (100 $\left.\mathrm{MHz}, \mathrm{CDCl}_{3}\right) \delta 171.3,147.4,136.3(2 \mathrm{C}), 133.7,132.8,129.8,128.8,128.5,128.3,127.8(2 \mathrm{C}), 60.5$, 13.6.

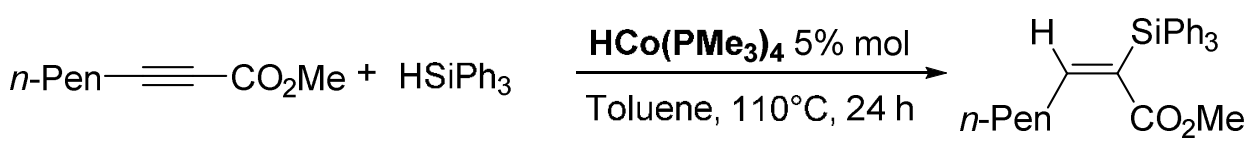

\section{(E)-Methyl 2-(triphenylsilyl)-2-octenoate (3'ra)}

The general procedure $B$ was applied to methyl 2-octynoate $1 q(0.08 \mathrm{~mL}, 0.5 \mathrm{mmol})$ and triphenylsilane $(102 \mathrm{mg}, 0.55 \mathrm{mmol})$ in degassed toluene $(1 \mathrm{ml})$ then heated in an oil bath at $110{ }^{\circ} \mathrm{C}$ for 24h. Purification by silica gel flash-chromatography (pentane/ethyl acetate 95:5) afforded the title compound as a white solid (85 mg, 50\% yield). ${ }^{1} \mathbf{H} \mathbf{~ N M R}\left(400 \mathbf{~ M H z}, \mathrm{CDCl}_{3}\right) \delta 7.60-7.54(\mathrm{~m}$, $6 \mathrm{H}), 7.43-7.34(\mathrm{~m}, 9 \mathrm{H}), 6.30(\mathrm{t}, J=7.3 \mathrm{~Hz}, 1 \mathrm{H}), 3.47(\mathrm{~s}, 3 \mathrm{H}), 2.45(\mathrm{q}, J=7.3 \mathrm{~Hz}, 2 \mathrm{H}), 1.41(\mathrm{t}, J=7.3$ $\mathrm{Hz}, 2 \mathrm{H}), 1.28(\mathrm{~m}, 4 \mathrm{H}),(\mathrm{t}, J=7.2 \mathrm{~Hz}, 3 \mathrm{H}) .{ }^{13} \mathrm{C}$ NMR (100 MHz, $\left.\mathbf{C D C l}_{3}\right) \delta 170.5,158.3,136.1,135.8$, 135.3, 128.0, 127.7, 51.0, 32.0, 31.4, 28.5, 22.3, 13.9. IR (film, $\left.\mathrm{cm}^{-1}\right):$ v 3060, 2969, 2921, 2360, 
2362, 2125, 17141428. HRMS (ESI) Calculated for $\mathrm{C}_{27} \mathrm{H}_{30} \mathrm{NaO}_{2} \mathrm{Si}[\mathrm{M}+\mathrm{Na}]^{+}$437.1907, measured 437.1899 .

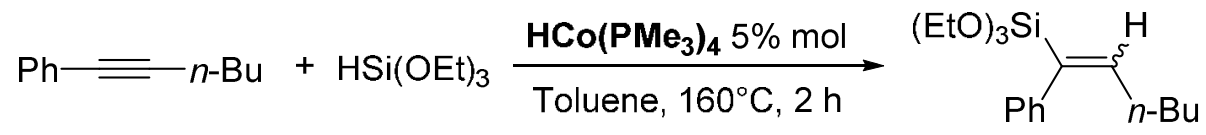

$83 / 17(E / Z)$

\section{Triethoxy-[(E)-(1-phenyl-2-butylvinyl)]silane (3bb) ${ }^{11}$}

The general procedure $C$ was applied to 1-phenyl-1-hexyne 1 b $(87 \mathrm{mg}, 0.5 \mathrm{mmol}$ ) and triethoxysilane $(0.1 \mathrm{~mL}, 0.55 \mathrm{mmol})$ in degassed toluene $(1 \mathrm{ml})$ then heated in an oil bath at $160{ }^{\circ} \mathrm{C}$ for $2 \mathrm{~h}$. Purification by silica gel flash-chromatography (pentane/ethyl acetate 95:5) afforded the title compound as a colorless oil (148 mg, 92\% yield). ${ }^{1} \mathbf{H}$ NMR (400 $\left.\mathbf{~ M h z , ~} \mathbf{C D C l}_{3}\right) \delta$ 7.23-7.03 (m, phenyl- $H$ ), 6.30 (t, $J=7.1 \mathrm{~Hz}, \mathrm{C}=\mathrm{CH}$ ), 3.70 (q, $\left.J=7 \mathrm{~Hz}, \mathrm{O}-\mathrm{CH}_{2}-\mathrm{CH}_{3}\right), 2.00$ (q, $J=7.2 \mathrm{~Hz}, 2 \mathrm{H}$ ), 1.19-1.10 $\left(\mathrm{m},-\mathrm{CH}_{2}-\mathrm{CH}_{2}-\right), 1.09$ (t, $\left.J=7 \mathrm{~Hz}, \mathrm{O}-\mathrm{CH}_{2}-\mathrm{CH}_{3}\right), 0.74(\mathrm{t}, J=7.1 \mathrm{~Hz}, 3 \mathrm{H}) .{ }^{13} \mathrm{C} \mathrm{NMR}(100 \mathrm{Mhz}, \mathrm{CDCl}) \delta$ $147.6,140.5,135.2,128.5,127.8,127.8,127.7,125.7,58.6,31.5,29.5,22.3,18.1,13.9$.

Minor product: triethoxy-[(Z)-1-phenyl-2-butylvinyl)]silane relevant signal: ${ }^{\mathbf{1}} \mathbf{H}$ NMR (400 $\mathbf{~ M h z ,}$ $\left.\mathrm{CDCl}_{3}\right) \delta 2.39(\mathrm{q}, J=7.4 \mathrm{~Hz})$. Other signals are overlapped with the $E$-isomer.
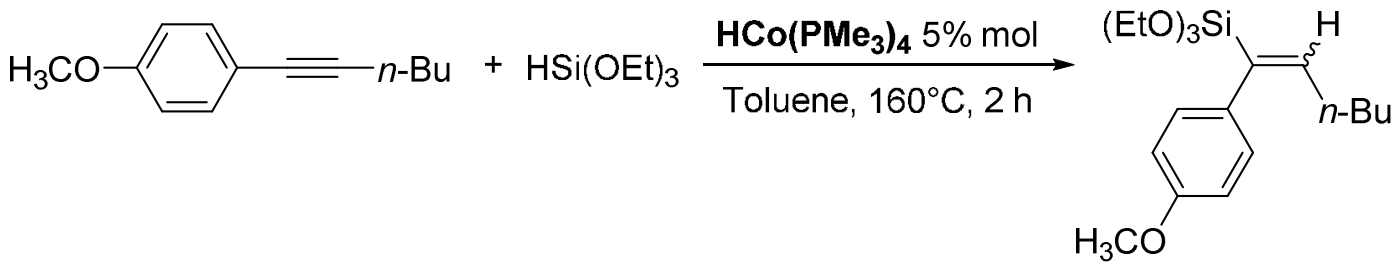

$82 / 18(E / Z)$

\section{Triethoxy-[(E)-(1-(4-methoxyphenyl)-2-butylvinyl)]silane (3cb)}

The general procedure $C$ was applied to 1-(4-methoxyphenyl)-1-hexyne 1c (94 mg, $0.5 \mathrm{mmol}$ ) and triethoxysilane $(0.1 \mathrm{~mL}, 0.55 \mathrm{mmol})$ in degassed toluene $(1 \mathrm{ml})$ then heated in an oil bath at $160{ }^{\circ} \mathrm{C}$ for $2 \mathrm{~h}$. Purification by silica gel flash-chromatography (pentane/ethyl acetate 95:5) afforded the title compound as a colorless oil (169 mg, 96\% yield). ${ }^{1} \mathbf{H}$ NMR (400 Mhz, $\left.\mathbf{C D C l}_{3}\right) \delta 6.99$ (d, $J=8.6$, $2 \mathrm{H}), 6.76(\mathrm{~d}, J=8.6,2 \mathrm{H}), 6.27(\mathrm{t}, J=7.1 \mathrm{~Hz}, \mathrm{C}=\mathrm{CH}), 3.71\left(\mathrm{~m}, \mathrm{O}-\mathrm{CH}_{2}-\mathrm{CH}_{3}\right.$ and $\left.\mathrm{O}-\mathrm{CH}_{3}\right), 2.02(\mathrm{q}, J=7.4$ $\mathrm{Hz}, 2 \mathrm{H}), 1.26-1.16\left(\mathrm{~m},-\mathrm{CH}_{2}-\mathrm{CH}_{2}-\right), 1.09\left(\mathrm{t}, J=7 \mathrm{~Hz}, \mathrm{O}-\mathrm{CH}_{2}-\mathrm{CH}_{3}\right), 0.75(\mathrm{t}, J=7.1 \mathrm{~Hz}, 3 \mathrm{H}) .{ }^{13} \mathrm{C}$ NMR (100 $\left.\mathrm{Mhz}, \mathrm{CDCl}_{3}\right) \delta 157.7,147.4,134.4,132.6,129.5,113.3,58.6,55.1,31.6,29.4,22.3,18.1,13.9$. IR (film, $\mathbf{c m}^{-1}$ ): v 2979.15, 2925.73, 2360.78, 2341.41, 1508.31. HRMS (ESI) Calculated for $\mathrm{C}_{19} \mathrm{H}_{32} \mathrm{NaO}_{4} \mathrm{Si}[\mathrm{M}+\mathrm{Na}]^{+}$375.1962, measured 375.1957.

Minor product: triethoxy-[(Z)-1-(4-methoxyphenyl)-2-butylvinyl)]silane relevant signal: ${ }^{\mathbf{1}} \mathbf{H} \mathbf{~ N M R}$ $\left(400 \mathrm{Mhz}, \mathrm{CDCl}_{3}\right) \delta 2.37(\mathrm{q}, J=7.4 \mathrm{~Hz})$. Other signals are overlapped with the $E$-isomer. 

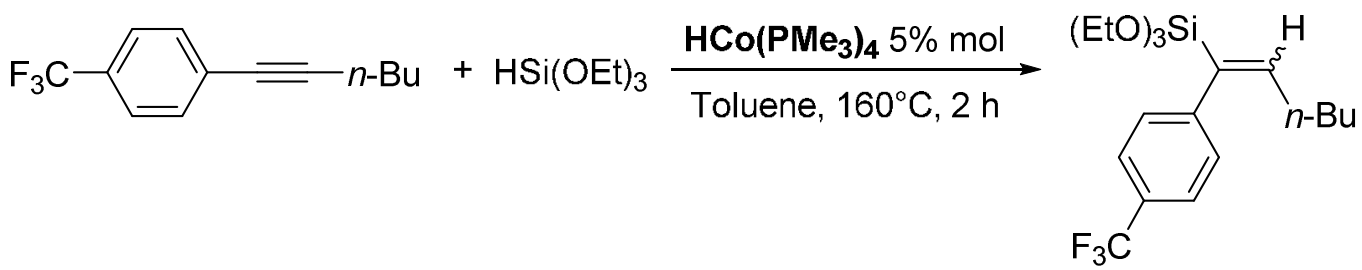

$77 / 23(E / Z)$

\section{Triethoxy-[(E)-(1-(4-(trifluoromethyl)phenyl)-2-butylvinyl)]silane (3db)}

The general procedure $C$ was applied to 1-(4-(trifluoromethyl)phenyl)-1-hexyne $1 \mathbf{d}$ (113 mg, 0.5 mmol) and triethoxysilane $(0.1 \mathrm{~mL}, 0.55 \mathrm{mmol})$ in degassed toluene $(1 \mathrm{ml})$ then heated in an oil bath at $160^{\circ} \mathrm{C}$ for $2 \mathrm{~h}$. Purification by silica gel flash-chromatography (pentane/ethyl acetate 95:5) afforded the title compound as a colorless oil (183 mg, 96\% yield). ${ }^{1} \mathbf{H}$ NMR (400 $\mathbf{~ M h z}, \mathbf{C D C l}_{3}$ ) $\delta$ $7.48(\mathrm{~d}, J=8.3,2 \mathrm{H}), 7.17(\mathrm{~d}, J=8.4,2 \mathrm{H}), 6.34(\mathrm{t}, J=7.2 \mathrm{~Hz}, \mathrm{C}=\mathrm{CH}), 3.71\left(\mathrm{q}, J=7.0 \mathrm{~Hz}, \mathrm{O}-\mathrm{CH}_{2}-\mathrm{CH}_{3}\right)$, 1.96 (q, J= 7.2 Hz, 2H), 1.20-1.16 (m, $-\mathrm{CH}_{2}-\mathrm{CH}_{2}-$ ), 1.10 (t, $\left.J=7 \mathrm{~Hz}, \mathrm{O}-\mathrm{CH}_{2}-\mathrm{CH}_{3}\right), 0.75$ (t, J= 7.2 Hz, 3H). ${ }^{13} \mathrm{C}$ NMR (100 Mhz, $\left.\mathrm{CDCl}_{3}\right) \delta 153.7,148.4,134.5,128.7,128.0,124.8,58.7,31.4,29.5,22.3$, 18.0, 13.8. IR (film, cm ${ }^{-1}$ ): v 2974.54, 2928.31, 2360.34, 2341.54, 1614.72. HRMS (ESI) Calculated for $\mathrm{C}_{19} \mathrm{H}_{29} \mathrm{~F}_{3} \mathrm{NaO}_{3} \mathrm{Si}[\mathrm{M}+\mathrm{Na}]^{+}$413.1730, measured 413.1714.

Minor product: triethoxy-[(Z)-(1-(4-(trifluoromethyl)phenyl)-2-butylvinyl)]silane relevant signal: ${ }^{1} \mathbf{H}$ NMR $\left(400 \mathrm{Mhz}, \mathrm{CDCl}_{3}\right) \delta 2.40(\mathrm{q}, J=7.2 \mathrm{~Hz})$. Other signals are overlapped with the $E$-isomer.

$$
\mathrm{Ph}=\mathrm{TMS}+\mathrm{HSi}(\mathrm{OEt})_{3} \frac{\mathrm{HCo}\left(\mathrm{PMe}_{3}\right)_{4} 5 \% \mathrm{~mol}}{\mathrm{Toluene}_{1} 160^{\circ} \mathrm{C}, 2 \mathrm{~h}} \underset{\mathrm{Ph}}{(\mathrm{EtO})_{3} \mathrm{Si}}
$$

$63 / 37(E / Z)$

\section{Triethoxy-[(E)-(1-phenyl-2-(trimethylsilyl)vinyl)]silane (3eb) $)^{12}$}

The general procedure $C$ was applied to 1-phenyl-2-trimethylsilylacetylene 1e (87 $\mathrm{mg}, 0.5 \mathrm{mmol})$ and triethoxysilane $(0.1 \mathrm{~mL}, 0.55 \mathrm{mmol})$ in degassed toluene $(1 \mathrm{ml})$ then heated in an oil bath at $160{ }^{\circ} \mathrm{C}$ for $2 \mathrm{~h}$. Purification by silica gel flash-chromatography (pentane/ethyl acetate $95: 5$ ) afforded the title compound as a colorless oil (160 mg, 95\% yield). ${ }^{1} \mathbf{H}$ NMR (400 Mhz, $\left.\mathbf{C D C l}_{3}\right)$ \% 7.42-7.26 (m, phenyl-H), 6.85 (s, C=CH), 3.92 (q, J = $7 \mathrm{~Hz}, \mathrm{O}-\mathrm{CH}_{2}-\mathrm{CH}_{3}$ ), 1.32 (t, J= $\left.7 \mathrm{~Hz}, \mathrm{O}-\mathrm{CH}_{2}-\mathrm{CH}_{3}\right), 0.00$ (s, TMS). ${ }^{13} \mathrm{C}$ NMR (100 Mhz, $\left.\mathrm{CDCl}_{3}\right) \delta 156.2,154.6,150.5,128.5,127.9,127.8,127.6,126.4,58.4,18.1$, 00.0 .

Minor product: triethoxy-[(Z)-(1-phenyl-2-(trimethylsilyl)vinyl)]silane relevant signals: ${ }^{1} \mathbf{H}$ NMR (400 Mhz, $\left.\mathrm{CDCl}_{3}\right) \delta 6.94$ (s, C=CH), 0.36 (s, TMS). ${ }^{13} \mathrm{C}$ NMR (100 Mhz, $\left.\mathrm{CDCl}_{3}\right) \delta 57.9\left(\mathrm{O}-\mathrm{CH}_{2}-\mathrm{CH}_{3}\right), 0.4$ (TMS). Other signals are overlapped with the $E$-isomer. 

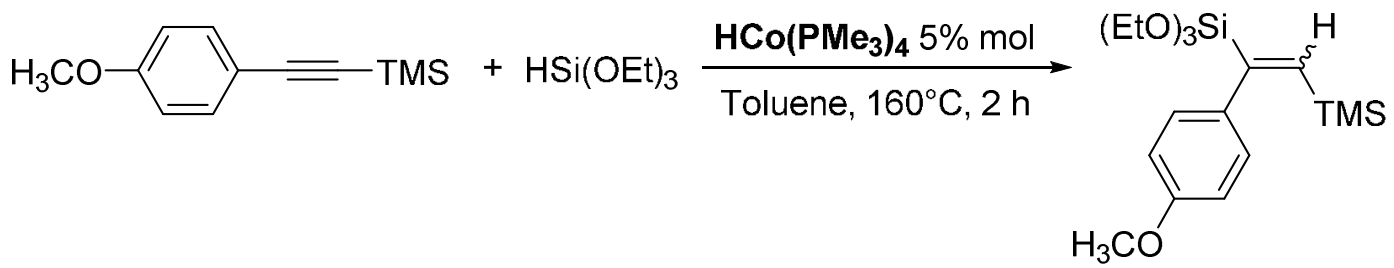

$72 / 28(E / Z)$

Triethoxy-[(E)-(1-(4-methoxyphenyl)-2-(trimethylsilyl)vinyl)]silane (3fb)

The general procedure $C$ was applied to (4-methoxyphenylethynyl)trimethylsilane 1 f $(87 \mathrm{mg}, 0.5$ mmol) and triethoxysilane $(0.1 \mathrm{~mL}, 0.55 \mathrm{mmol})$ in degassed toluene $(1 \mathrm{ml})$ then heated in an oil bath at $160{ }^{\circ} \mathrm{C}$ for $2 \mathrm{~h}$. Purification by silica gel flash-chromatography (pentane/ethyl acetate 95:5) afforded the title compound as a yellow oil (175 mg, 95\% yield). ${ }^{1} \mathbf{H} \mathbf{~ N M R ~ ( 4 0 0 ~} \mathbf{~ M h z}, \mathrm{CDCl}_{3}$ ) $\delta 7.42-$ $6.94\left(\mathrm{dd}, J=8.6,3.6 \mathrm{~Hz}, \mathrm{AB}\right.$ coupling), $6.88(\mathrm{~s}, \mathrm{C}=\mathrm{CH}), 3.91\left(\mathrm{~m}, \mathrm{O}-\mathrm{CH}_{2}-\mathrm{CH}_{3}\right.$ and $\left.\mathrm{O}-\mathrm{CH}_{3}\right), 1.30$ (t, $J=7$ $\left.\mathrm{Hz}, \mathrm{O}-\mathrm{CH}_{2}-\mathrm{CH}_{3}\right), 0.33(\mathrm{~s}, 9 \mathrm{H}) .{ }^{13} \mathrm{C}$ NMR $\left(100 \mathrm{Mhz}, \mathrm{CDCl}_{3}\right) \delta 158.2,150.2,136.2,130.2,128.7,112.9$, 58.6, 18.0, 0.0. IR (film, $\mathrm{cm}^{-1}$ ): v 2973.43, 2836.35, 2360.38, 1506.23. HRMS (ESI) Calculated for $\mathrm{C}_{18} \mathrm{H}_{32} \mathrm{NaO}_{4} \mathrm{Si}_{2}[\mathrm{M}+\mathrm{Na}]^{+}$391.1731, measured 391.1743.

Minor product: Triethoxy-[(Z)-(1-(4-methoxyphenyl)-2-(trimethylsilyl)vinyl)]silane relevant signals: ${ }^{1} \mathrm{H}$ NMR (400 Mhz, $\left.\mathrm{CDCl}_{3}\right) \delta 7.18,6.94$ (dd, $J=8.2,3.6 \mathrm{~Hz}, \mathrm{AB}$ coupling), 6.79 (s, C=CH), 0.37 (s, TMS). ${ }^{13} \mathrm{C}$ NMR (100 Mhz, $\left.\mathrm{CDCl}_{3}\right) \delta 58.3\left(\mathrm{O}-\mathrm{CH}_{2}-\mathrm{CH}_{3}\right), 17.9\left(\mathrm{O}-\mathrm{CH}_{2}-\mathrm{CH}_{3}\right), 0.4$ (TMS). Other signals are overlapped with the $E$-isomer.
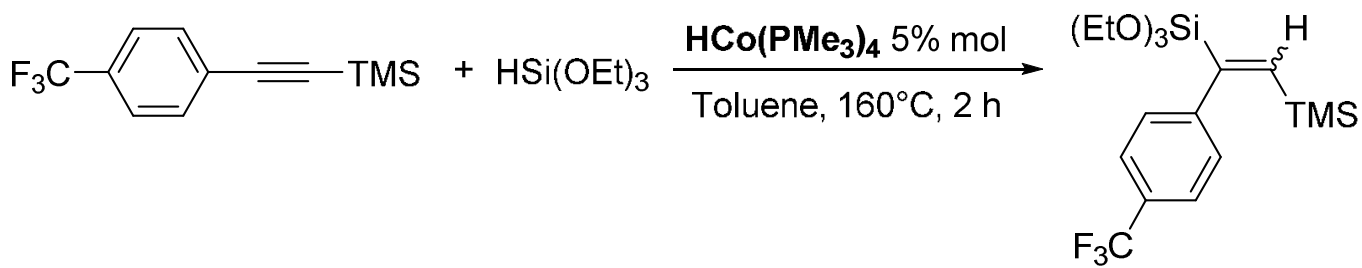

$52 / 48(E / Z)$

\section{Triethoxy-[(E)-(1-(4-trifluoromethyl)phenyl-2-(trimethylsilyl)vinyl)]silane (3gb)}

The general procedure $C$ was applied to 1-((trimethylsilyl)ethynyl)-4-(trifluoromethyl)benzene $\mathbf{1 g}$ (121 $\mathrm{mg}, 0.5 \mathrm{mmol})$ and triethoxysilane $(0.1 \mathrm{~mL}, 0.55 \mathrm{mmol})$ in degassed toluene $(1 \mathrm{ml})$ then heated in an oil bath at $160{ }^{\circ} \mathrm{C}$ for $2 \mathrm{~h}$. Purification by silica gel flash-chromatography (pentane/ethyl acetate 95:5) afforded the title compound as a yellow solid (183 mg, 95\% yield). ${ }^{1} \mathbf{H}$ NMR (400 MHz, $\mathrm{CDCl}_{3}$ ) $\delta 7.68,7.40$ (dd, $J=8.3 \mathrm{~Hz}, \mathrm{AB}$ coupling), 6.95 (s, C=CH), 3.94 (q, $J=7 \mathrm{~Hz}, \mathrm{O}$ $\left.\mathrm{CH}_{2}-\mathrm{CH}_{3}\right), 1.32$ (t, $\left.J=7 \mathrm{~Hz}, \mathrm{O}-\mathrm{CH}_{2}-\mathrm{CH}_{3}\right), 0.00$ (s, TMS). $\left.{ }^{13} \mathrm{C} \mathrm{NMR} \mathrm{(100} \mathrm{MHz,} \mathrm{CDCl}_{3}\right) \delta 156.7,155.2$, 150.9, 128.1, 125.8, 124.9, 124.6, 58.9, 18.1, 00.0. IR (film, $\mathrm{cm}^{-1}$ ): v 2975.52, 2928.96, 2358.84, 2340.47, 1614.16. HRMS (ESI) Calculated for $\mathrm{C}_{18} \mathrm{H}_{29} \mathrm{~F}_{3} \mathrm{NaO}_{3} \mathrm{Si}_{2}[\mathrm{M}+\mathrm{Na}]^{+} 429.1500$, measured 429.1498 .

Minor product: Triethoxy-[(Z)-(1-(4-trifluoromethyl)phenyl-2-(trimethylsilyl)vinyl)]silane relevant signals: ${ }^{1} \mathrm{H}$ NMR (400 MHz, $\mathrm{CDCl}_{3}$ ) $\delta 7.68,7.57$ (dd, $J=8.2 \mathrm{~Hz}, \mathrm{AB}$ coupling), 6.91 (s, C=CH), 0.37 (s, 
TMS). ${ }^{13} \mathrm{C} \mathrm{NMR}\left(100 \mathrm{MHz}, \mathrm{CDCl}_{3}\right) \delta 58.6\left(\mathrm{O}-\mathrm{CH}_{2}-\mathrm{CH}_{3}\right), 18.0\left(\mathrm{O}-\mathrm{CH}_{2}-\mathrm{CH}_{3}\right), 0.3$ (TMS). Other signals are overlapped with the $E$-isomer.

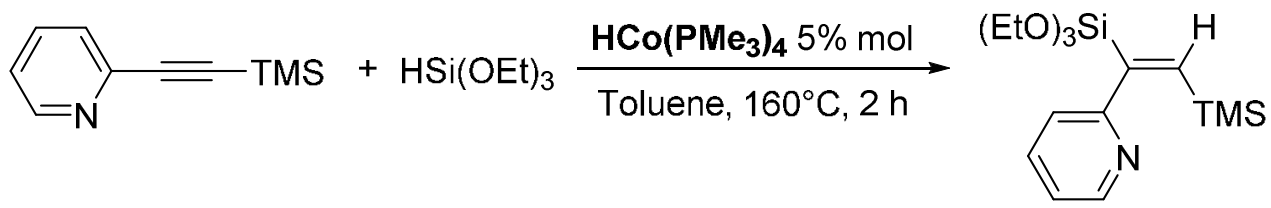

$100 / 0(E / Z)$

\section{Triethoxy-[(E)-(1-(2-pyridyl)-2-(trimethylsilyl)vinyl)]silane (3jb)}

The general procedure $C$ was applied to 2 -[(triethylsilyl)ethynyl]pyridine $1 \mathbf{j}$ ( $87 \mathrm{mg}, 0.5 \mathrm{mmol}$ ) and triethoxysilane $(0.1 \mathrm{~mL}, 0.55 \mathrm{mmol})$ in degassed toluene $(1 \mathrm{ml})$ then heated in an oil bath at $160{ }^{\circ} \mathrm{C}$ for $2 \mathrm{~h}$. Purification by silica gel flash-chromatography (pentane/ethyl acetate 90:10) afforded the title compound as a yellow oil (51 mg, 30\% yield). $\left.{ }^{1} \mathbf{H} \mathbf{~ N M R ~ ( 4 0 0 ~} \mathbf{~ M h z}, \mathbf{C D C l}_{3}\right) \delta 8.58-8.48(\mathrm{~m}, 1 \mathrm{H})$, $7.59(\mathrm{~m}, 1 \mathrm{H}), 7.36(\mathrm{~d}, J=7.9 \mathrm{~Hz}, 1 \mathrm{H}), 7.10(\mathrm{~m}, 1 \mathrm{H}), 6.88(\mathrm{~s}, 1 \mathrm{H}, \mathrm{C}=\mathrm{CH}), 3.82(\mathrm{q}, J=7.0 \mathrm{~Hz}, 6 \mathrm{H}), 1.18$ $(\mathrm{t}, J=7.0 \mathrm{~Hz}, 9 \mathrm{H}),-0.05(\mathrm{~s}, 9 \mathrm{H}) .{ }^{13} \mathrm{C}$ NMR $\left(100 \mathrm{Mhz}, \mathrm{CDCl}_{3}\right) \delta 160.1,154.5,153.1,148.2,135.3$, 122.6, 121.1, 58.5, 17.8, 0.0. IR (film, $\mathrm{cm}^{-1}$ ): v 3030.27, 2952.78, 2897.21, 1076.65. HRMS (ESI) Calculated for $\mathrm{C}_{16} \mathrm{H}_{30} \mathrm{NO}_{3} \mathrm{Si}_{2}[\mathrm{M}+\mathrm{H}]^{+} 340.1759$, measured 340.1748 .

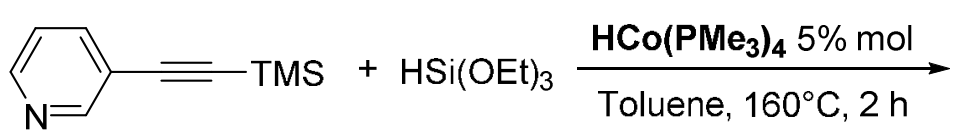<smiles>CCO[Si]C(=CC(C)(C)C)c1cccnc1</smiles>

$100 / 0(E / Z)$

\section{Triethoxy-[(E)-(1-(3-pyridyl)-2-(trimethylsilyl)vinyl)]silane (3kb)}

The general procedure $C$ was applied to 3-[(triethylsilyl)ethynyl]pyridine $1 \mathbf{k}(87 \mathrm{mg}, 0.5 \mathrm{mmol}$ ) and triethoxysilane $(0.1 \mathrm{~mL}, 0.55 \mathrm{mmol})$ in degassed toluene $(1 \mathrm{ml})$ then heated in an oil bath at $160{ }^{\circ} \mathrm{C}$ for $2 \mathrm{~h}$. Purification by silica gel flash-chromatography (pentane/ethyl acetate 90:10) afforded the title compound as a yellow oil (76 mg, $45 \%$ yield). ${ }^{1} \mathrm{H}$ NMR (400 MHz, $\left.\mathrm{CDCl}_{3}\right) \delta 8.47$ (bs, 2H), $7.51-$ $7.43(\mathrm{~m}, 1 \mathrm{H}), 7.21(\mathrm{~s}, 1 \mathrm{H}), 6.80(\mathrm{~s}, 1 \mathrm{H}, \mathrm{C}=\mathrm{CH}), 3.78(\mathrm{q}, J=7.0 \mathrm{~Hz}, 7 \mathrm{H}), 1.17(\mathrm{t}, J=7.0 \mathrm{~Hz}, 1 \mathrm{H}),-0.14$ (s, 9H). ${ }^{13} \mathrm{C}$ NMR (100 MHz, $\left.\mathrm{CDCl}_{3}\right) \delta 157.1,152.8(2 \mathrm{C}), 152.3,148.5,147.6,135.0,58.8,18.1,0.0$. IR (film, cm ${ }^{-1}$ ): v 3028.02, 2972.19, 2360.54, 1077.38. HRMS (ESI) Calculated for $\mathrm{C}_{16} \mathrm{H}_{30} \mathrm{NO}_{3} \mathrm{Si}_{2}$ $[\mathrm{M}+\mathrm{H}]^{+} 340.1759$, measured 340.1754 . 

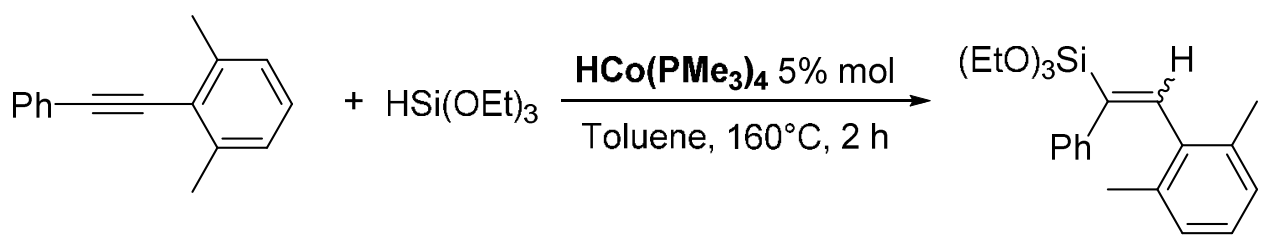

$100 / 0(E / Z)$

\section{Triethoxy-[(E)-(1-phenyl-2-(2,6-dimethylphenyl)vinyl)]silane (3lb)}

The general procedure C was applied to 1,3-dimethyl-2-(phenylethynyl)benzene 1 l (103 mg, 0.5 $\mathrm{mmol}$ ) and triethoxysilane $(0.1 \mathrm{~mL}, 0.55 \mathrm{mmol})$ in degassed toluene $(1 \mathrm{ml})$ then heated in an oil bath at $160^{\circ} \mathrm{C}$ for $2 \mathrm{~h}$. Purification by silica gel flash-chromatography (pentane/ethyl acetate 95:5) afforded the title compound as a colorless oil (130 mg, 70\% yield). $\left.{ }^{1} \mathbf{H} \mathbf{~ N M R ~ ( 4 0 0 ~} \mathbf{M H z} \mathbf{C D C l}_{3}\right) \delta$ $7.28(\mathrm{~s}, 1 \mathrm{H}), 7.09-6.90(\mathrm{~m}, 8 \mathrm{H}), 3.89(\mathrm{q}, J=7.0 \mathrm{~Hz}, 6 \mathrm{H}), 2.09(\mathrm{~s}, 6 \mathrm{H}), 1.23(\mathrm{t}, J=7 \mathrm{~Hz}, 9 \mathrm{H}) .{ }^{13} \mathrm{C} \mathrm{NMR}$ $\left(100 \mathrm{MHz} \mathrm{CDCl}_{3}\right) \delta 143.0,140.2,137.9,137.2,135.1,128.1,127.6,127.6,127.1,126.7,126.1$, 77.3, 77.0, 76.6, 58.7, 20.4, 18.1. IR (film, $\mathrm{cm}^{-1}$ ): v 2974.25, 2925.92, 2365.52, 1596.86. HRMS (ESI) Calculated for $\mathrm{C}_{22} \mathrm{H}_{30} \mathrm{NaO}_{3} \mathrm{Si}[\mathrm{M}+\mathrm{Na}]^{+} 393.1856$, measured 193.1855 .

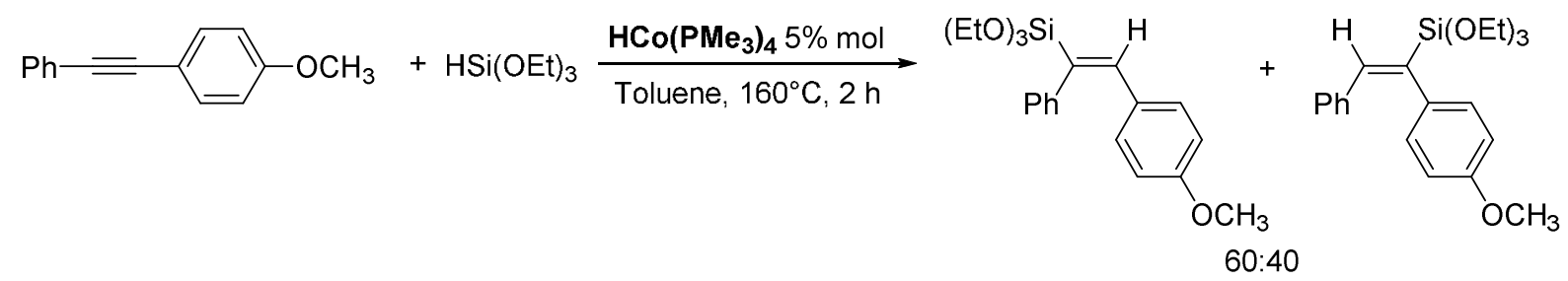

Triethoxy-[(E)-(1-phenyl-2-(4-methoxyphenyl)vinyl)]silane (3nb)

The general procedure $C$ was applied to 1-methoxy-4-(phenylethynyl)benzene 1n (104 mg,0.5 $\mathrm{mmol})$ and triethoxysilane $(0.1 \mathrm{~mL}, 0.55 \mathrm{mmol})$ in degassed toluene $(1 \mathrm{ml})$ then heated in an oil bath at $160{ }^{\circ} \mathrm{C}$ for $2 \mathrm{~h}$. Purification by silica gel flash-chromatography (pentane/ethyl acetate 90:10) afforded the title compound as a white solid (177 mg, 96\% yield). $\left.{ }^{1} \mathbf{H} \mathbf{~ N M R ~ ( 4 0 0 ~} \mathbf{M h z}, \mathrm{CDCl}_{3}\right) \delta$ 7.21-7.00 (m, aromatic- $H), 6.56(\mathrm{~d}, J=8.8,2 \mathrm{H}), 3.75\left(\mathrm{q}, J=7.1 \mathrm{~Hz}, \mathrm{O}-\mathrm{CH}_{2}-\mathrm{CH}_{3}\right), 3.64\left(\mathrm{~s}, 3 \mathrm{H}, \mathrm{O}-\mathrm{CH}_{3}\right)$, $1.02\left(\mathrm{t}, J=7 \mathrm{~Hz}, \mathrm{O}-\mathrm{CH}_{2}-\mathrm{CH}_{3}\right) .{ }^{13} \mathrm{C}$ NMR $\left(100 \mathrm{Mhz} \mathrm{CDCl}_{3}\right) \delta 159.9,141.8,137.1,134.5,131.3,129.8$, 129.4, 128.5, 128.2, 125.9, 113.2, 58.7, 55.0, 18.1. IR (film, $\mathrm{cm}^{-1}$ ): v 2973.52, 2925.56, 2360.47, 1604.60. HRMS (ESI) Calculated for $\mathrm{C}_{21} \mathrm{H}_{28} \mathrm{LiO}_{4} \mathrm{Si}[\mathrm{M}+\mathrm{Li}]^{+}$379.1912, measured 379.1910.

Minor product: triethoxy[(E)-(1-(4-methoxyphenyl)-2-phenylvinyl)]silane relevant signals: ${ }^{1} \mathbf{H}$ NMR $\left(400 \mathrm{Mhz}, \mathrm{CDCl}_{3}\right) \delta 6.89(\mathrm{~d}, J=9 \mathrm{~Hz}, 2 \mathrm{H}), 6.89(\mathrm{~d}, J=8.7 \mathrm{~Hz}, 2 \mathrm{H})$. Other signals are overlapped with the $E$-isomer.

\section{(3-(2-(dimethylsilyl)phenyl)prop-1-yn-1-yl)trimethylsilane (4)}


To activated magnesium turnings $(0.63 \mathrm{~g}, 2.59 \mathrm{mmol})$ was added (3-(2bromophenyl)prop-1-yn-1-yl)trimethylsilane $(630 \mathrm{mg}, 2.36 \mathrm{mmol}$ ) in dry THF ( $4 \mathrm{~mL})$ under an atmosphere of argon. Chlorodimethylsilane $(446 \mathrm{mg}$, $4.71 \mathrm{mmol}$ ) was added to the stirred solution via a syringe. After $24 \mathrm{~h}$, the<smiles>CC#CCc1ccccc1[SiH](C)C</smiles>
reaction mixture was quenched by addition of $10 \mathrm{~mL}$ of water, extracted three times with diethyl ether. The combined organic layer was washed with aqueous $\mathrm{NaCl}$ and $\mathrm{NaHCO}_{3}$ and dried over $\mathrm{K}_{2} \mathrm{CO}_{3}$. Purification by silica gel flash-chromatography (pentane 100\%) afforded the title compound 4 as a yellow oil $\left(222 \mathrm{mg}, 30 \%\right.$ yield) ${ }^{1} \mathbf{H}$ NMR $\left(400 \mathbf{M H z} \mathbf{C D C l}_{3}\right) \delta 7.57(\mathrm{~m}, 1 \mathrm{H}), 7.53-7.50(\mathrm{~m}$, $1 \mathrm{H}), 7.41(\mathrm{~m}, 2 \mathrm{H}), 7.31-7.25(\mathrm{~m}, 1 \mathrm{H}), 4.60(\mathrm{~m}, 1 \mathrm{H}), 3.80(\mathrm{~s}, 2 \mathrm{H}), 0.41(\mathrm{~d}, \mathrm{~J}=3.8 \mathrm{~Hz}, 6 \mathrm{H}), 0.21(\mathrm{~s}$, 9H). ${ }^{13} \mathrm{C}$ NMR (100 Mhz, $\mathrm{CDCl}_{3}$ ) $\delta 141.9,135.6,134.6,129.7,127.9,126.0,104.5,87.3,26.6,-0.0$, 3.4. HRMS (ESI) Calculated for $\mathrm{C}_{14} \mathrm{H}_{22} \mathrm{NaSi}_{2}[\mathrm{M}+\mathrm{Na}]^{+} 269.1152$, measured 269.1163.

\section{1,1-dimethyl-2-((trimethylsilyl)methyl)-1H-benzo[b]silole (5)}

Under argon, a glass vial $(0.5-2 \mathrm{~mL})$ was charged with (3-(2(dimethylsilyl)phenyl)prop-1-yn-1-yl) $(4,100 \mathrm{mg}, 0.4 \mathrm{mmol})$ and $(7.5 \mathrm{mg}$, $0.02 \mathrm{mmol})$ of $\mathrm{HCo}\left(\mathrm{PMe}_{3}\right)_{4}$. The vial was sealed followed by the addition of<smiles>C[Si]1(C)C(C[As](C)(C)C)=Cc2ccccc21</smiles>
freshly distilled and degassed toluene $(1 \mathrm{ml})$. The reaction was heated in an oil bath at $110{ }^{\circ} \mathrm{C}$ for $12 \mathrm{~h}$. The reaction mixture was diluted with ethyl acetate $(2 \mathrm{~mL})$, filtered through a plug of celite and washed with ethyl acetate $(3 \times 10 \mathrm{ml})$. The crude product was purified by silica chromatography (pentane 100\%) to afford the title compound 5 as a yellow oil $(80 \mathrm{mg}, 80$ $\%$ yield). ${ }^{1} \mathrm{H}$ NMR $\left(400 \mathrm{MHz}, \mathrm{CDCl}_{3}\right) \delta 7.40-7.36(\mathrm{~m}, 1 \mathrm{H}), 7.24-7.18(\mathrm{~m}, 1 \mathrm{H}), 7.07-7.01(\mathrm{~m}, 2 \mathrm{H})$, $6.71(\mathrm{~s}, 1 \mathrm{H}), 1.80(\mathrm{~d}, \mathrm{~J}=1.3 \mathrm{~Hz}, 2 \mathrm{H}), 0.24(\mathrm{~d}, \mathrm{~J}=0.6 \mathrm{~Hz}, 6 \mathrm{H}), 0.00(\mathrm{~s}, 9 \mathrm{H}) .{ }^{13} \mathrm{C} \mathbf{N M R}\left(100 \mathrm{Mhz}, \mathrm{CDCl}_{3}\right)$ $\delta 150.9,147.4,141.9,138.4,132.4,130.7,126.1,123.3,23.5,-0.0,-2.8$. HRMS (ESI) Calculated for $\mathrm{C}_{14} \mathrm{H}_{22} \mathrm{NaSi}_{2}[\mathrm{M}+\mathrm{Na}]^{+}$269.1152, measured 269.1159.

\section{Preparation of dihydrido cobalt(III) complex 6.}

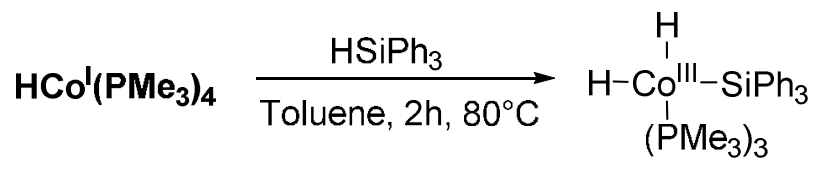

$6,60 \%$

Under argon atmosphere, an oven-dried Schlenk tube containing a magnetic stir bar was charged with cobalt(I) complex $\mathrm{HCo}\left(\mathrm{PMe}_{3}\right)_{4}(100 \mathrm{mg}, 0.274 \mathrm{mmol})$ and triphenylsilane $(71 \mathrm{mg}, 0.274$ $\mathrm{mmol})$. The Schlenk tube was then capped with a rubber septum and then freshly distilled and degassed toluene $(5 \mathrm{~mL})$ was added via syringe through the septum. The reaction mixture was stirred in an oil bath at $80^{\circ} \mathrm{C}$ for $2 \mathrm{~h}$, after which time the solvent was removed $i$. v. until dryness. Next, distilled and degassed pentane $(5 \mathrm{~mL})$ was added and the solution was filtered via cannula from the solid precipitate (this sequence was repeated three times). Finally, after complete removal of the solvent complex 6 is obtained as a yellow solid ( $90 \mathrm{mg}, 60 \%$ yield). ${ }^{1} \mathbf{H}$ NMR (400 
MHz, $\left.\mathrm{C}_{6} \mathrm{D}_{6}\right) \delta 7.94(\mathrm{~d}, J=7.3 \mathrm{~Hz}, 6 \mathrm{H}), 7.32-7.17(\mathrm{~m}, 9 \mathrm{H}), 0.95(\mathrm{~s}, 27 \mathrm{H}),-14.72(\mathrm{~s}, 2 \mathrm{H}) .{ }^{13} \mathrm{C} \mathrm{NMR}$ (151 MHz, $\left.\mathrm{C}_{6} \mathrm{D}_{6}\right) \delta 150.1,136.7,127.0,126.5,23.8 .{ }^{31} \mathrm{P}$ NMR (162 MHz, $\mathrm{C}_{6} \mathrm{D}_{6}$ ) $\delta 4.72 .{ }^{29}$ Si NMR (119 $\left.\mathrm{MHz}, \mathrm{C}_{6} \mathrm{D}_{6}\right) \delta 9.69\left({ }^{2} \mathrm{~J}_{\mathrm{Si}-\mathrm{P}}=24,79 \mathrm{~Hz}\right)$.

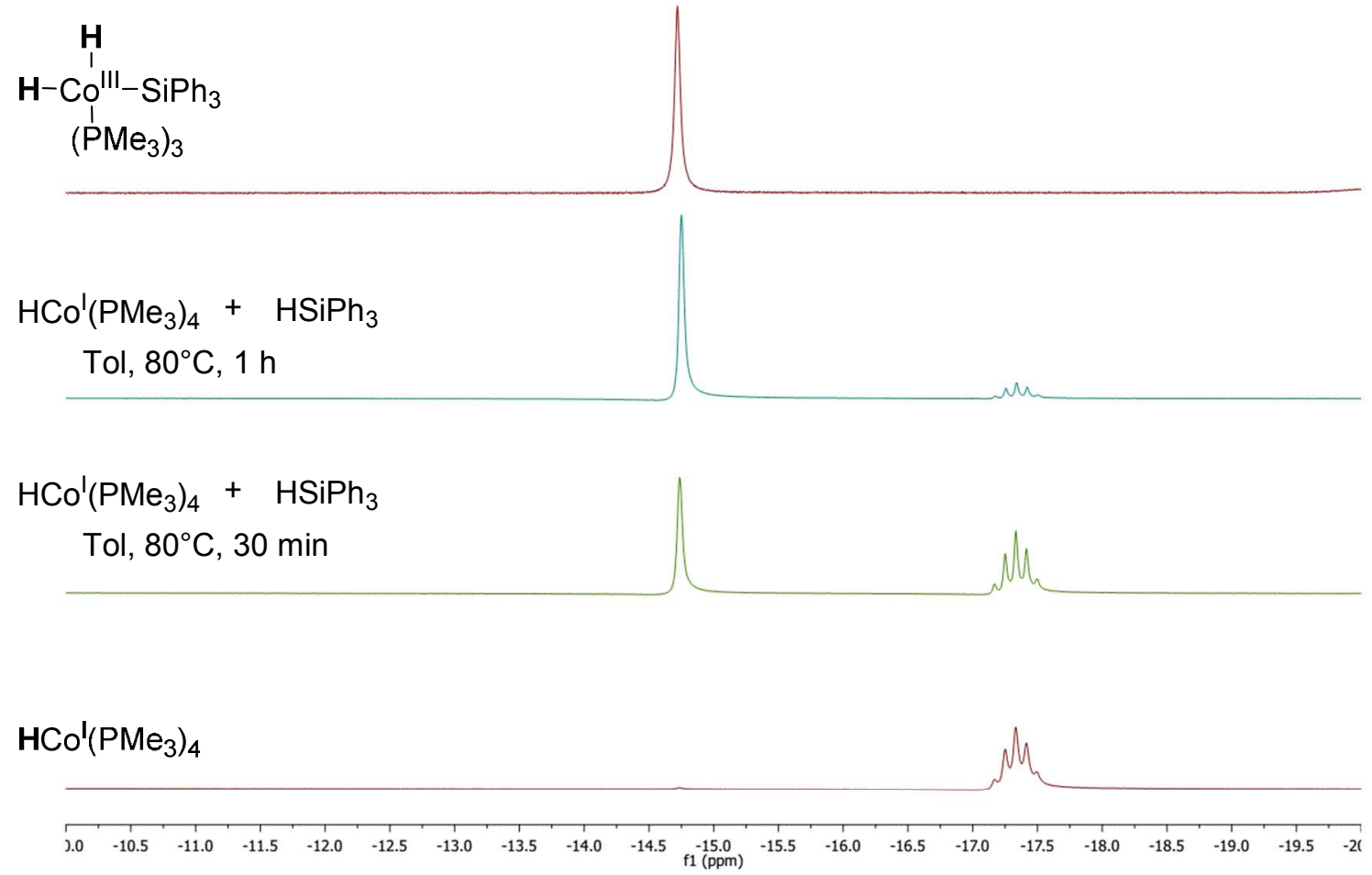

Figure S1. Formation of dihydridocobalt(III) 6 complex followed by ${ }^{1} \mathrm{H}$ NMR (metal-H). 


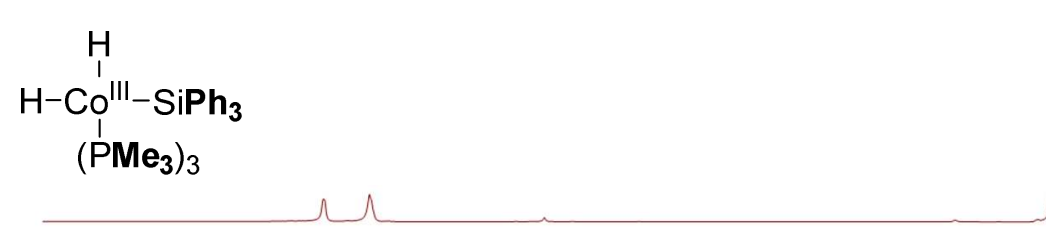

$$
\mathrm{HCo}^{\prime}\left(\mathrm{PMe}_{3}\right)_{4}+\mathrm{HSiPh}_{3}
$$

Tol, $80^{\circ} \mathrm{C}, 1 \mathrm{~h}$

$$
\mathrm{HCo}^{\prime}\left(\mathrm{PMe}_{3}\right)_{4}+\mathrm{HSiPh}_{3}
$$

$\mathrm{Tol}, 80^{\circ} \mathrm{C}, 30 \mathrm{~min}$

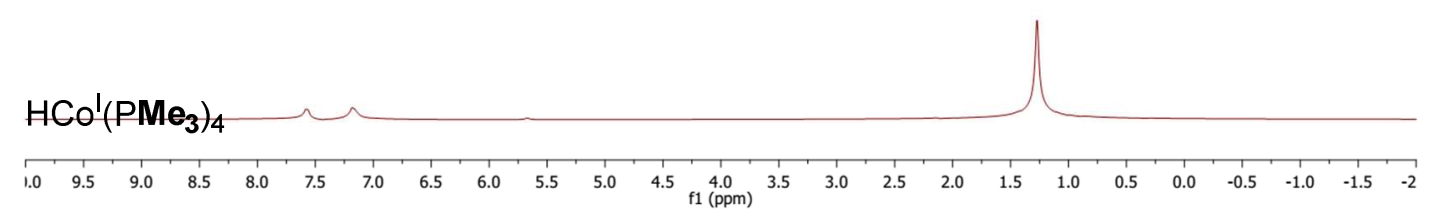

Figure S2. Formation of dihydridocobalt(III) 6 complex followed by ${ }^{1} \mathrm{H}$ NMR (methyl and phenyl H).

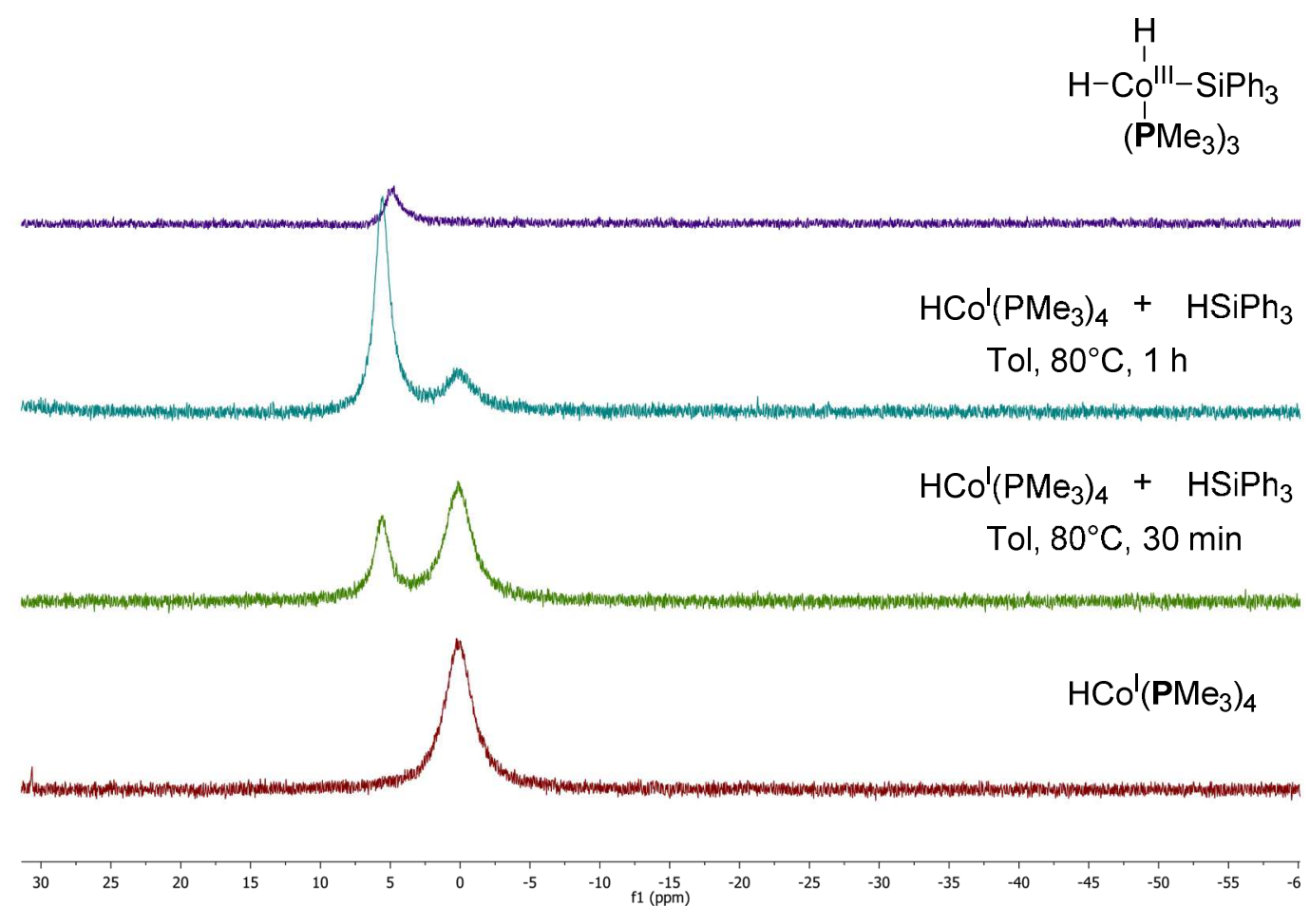

Figure S3. Formation of dihydridocobalt(III) complex 6 followed by ${ }^{31} \mathrm{P}$ NMR. 


\section{Theorical methods}

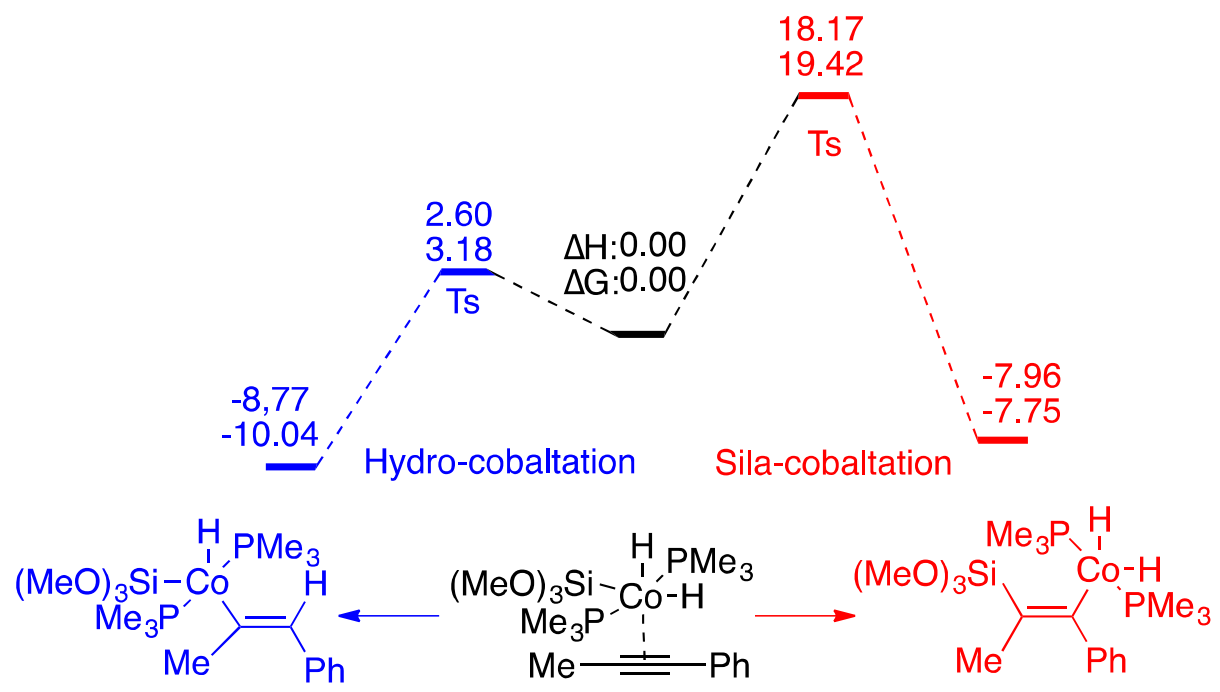

Figure S1. DFT calculated potential energy surface for the hydro-cobaltation and the sila-cobaltation.

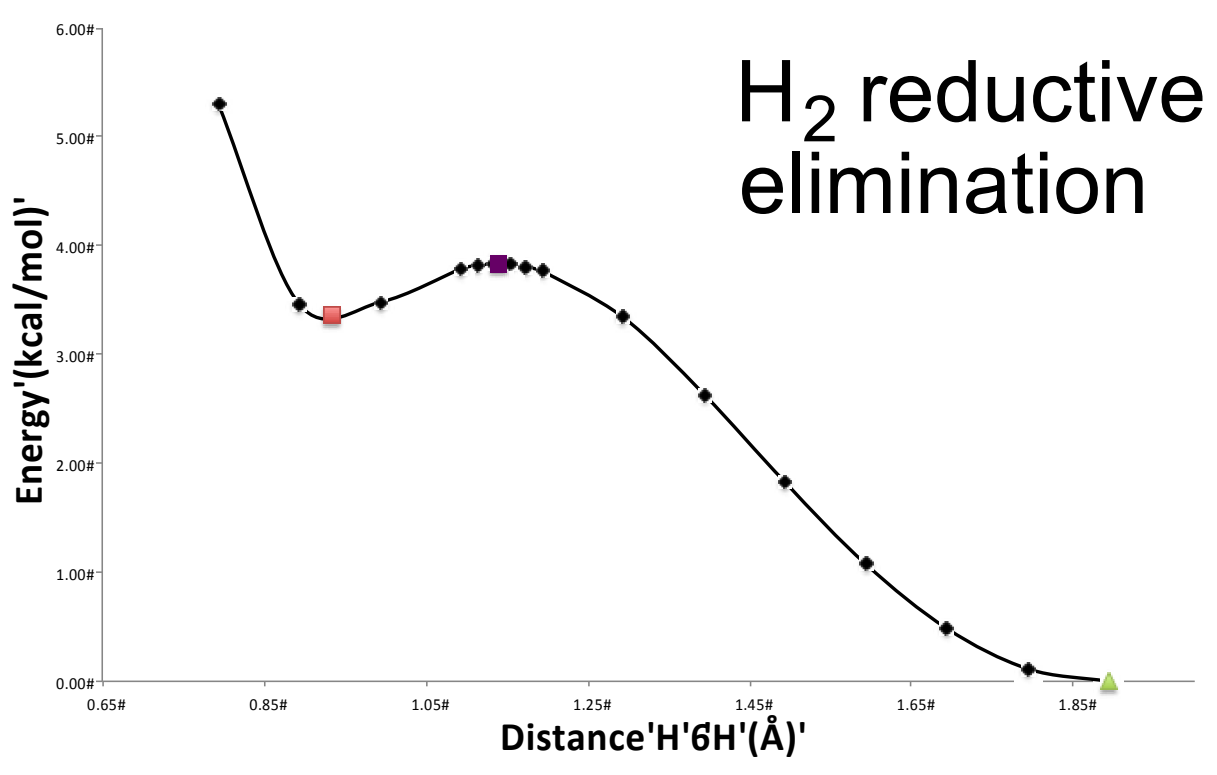

Figure S2. DFT calculated potential energy surface for $\mathrm{H}_{2}$ reductive elimination. 


\section{Reagent}

62

$\begin{array}{llll}\text { P } & 0.013349 & 2.270601 & -0.735109\end{array}$

C $0.031579 \quad 3.271097 \quad 0.813155$

C $1.494901 \quad 2.930518-1.617305$

C $-1.360130 \quad 3.053500-1.684210$

Co $\quad-0.067962 \quad 0.046576 \quad-0.505509$

$\begin{array}{llll}\text { P } & 0.076115 & -2.142362 & -0.905440\end{array}$

C $1.531823 \quad-2.692215 \quad-1.897655$

C $0.191293-3.2291550 .577056$

C $-1.310161 \quad-2.891529 \quad-1.864351$

Si $\quad-2.146064 \quad-0.108755 \quad 0.325119$

$\begin{array}{llll}0 & -3.463103 & -0.557139 & -0.668232\end{array}$

$\begin{array}{llll}0 & -2.641887 & 1.383269 & 0.990847\end{array}$

$\begin{array}{llll}\mathrm{O} & -2.347485 & -1.322553 & 1.497112\end{array}$

C $\quad-3.889039 \quad 1.606867 \quad 1.608908$

$\begin{array}{llll}\text { C } & -3.904464 & 0.092755 & -1.824347\end{array}$

C $-3.550462 \quad-1.923421 \quad 1.919804$

$\begin{array}{llll}H & 0.741966 & 0.106889 & -1.760647\end{array}$

H $\quad-1.1355820 .100043 \quad-1.515883$

$\begin{array}{llll}H & 0.900395 & 2.983531 & 1.422551\end{array}$

H $\quad-0.888169 \quad 3.041934 \quad 1.368343$

$\begin{array}{llll}H & 0.083337 & 4.348603 & 0.590337\end{array}$

H $1.526114 \quad 2.517857 \quad-2.636321$

H $2.406279 \quad 2.612201 \quad-1.091423$

H $\quad 1.470518 \quad 4.031111-1.667988$

H $\quad-1.418415 \quad 2.604672 \quad-2.686875$

H $\quad-1.210310 \quad 4.140575 \quad-1.781762$

H $\quad-2.301032 \quad 2.858077 \quad-1.151239$

H $\quad 1.529860-3.786130-2.030680$

H $\quad 2.459114 \quad-2.389294 \quad-1.391507$

H $\quad 1.503153 \quad-2.204077 \quad-2.882834$

H $1.098940-2.971860 \quad 1.142032$

H $0.223789-4.293578 \quad 0.296039$

H $\quad-0.683334-3.022381 \quad 1.209261$

H $\quad-1.164213-3.975651-1.993768$

H $-1.355027 \quad-2.413411-2.854695$

H $\quad-2.262850-2.696699-1.352245$

H $\quad-4.716088 \quad 1.098522 \quad 1.080365$

$\begin{array}{llll}H & -4.102493 & 2.689810 & 1.615853\end{array}$

$\begin{array}{llll}H & -3.892043 & 1.257841 & 2.658634\end{array}$

H $\quad-3.0847640 .269678-2.549502$

H $\quad-4.368764 \quad 1.074798 \quad-1.605388$ 


$\begin{array}{cccc}\text { H } & -4.664911 & -0.530791 & -2.325704 \\ \text { H } & -3.334953 & -2.942440 & 2.286832 \\ \text { H } & -4.286382 & -1.994925 & 1.101843 \\ \text { H } & -4.012997 & -1.361748 & 2.753512 \\ \text { C } & 1.577329 & 0.016108 & 0.665821 \\ \text { C } & 0.565289 & 0.044106 & 1.442754 \\ \text { C } & 0.050419 & 0.104579 & 2.829836 \\ \text { C } & 5.083019 & 0.039594 & -0.761955 \\ \text { C } & 3.688054 & 0.060303 & -0.687053 \\ \text { C } & 3.025093 & -0.011505 & 0.555311 \\ \text { C } & 3.820566 & -0.111373 & 1.721724 \\ \text { C } & 5.212085 & -0.133077 & 1.643632 \\ \text { C } & 5.853103 & -0.056796 & 0.400696 \\ \text { H } & 5.572681 & 0.098400 & -1.737987 \\ \text { H } & 3.082925 & 0.132909 & -1.592575 \\ \text { H } & 3.329488 & -0.174007 & 2.695515 \\ \text { H } & 5.803712 & -0.211221 & 2.559995 \\ \text { H } & 6.944311 & -0.074397 & 0.340948 \\ \text { H } & -0.553669 & -0.785601 & 3.060217 \\ \text { H } & 0.876447 & 0.183852 & 3.557414 \\ \text { H } & -0.621109 & 0.967581 & 2.965897\end{array}$

\section{TS sila-cobaltation}

\section{2}

$\begin{array}{cccc}\text { P } & -0.343096 & 2.671951 & 0.113807 \\ \text { C } & 1.133822 & 3.510288 & -0.613210 \\ \text { C } & -1.623535 & 3.014359 & -1.179201 \\ \text { C } & -0.823482 & 3.848924 & 1.450099 \\ \text { Co } & -0.015348 & 0.603811 & 0.774721 \\ \text { P } & 0.180750 & -1.072672 & 2.207143 \\ \text { C } & -1.438116 & -1.495743 & 2.990661 \\ \text { C } & 0.762487 & -2.750872 & 1.684143 \\ \text { C } & 1.233177 & -0.754457 & 3.687908 \\ \text { Si } & 1.846824 & -0.524117 & -0.809636 \\ \text { O } & 2.958945 & -0.663015 & 0.450501 \\ \text { O } & 2.469362 & 0.666111 & -1.825766 \\ \text { O } & 2.035011 & -2.045315 & -1.488508 \\ \text { C } & 3.650150 & 0.528038 & -2.582544 \\ \text { C } & 3.734207 & 0.348502 & 1.047210 \\ \text { C } & 3.146547 & -2.906534 & -1.452255 \\ \text { H } & -0.941494 & 1.034989 & 1.813529 \\ \text { H } & 1.139692 & 1.326100 & 1.455661\end{array}$




\begin{tabular}{|c|c|c|c|}
\hline - & 1.552951 & 2.875987 & -1.408047 \\
\hline$H$ & 1.899233 & 3.611181 & 0.170286 \\
\hline & 0.881086 & 4.506547 & -1.010551 \\
\hline & -2.605092 & 2.655639 & -0.837836 \\
\hline & -1.364606 & 2.462525 & -2.095279 \\
\hline & -1.687723 & 4.089281 & -1.412803 \\
\hline & -1.810776 & 3.570956 & 1.847025 \\
\hline & -0.858029 & 4.886666 & 1.081567 \\
\hline & -0.092499 & 3.773667 & 2.268066 \\
\hline & -1.330932 & -2.317672 & 3.716544 \\
\hline & -2.153080 & -1.789759 & 2.208537 \\
\hline & -1.840224 & -0.608737 & 3.501313 \\
\hline & 0.227165 & -3.058627 & 0.774090 \\
\hline & 0.591110 & -3.493773 & 2.479166 \\
\hline & 1.834782 & -2.696135 & 1.453819 \\
\hline & 1.180089 & -1.582434 & 4.413287 \\
\hline & 0.899331 & 0.176609 & 4.168715 \\
\hline & 2.274375 & -0.61 & 3.364099 \\
\hline & 4.483433 & 0.101184 & -1.993815 \\
\hline & 3.964516 & 1.522445 & -2.940151 \\
\hline & 3.493044 & -0.11 & 54793 \\
\hline & 3.098226 & 1.074561 & 1.583829 \\
\hline & 4.334662 & 0.903762 & 0.304398 \\
\hline & 4.425527 & -0.122052 & 1.766002 \\
\hline & 2.809872 & -3.934297 & -1.229848 \\
\hline & 3.879705 & -2.609697 & -0.683849 \\
\hline & 3.655566 & -2.926515 & -2.433908 \\
\hline$C$ & -1.160725 & -0.379035 & -0.406926 \\
\hline$C$ & -0.096825 & -0.442057 & -1.219273 \\
\hline 0 & -0.151412 & -0.440024 & -2.747664 \\
\hline C & -4.925459 & -0.255101 & -0.146167 \\
\hline$C$ & -3.572857 & 0.012014 & 0.064921 \\
\hline . & -2.566732 & -0.678365 & -0.648513 \\
\hline C & -2.981871 & -1.693173 & -1.543745 \\
\hline C & -4.334978 & -1.973824 & -1.739252 \\
\hline C & -5.314736 & -1.249544 & -1.051181 \\
\hline $\mathrm{H}$ & -5.683046 & 0.302758 & 0.411351 \\
\hline $\mathrm{H}$ & -3.261042 & 0.755296 & 0.801891 \\
\hline $\mathrm{H}$ & -2.226959 & -2.277593 & -2.073567 \\
\hline $\mathrm{H}$ & -4.628402 & -2.766544 & -2.433109 \\
\hline $\mathrm{H}$ & -6.374064 & -1.470106 & -1.206446 \\
\hline $\mathrm{H}$ & 0.278029 & -1.362709 & -3.169194 \\
\hline $\mathrm{H}$ & -1.196536 & -0.352448 & -3.081198 \\
\hline & 0.421640 & 0.401268 & -3.165471 \\
\hline
\end{tabular}




\section{Product sila-cobaltation}

$\begin{array}{cccc}\text { 62 } & & & \\ \text { P } & 0.479071 & 2.520894 & -0.607064 \\ \text { C } & 2.222606 & 3.085006 & -0.355991 \\ \text { C } & 0.468756 & 2.303224 & -2.446865 \\ \text { C } & -0.464705 & 4.093991 & -0.414779 \\ \text { Co } & -0.196407 & 0.910427 & 0.720353 \\ \text { P } & -1.155589 & -0.158101 & 2.390519 \\ \text { C } & -2.993885 & -0.040568 & 2.460226 \\ \text { C } & -0.875429 & -1.982644 & 2.511293 \\ \text { C } & -0.684869 & 0.385506 & 4.094501 \\ \text { Si } & 1.953241 & -1.081463 & -0.378699 \\ \text { O } & 1.689717 & -0.059601 & 0.948489 \\ \text { O } & 3.155601 & -0.252339 & -1.214289 \\ \text { O } & 2.607809 & -2.531291 & 0.109261 \\ \text { C } & 3.680724 & -0.594031 & -2.477368 \\ \text { C } & 2.698822 & 0.332171 & 1.868312 \\ \text { C } & 3.930582 & -2.854663 & 0.466404 \\ \text { H } & -1.481130 & 1.560003 & 0.580413 \\ \text { H } & 0.136787 & 1.988406 & 1.752795 \\ \text { H } & 2.899313 & 2.253669 & -0.603186 \\ \text { H } & 2.368106 & 3.362896 & 0.697740 \\ \text { H } & 2.461237 & 3.949020 & -0.997122 \\ \text { H } & -0.547745 & 2.070956 & -2.794399 \\ \text { H } & 1.112699 & 1.449765 & -2.703122 \\ \text { H } & 0.833623 & 3.204612 & -2.965401 \\ \text { H } & -1.518323 & 3.922652 & -0.679807 \\ \text { H } & -0.054260 & 4.893242 & -1.052399 \\ \text { H } & -0.427212 & 4.403337 & 0.639385 \\ \text { H } & -3.405498 & -0.608414 & 3.310160 \\ \text { H } & -3.416486 & -0.424875 & 1.520706 \\ \text { H } & -3.279127 & 1.017474 & 2.555095 \\ \text { H } & -1.295351 & -2.469820 & 1.620441 \\ \text { H } & -1.340874 & -2.408901 & 3.414397 \\ \text { H } & 0.205151 & -2.189351 & 2.526101 \\ \text { H } & -1.215217 & -0.189261 & 4.871352 \\ \text { H } & -0.914686 & 1.455238 & 4.203804 \\ \text { H } & 0.399654 & 0.258021 & 4.231147 \\ \text { H } & 4.567388 & 0.029860 & -2.675684 \\ \text { H } & 2.950172 & -0.413043 & -3.287146 \\ & 3.991975 & -1.653906 & -2.530936\end{array}$




\begin{tabular}{|c|c|c|c|}
\hline$H$ & 2.286919 & 1.123809 & 2.507211 \\
\hline H & 3.587642 & 0.719975 & 1.343535 \\
\hline & 3.002596 & -0.521204 & 499717 \\
\hline & 4.203332 & -3.829147 & 0.026672 \\
\hline & 4.030994 & -2.942061 & 1.563537 \\
\hline & 4.658914 & -2.101797 & 0.114822 \\
\hline & -0.660731 & -0.489996 & -0.663478 \\
\hline & 0.315616 & -1.316462 & -1.157971 \\
\hline & 0.170293 & -2.385580 & -2.233774 \\
\hline & -4.173712 & 0.219402 & -1.999405 \\
\hline & -2.845228 & 0.394796 & -1.609349 \\
\hline & -2.070787 & -0.673873 & -1.109059 \\
\hline & -2.717642 & -1.924550 & -0.990818 \\
\hline & -4.056858 & -2.098374 & -1.353714 \\
\hline$C$ & -4.793422 & -1.028598 & -1.869060 \\
\hline 1 & -4.736136 & 1.069519 & -2.396265 \\
\hline & -2.392609 & 1.384145 & -1.683609 \\
\hline F & -2.156644 & -2.777228 & -0.600288 \\
\hline$\Pi$ & -4.524362 & -3.080717 & -1.238850 \\
\hline & -5.838330 & -1.162901 & -2.160644 \\
\hline & 0.207531 & -3.406088 & -1.811290 \\
\hline & -0.773164 & -2.289505 & -2.790689 \\
\hline & 0.992699 & -2.334018 & -2.970075 \\
\hline
\end{tabular}

\section{TS Hydro-cobaltation}

62

$\begin{array}{cccc}\text { P } & -0.092030 & 2.243799 & -0.758647 \\ \text { C } & 0.100581 & 3.281113 & 0.753066 \\ \text { C } & 1.283588 & 2.864631 & -1.826887 \\ \text { C } & -1.550305 & 3.022281 & -1.572607 \\ \text { Co } & -0.051879 & 0.033832 & -0.484560 \\ \text { P } & -0.072882 & -2.149813 & -0.898484 \\ \text { C } & 1.311700 & -2.700837 & -1.992003 \\ \text { C } & 0.113897 & -3.273550 & 0.550539 \\ \text { C } & -1.522645 & -2.861374 & -1.786061 \\ \text { Si } & -2.138639 & -0.069177 & 0.360598 \\ \text { O } & -3.465731 & -0.458899 & -0.642433 \\ \text { O } & -2.606145 & 1.398547 & 1.109582 \\ \text { O } & -2.351597 & -1.303845 & 1.515815 \\ \text { C } & -3.826512 & 1.607555 & 1.780278 \\ \text { C } & -3.844342 & 0.147824 & -1.844219 \\ C & -3.561259 & -1.873274 & 1.957760\end{array}$




\begin{tabular}{|c|c|c|c|}
\hline $\mathrm{H}$ & 1.306249 & 0.066747 & -1.112436 \\
\hline$H$ & -0.584963 & 0.078965 & -1.875347 \\
\hline & 1.023231 & 2.995305 & 1.278701 \\
\hline & -0.760801 & 3.073776 & 1.402546 \\
\hline r & 0.138365 & 4.353881 & 0.505967 \\
\hline & 1.215969 & 2.387170 & -2.815637 \\
\hline & 2.250315 & 2.591153 & -1.379378 \\
\hline & 1.237112 & 3.959221 & -1.946131 \\
\hline & -1.710145 & 2.563606 & -2.559072 \\
\hline & -1.400054 & 4.106741 & -1.695094 \\
\hline & -2.432817 & 2.840391 & -0.945250 \\
\hline & 1.265694 & -3.78 & -2.17 \\
\hline & 2.274112 & -2.456799 & -1.519316 \\
\hline & 1.251620 & -2.162756 & -2.949581 \\
\hline & 1.051441 & -3.035591 & 4453 \\
\hline & 0.124407 & -4.331789 & 0.245397 \\
\hline & -0.726681 & -3.077205 & 1.230584 \\
\hline & -1.405655 & -3.948154 & -1.921791 \\
\hline & -1.602677 & -2.379603 & -2.771972 \\
\hline & -2.444566 & -2.644894 & -1.230040 \\
\hline & -4.675636 & 1.11 & 1.26 \\
\hline (1) & -4.037601 & 2.690416 & 1.826977 \\
\hline & -3.791843 & 1.229037 & 2.819450 \\
\hline & -3.003360 & 843 & 0575 \\
\hline & -4.249581 & 1.167809 & -1.693002 \\
\hline & -4.637499 & -0.454528 & -2.321040 \\
\hline H & -3.381599 & -2.920539 & 2.260490 \\
\hline $\mathrm{H}$ & -4.333201 & -1.868867 & 1.169532 \\
\hline & -3.964466 & -1.339521 & 2.839396 \\
\hline 0 & 1.699480 & 0.008110 & 0.598203 \\
\hline 0 & 0.700395 & 0.014173 & 1.397062 \\
\hline 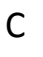 & 0.241346 & 0.027223 & 2.803084 \\
\hline & 5.235557 & 0.007135 & -0.783751 \\
\hline$C$ & 3.839389 & 0.023420 & -0.737599 \\
\hline & 3.151008 & -0.010452 & 0.490916 \\
\hline C & 3.922238 & -0.063168 & 1.676276 \\
\hline$C$ & 5.314471 & -0.079427 & 1.627108 \\
\hline$C$ & 5.982001 & -0.044223 & 0.396182 \\
\hline $\mathrm{H}$ & 5.743252 & 0.034782 & -1.751710 \\
\hline $\mathrm{H}$ & 3.261482 & 0.063349 & -1.663896 \\
\hline $\mathrm{H}$ & 3.412789 & -0.092420 & 2.641685 \\
\hline $\mathrm{H}$ & 5.886154 & -0.120812 & 2.558246 \\
\hline $\mathrm{H}$ & 7.074123 & -0.057542 & 0.359614 \\
\hline & -0.430106 & -0.823772 & 2.993416 \\
\hline
\end{tabular}



H $\quad 1.079826 \quad 0.002648 \quad 3.520026$
$\begin{array}{llll}H & -0.363289 & 0.927762 & 2.999894\end{array}$

\section{Product Hydro-cobaltation}

62

$\begin{array}{llll}\text { P } & -0.359269 & 2.248616 & -0.833783\end{array}$

$\begin{array}{llll}\text { C } & -0.046719 & 3.318078 & 0.632620\end{array}$

C $1.027162 \quad 2.713641-1.965046$

C $-1.777345 \quad 3.095529-1.658083$

$\begin{array}{llll}\text { Co } & -0.508743 & 0.036121 & -0.578397\end{array}$

P $\quad-0.376326 \quad-2.168580 \quad-0.768041$

$\begin{array}{llll}\text { C } & 0.823510 & -2.611089 & -2.102507\end{array}$

C $0.214522 \quad-3.161563 \quad 0.665214$

C $-1.863311 \quad-3.120744 \quad-1.313743$

Si $\quad-2.414036 \quad-0.044765 \quad 0.429824$

$\begin{array}{llll}\text { O } & -3.812279 & -0.837302 & -0.113486\end{array}$

$\begin{array}{llll}0 & -2.927989 & 1.525194 & 0.824435\end{array}$

$\begin{array}{llll}0 & -2.175748 & -0.891767 & 1.873456\end{array}$

$\begin{array}{llll}\text { C } & -3.833401 & 1.799082 & 1.870464\end{array}$

C $-4.580542 \quad-0.447248 \quad-1.219554$

C $\quad-3.121561 \quad-1.562560 \quad 2.676039$

H $\quad 2.384446 \quad-0.000007 \quad-1.450666$

H $\quad-1.834677 \quad 0.109840 \quad-1.351585$

$\begin{array}{llll}H & 0.904042 & 3.026647 & 1.100579\end{array}$

H $\quad-0.863330 \quad 3.162333 \quad 1.350983$

$\begin{array}{llll}H & -0.000668 & 4.380884 & 0.347187\end{array}$

H $0.915235 \quad 2.189527 \quad-2.926872$

H $1.985294 \quad 2.417429-1.518026$

H $\quad 1.025962 \quad 3.799258-2.151893$

$\begin{array}{llll}\mathrm{H} & -1.958311 & 2.623400 & -2.635492\end{array}$

H $-1.572196 \quad 4.167711-1.807179$

H $\quad-2.668860 \quad 2.964265 \quad-1.031155$

H $\quad 0.868921 \quad-3.703485-2.237445$

H $1.820085-2.233159-1.838229$

H $\quad 0.509721 \quad-2.148865-3.051105$

H $\quad 1.222325 \quad-2.8284550 .948850$

H $\quad 0.235404 \quad-4.235962 \quad 0.424554$

H $\quad-0.463764 \quad-2.980674 \quad 1.511151$

H $\quad-1.619227 \quad-4.186531 \quad-1.447379$

H $\quad-2.218889-2.708902-2.270217$

H $\quad-2.674055-3.008541-0.581266$

H $\quad-4.744213 \quad 1.175069 \quad 1.806494$ 


$\begin{array}{llll}\text { H } & -4.141434 & 2.856458 & 1.809711 \\ \text { H } & -3.374759 & 1.633210 & 2.862527 \\ \text { H } & -3.969454 & -0.342444 & -2.137976 \\ \text { H } & -5.090683 & 0.520305 & -1.050638 \\ \text { H } & -5.353113 & -1.210902 & -1.411332 \\ \text { H } & -2.673716 & -2.490578 & 3.073534 \\ \text { H } & -4.029409 & -1.828494 & 2.109649 \\ \text { H } & -3.418994 & -0.940515 & 3.540848 \\ \text { C } & 2.438548 & 0.024035 & -0.351971 \\ \text { C } & 1.254458 & 0.019618 & 0.321588 \\ \text { C } & 1.175028 & -0.019948 & 1.836544 \\ \text { C } & 6.187393 & -0.474481 & -0.437990 \\ \text { C } & 4.838350 & -0.437571 & -0.787275 \\ \text { C } & 3.842917 & 0.014817 & 0.110912 \\ \text { C } & 4.286264 & 0.462759 & 1.376481 \\ \text { C } & 5.638607 & 0.428329 & 1.726736 \\ \text { C } & 6.598892 & -0.045677 & 0.828769 \\ \text { H } & 6.924482 & -0.837607 & -1.159957 \\ \text { H } & 4.533698 & -0.770485 & -1.784344 \\ \text { H } & 3.571724 & 0.869112 & 2.090321 \\ \text { H } & 5.945065 & 0.786361 & 2.713832 \\ \text { H } & 7.655473 & -0.070858 & 1.107484 \\ \text { H } & 0.215377 & -0.431352 & 2.181061 \\ \text { H } & 1.989331 & -0.609314 & 2.294387 \\ \text { H } & 1.241164 & 0.997179 & 2.265706\end{array}$

\section{TS $\mathrm{H} 2$ reductive elimination}

62
$\begin{array}{llll}\text { P } & -0.032363 & 2.286440 & -0.565314\end{array}$
$\begin{array}{llll}\text { C } & -0.107437 & 3.221643 & 1.017762\end{array}$
C $1.496328 \quad 2.981014 \quad-1.339606$
C $\quad-1.344544 \quad 3.100125-1.573948$
$\begin{array}{llll}\text { Co } & -0.033074 & 0.037887 & -0.461239\end{array}$
P $\quad 0.014845 \quad-2.199254 \quad-0.703799$
$\begin{array}{llll}\text { C } & 1.508419 & -2.821346 & -1.596848\end{array}$
$\begin{array}{llll}\text { C } & 0.042097 & -3.209494 & 0.832873\end{array}$
C $-1.331155-2.978151 \quad-1.696742$
Si $\quad-2.197506 \quad-0.074660 \quad 0.221674$
$\begin{array}{llll}0 & -3.438048 & -0.555511 & -0.860467\end{array}$
$\begin{array}{llll}0 & -2.781742 & 1.414678 & 0.837869\end{array}$
$\begin{array}{llll}O & -2.474364 & -1.252019 & 1.423049\end{array}$
C $\quad-4.067475 \quad 1.621616 \quad 1.373534$ 


\begin{tabular}{|c|c|c|c|}
\hline & -3.759798 & 0.063822 & -2.071099 \\
\hline & -3.707550 & -1.801337 & 1.824526 \\
\hline & 0.601662 & 0.089326 & -1.827480 \\
\hline & -0.537803 & 0.081197 & -1.867565 \\
\hline & 0.731581 & 2.919045 & 1.660377 \\
\hline & -1.056045 & 2.967018 & 1.508867 \\
\hline & -0.052005 & 4.306273 & 0.835057 \\
\hline & 1.591400 & 2.600024 & -2.367495 \\
\hline & 2.379906 & 2.654999 & -0.772794 \\
\hline & 1.465351 & 4.082480 & -1.364034 \\
\hline & -1.340982 & 2.679547 & -2.590826 \\
\hline & -1.185105 & 4.188561 & -1.633594 \\
\hline & -2.314602 & 2.890875 & -1.103601 \\
\hline & 1.481950 & -3.918719 & -1.694522 \\
\hline & 2.416951 & -2.526286 & -1.053523 \\
\hline & 1.548218 & -2.370685 & -2.599771 \\
\hline & 0.922485 & -2.932173 & 1.430230 \\
\hline & 0.082894 & -4.284806 & 0.598759 \\
\hline & -0.864749 & -2.972381 & 1.405571 \\
\hline & -1.195142 & -4.069347 & -1.761777 \\
\hline & -1.313317 & -2.553774 & -2.712461 \\
\hline & -2.307462 & -2.740671 & -1.252526 \\
\hline & -4.843743 & 1.049902 & 0.832548 \\
\hline & -4.325627 & 2.693319 & 1.304779 \\
\hline & -4.116585 & 1.334417 & 2.440917 \\
\hline & -2.874111 & 0.211908 & -2.722274 \\
\hline & -4.230823 & 1.056757 & -1.926888 \\
\hline & -4.478051 & -0.565553 & -2.625415 \\
\hline & -3.542080 & -2.823663 & 2.209615 \\
\hline & -4.426232 & -1.858957 & 0.989314 \\
\hline & -4.170544 & -1.213016 & 2.639425 \\
\hline & 1.693270 & 0.018129 & 0.595180 \\
\hline & 0.710771 & 0.012032 & 1.414570 \\
\hline & 0.197858 & 0.003401 & 2.801988 \\
\hline & 5.143782 & 0.032519 & -0.963087 \\
\hline & 3.752893 & 0.046682 & -0.833826 \\
\hline & 3.137145 & 0.005670 & 0.434255 \\
\hline & 3.979144 & -0.050934 & 1.570891 \\
\hline & 5.366700 & -0.066069 & 1.439659 \\
\hline & 5.958996 & -0.024201 & 0.171061 \\
\hline & 5.594613 & 0.065509 & -1.958849 \\
\hline & 3.115337 & 0.089696 & -1.719515 \\
\hline & 3.526443 & -0.083851 & 2.564662 \\
\hline & 5.993981 & -0.111037 & 2.334342 \\
\hline
\end{tabular}




$\begin{array}{rrrr}H & 7.047198 & -0.036260 & 0.069611 \\ H & -0.530318 & -0.809248 & 2.945673 \\ H & 1.012812 & -0.099146 & 3.539832 \\ H & -0.350029 & 0.935323 & 3.021580\end{array}$

Product $\mathrm{H} 2$ reductive elimination
62
P $\quad-0.042205 \quad 2.295056 \quad-0.507919$
C $-\begin{array}{llll}0.138530 & 3.208390 & 1.085796\end{array}$
C $1.501238 \quad 2.995714-1.249791$
C $-1.330998 \quad 3.129879-1.531410$
$\begin{array}{llll}\text { Co } & -0.033749 & 0.037488 & -0.440754\end{array}$
P $\quad-0.005685-2.213198 \quad-0.647988$
C $\quad 1.514920 \quad-2.852334 \quad-1.483518$
C $\quad-0.034057 \quad-3.2105880 .896574$
C $-1.322329-2.993992-1.679735$
Si $-2.221938-0.064833 \quad 0.183583$
$\begin{array}{llll}\text { O } & -3.450745 & -0.558085 & -0.910941\end{array}$
$\begin{array}{llll}0 & -2.824838 & 1.423268 & 0.788112\end{array}$
$\begin{array}{llll}\mathrm{O} & -2.508229 & -1.233551 & 1.392307\end{array}$
C $-4.111032 \quad 1.620320 \quad 1.325435$
C $-3.766931 \quad 0.072162-2.117213$
C $\quad-3.743605 \quad-1.780879 \quad 1.788996$
H $0.5124860 .092240 \quad-1.907868$
H $\quad-0.4203790 .085661 \quad-1.953030$
H $0.692355 \quad 2.896291 \quad 1.734303$
H $\quad-1.093991 \quad 2.950043 \quad 1.561193$
$\begin{array}{llll}H & -0.079001 & 4.295195 & 0.918223\end{array}$
H $1.6120892 .629246 \quad-2.281610$
H $\quad 2.375797 \quad 2.660463 \quad-0.674790$
H $\quad 1.472933 \quad 4.097483 \quad-1.261772$
$\begin{array}{llll}H & -1.310228 & 2.722380 & -2.553677\end{array}$
H $\quad-1.162782 \quad 4.217777 \quad-1.575778$
H $-2.311233 \quad 2.921113 \quad-1.083006$
H $1.486189-3.950503 \quad-1.571701$
H $\quad 2.404677 \quad-2.555776 \quad-0.910642$
H $1.594454-2.413330-2.489463$
H $0.830496-2.93623901 .517985$
H $0.006616 \quad-4.2874310 .669929$
H $\quad-0.956095 \quad-2.961763 \quad 1.439102$
H $-1.195234-4.087165-1.727590$
H $\quad-1.264298 \quad-2.583183 \quad-2.699745$ 


$\begin{array}{lrrr}\text { H } & -2.311597 & -2.741068 & -1.274266 \\ \text { H } & -4.882966 & 1.037419 & 0.790034 \\ \text { H } & -4.380828 & 2.688837 & 1.250509 \\ \text { H } & -4.154971 & 1.339525 & 2.394783 \\ \text { H } & -2.877938 & 0.231935 & -2.762190 \\ \text { H } & -4.243144 & 1.061695 & -1.966608 \\ \text { H } & -4.477972 & -0.554333 & -2.684039 \\ \text { H } & -3.582428 & -2.805592 & 2.170226 \\ \text { H } & -4.460836 & -1.833551 & 0.952108 \\ \text { H } & -4.206576 & -1.195007 & 2.605601 \\ \text { C } & 1.733381 & 0.013979 & 0.546267 \\ \text { C } & 0.761061 & -0.003040 & 1.384105 \\ \text { C } & 0.273246 & -0.035374 & 2.780955 \\ \text { C } & 5.179840 & 0.029009 & -1.020492 \\ \text { C } & 3.789662 & 0.040685 & -0.884814 \\ \text { C } & 3.177401 & 0.005481 & 0.385757 \\ \text { C } & 4.025730 & -0.041448 & 1.518653 \\ \text { C } & 5.412714 & -0.054021 & 1.381680 \\ \text { C } & 6.000085 & -0.018758 & 0.110470 \\ \text { H } & 5.626275 & 0.057127 & -2.018423 \\ \text { H } & 3.150247 & 0.076855 & -1.769836 \\ \text { H } & 3.577678 & -0.068474 & 2.514734 \\ \text { H } & 6.043866 & -0.091584 & 2.274049 \\ \text { H } & 7.087891 & -0.028582 & 0.004626 \\ \text { H } & -0.481381 & -0.825569 & 2.914741 \\ \text { H } & 1.094436 & -0.186890 & 3.503569 \\ \text { H } & -0.238181 & 0.908199 & 3.037013\end{array}$

In order to decipher how the cobalt catalyst $\left(\mathrm{HCo}\left(\mathrm{PMe}_{3}\right)_{4}\right)$ is working, and the interplay between substrates and ligands, some DFT analysis were performed. First of all, the replacement of one phosphine ligand by a silane (namely $\mathrm{HSi}(\mathrm{OMe})_{3}$ ). The results are summarized in the following table (S2). As can be seen, this process is highly exothermic. The best pathway is the one corresponding to $\mathrm{A} 2$ in which the silane is added trans to a phosphine and the two hydrogens are in cis configuration. Adding the silane in trans of one hydrogen is disfavorable as well as having the two hydrogens in trans. 


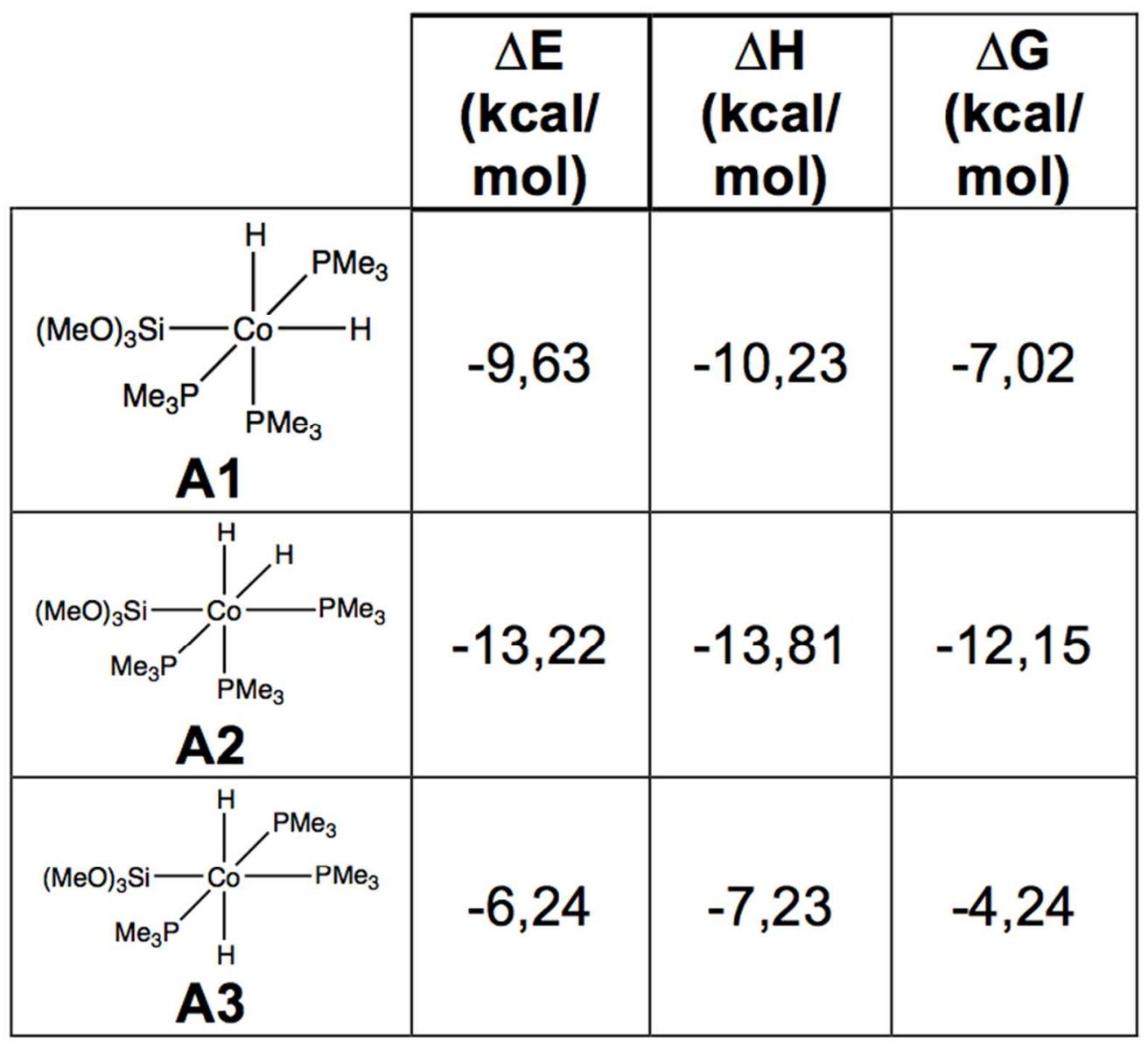

Table S2: energetics for the replacement of one phosphine by a silane.

We then tested the addition first of an alkyne on the same cobalt catalyst. The results are summarized in Table S3. As can be seen in this table, all the process (except one) are endothermic and the one exothermic is only slightly (by $1.61 \mathrm{kcal} / \mathrm{mol}$ ). Moreover, this stabilization is only due to entropic effects, something that is known to be not very reliable with static DFT calculations. Thus we can conclude that adding first a silane is a more favorable process than adding first an alkyne. The next step in the mechanism after silane addition and $\mathrm{Si}-\mathrm{H}$ bond breaking is thus to incorporate an alkyne by replacing a subsequent phosphine. This part of the mechanism is described in the main manuscript. 


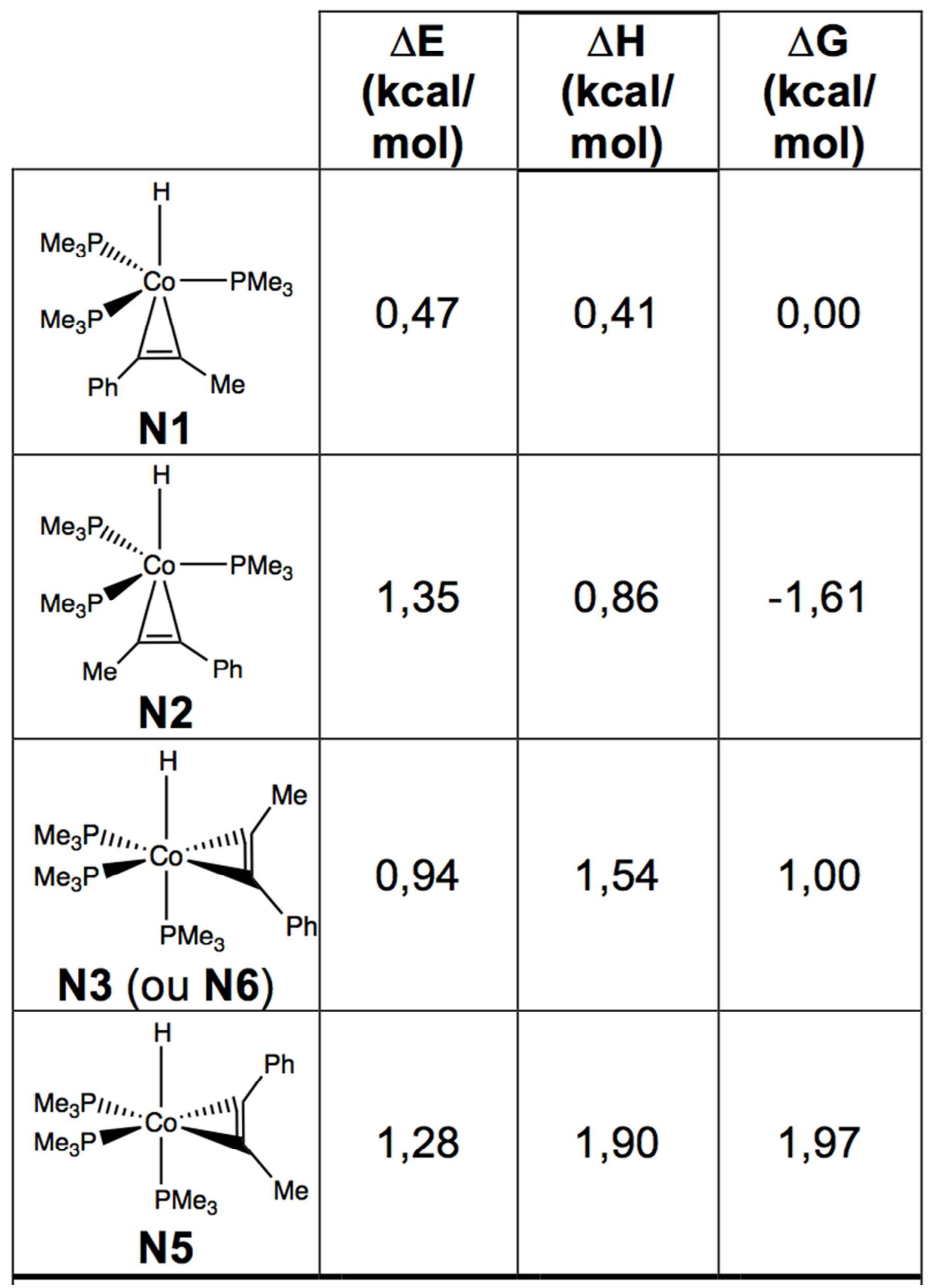

Table S3: energetics for the replacement of one phosphine by an alkyne 


\section{X-Ray Data}

Suitable X-ray quality crystals of compounds 3ha, 3ia and 3la (figures S4, S5 and S6 respectively) were grown, mounted and transferred into a cold nitrogen gas stream. Intensity data was collected with a Bruker Kappa-APEX2 system using fine-focus sealed tube Mo-Ka radiation. Unitcell parameters determination, data collection strategy and integration were carried out with the Bruker APEX2 suite of programs. Multi-scan absorption correction was applied. ${ }^{13}$ The structures were solved with SHELXT-2014 ${ }^{14}$ and refined anisotropically by full-matrix least-squares methods with SHELXL-2014 ${ }^{13}$ using the WinGX suite. ${ }^{15}$ The structures were deposited at the Cambridge Crystallographic Data Centre with numbers CCDC 1482313-1482315 and can be obtained free of charge via www.ccdc.cam.ac.uk.

Table S2. Crystal data and structure refinement.

\begin{tabular}{|c|c|c|c|c|c|c|}
\hline Compound & & 3ha & & 3ia & & 3la \\
\hline Empirical formula & & $\mathrm{C}_{31} \mathrm{H}_{34} \mathrm{O}_{2} \mathrm{Si}_{2}$ & & $\mathrm{C}_{34} \mathrm{H}_{34} \mathrm{O} \mathrm{Si}_{2}$ & & $\mathrm{C}_{34} \mathrm{H}_{30} \mathrm{Si}$ \\
\hline Formula weight & & 494.76 & & 514.79 & & 466.67 \\
\hline Temperature & & $200(1) \mathrm{K}$ & & $200(1) \mathrm{K}$ & & $200(1) \mathrm{K}$ \\
\hline Wavelength & & $0.71073 \AA$ & & $0.71073 \AA$ & & $0.71073 \AA$ \\
\hline Crystal system & & Monoclinic & & Triclinic & & Monoclinic \\
\hline Space group & & $\mathrm{P} 2{ }_{1} / \mathrm{c}$ & & $P-1$ & & $\mathrm{P} 2_{1} / \mathrm{c}$ \\
\hline \multirow{3}{*}{ Unit cell dimensions } & $a=16.1117(2)$ & $\alpha=90^{\circ}$ & $a=14.7880(3)$ & $\alpha=90^{\circ}$ & $\mathrm{a}=9.1784(2)$ & $\alpha=95.596(2)^{\circ}$ \\
\hline & $\mathrm{b}=9.5440(1)$ & $\beta=111.371(1)^{\circ}$ & $\mathrm{b}=10.1322(2)$ & $\beta=106.521(2)^{\circ}$ & $\mathrm{b}=9.8895(2)$ & $\beta=105.569(2)^{\circ}$ \\
\hline & $c=19.5581(3)$ & $\gamma=90^{\circ}$ & $c=18.5936(4)$ & $\gamma=90^{\circ}$ & $c=17.0426(4)$ & $\nu=90.183(2)^{\circ}$ \\
\hline Volume & \multicolumn{2}{|r|}{$2800.66(7) \AA^{3}$} & \multicolumn{2}{|r|}{$1482.39(6) \AA^{3}$} & \multicolumn{2}{|c|}{$2670.95(10) \AA^{3}$} \\
\hline$Z$ & \multicolumn{2}{|r|}{4} & \multicolumn{2}{|r|}{2} & \multicolumn{2}{|r|}{4} \\
\hline Density (calculated) & & $1.173 \mathrm{~g} \cdot \mathrm{cm}^{-3}$ & & $1.153 \mathrm{~g} . \mathrm{cm}^{-3}$ & \multicolumn{2}{|r|}{$1.161 \mathrm{~g} \cdot \mathrm{cm}^{-3}$} \\
\hline Absorption coefficient & & $0.152 \mathrm{~mm}^{-1}$ & & $0.144 \mathrm{~mm}^{-1}$ & \multicolumn{2}{|r|}{$0.108 \mathrm{~mm}^{-1}$} \\
\hline$F(000)$ & & 1056 & & 548 & \multicolumn{2}{|r|}{992} \\
\hline Crystal size & $0.2 x$ & $0.15 \times 0.1 \mathrm{~mm}^{3}$ & 0.8 & $\times 0.5 \times 0.4 \mathrm{~mm}^{3}$ & \multicolumn{2}{|c|}{$0.3 \times 0.2 \times 0.1 \mathrm{~mm}^{3}$} \\
\hline Orange for data collection & & $.357^{\circ}$ to $30.541^{\circ}$ & & $247^{\circ}$ to $30.587^{\circ}$ & \multicolumn{2}{|c|}{$1.436^{\circ}$ to $30.580^{\circ}$} \\
\hline Index ranges & $-23<=\mathrm{h}<=2$ & $\begin{aligned} & 2,-13<=k<=13,- \\
& 27<=\mid<=27\end{aligned}$ & $-13<=h<=1$ & $\begin{array}{r}3,-14<=k<=14,- \\
24<=k<=24\end{array}$ & \multicolumn{2}{|c|}{$\begin{array}{r}-21<=\mathrm{h}<=21,-14<=\mathrm{k}<=14,- \\
26<=\mathrm{k}<=26\end{array}$} \\
\hline Reflections collected & & 34571 & & 35861 & \multicolumn{2}{|r|}{45252} \\
\hline Independent reflections & 8533 & {$[R($ int $)=0.0213]$} & 9053 & {$[R($ int $)=0.0137]$} & \multicolumn{2}{|c|}{$8193[R($ int $)=0.0198]$} \\
\hline Completeness to $\theta=25.242^{\circ}$ & & $100.0 \%$ & & $99.6 \%$ & \multicolumn{2}{|r|}{$100.0 \%$} \\
\hline Absorption correction & Semi & $\begin{array}{r}\text { i-empirical from } \\
\text { equivalents }\end{array}$ & Sem & $\begin{array}{r}\text { i-empirical from } \\
\text { equivalents }\end{array}$ & \multicolumn{2}{|c|}{$\begin{array}{r}\text { Semi-empirical from } \\
\text { equivalents }\end{array}$} \\
\hline Max. and min. transmission & & 0.746 and 0.720 & & 0.746 and 0.721 & \multicolumn{2}{|r|}{0.746 and 0.723} \\
\hline Refinement method & Full-matrix lea & st-squares on $\mathrm{F}^{2}$ & Full-matrix lea & st-squares on $\mathrm{F}^{2}$ & \multicolumn{2}{|c|}{ Full-matrix least-squares on $\mathrm{F}^{2}$} \\
\hline $\begin{array}{l}\text { Data / restraints / } \\
\text { parameters }\end{array}$ & & 8533 / 0 / 316 & & 9053 / 0 / 334 & \multicolumn{2}{|r|}{$8193 / 0 / 318$} \\
\hline Goodness-of-fit on $F^{2}$ & & 1.032 & & 1.040 & & 1.047 \\
\hline Final $R$ indices $[I>2 \sigma(I)]$ & $\mathrm{R} 1=0.038$ & $9, w R 2=0.1088$ & $\mathrm{R} 1=0.043$ & $7, w R 2=0.1090$ & \multicolumn{2}{|c|}{$\mathrm{R} 1=0.0416, w R 2=0.1075$} \\
\hline$R$ indices (all data) & $\mathrm{R} 1=0.053$ & $9, w R 2=0.1248$ & $\mathrm{R} 1=0.049$ & $4, w R 2=0.1154$ & \multicolumn{2}{|c|}{$\mathrm{R} 1=0.0553, w R 2=0.1193$} \\
\hline $\begin{array}{l}\text { Largest difference peak and } \\
\text { hole }\end{array}$ & \multicolumn{2}{|c|}{0.356 and -0.214 e. $\AA^{-3}$} & \multicolumn{2}{|c|}{0.427 and -0.225 e. $\AA^{-3}$} & \multicolumn{2}{|c|}{0.340 and -0.191 e. $\AA^{-3}$} \\
\hline
\end{tabular}




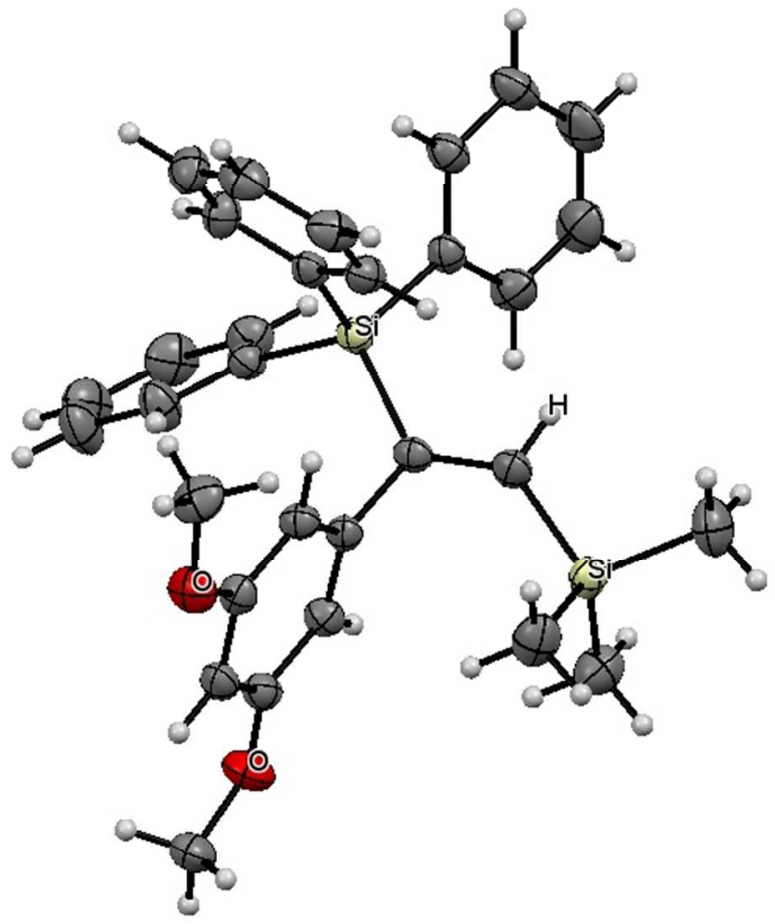

Figure S4. ORTEP view of compound 3ha.

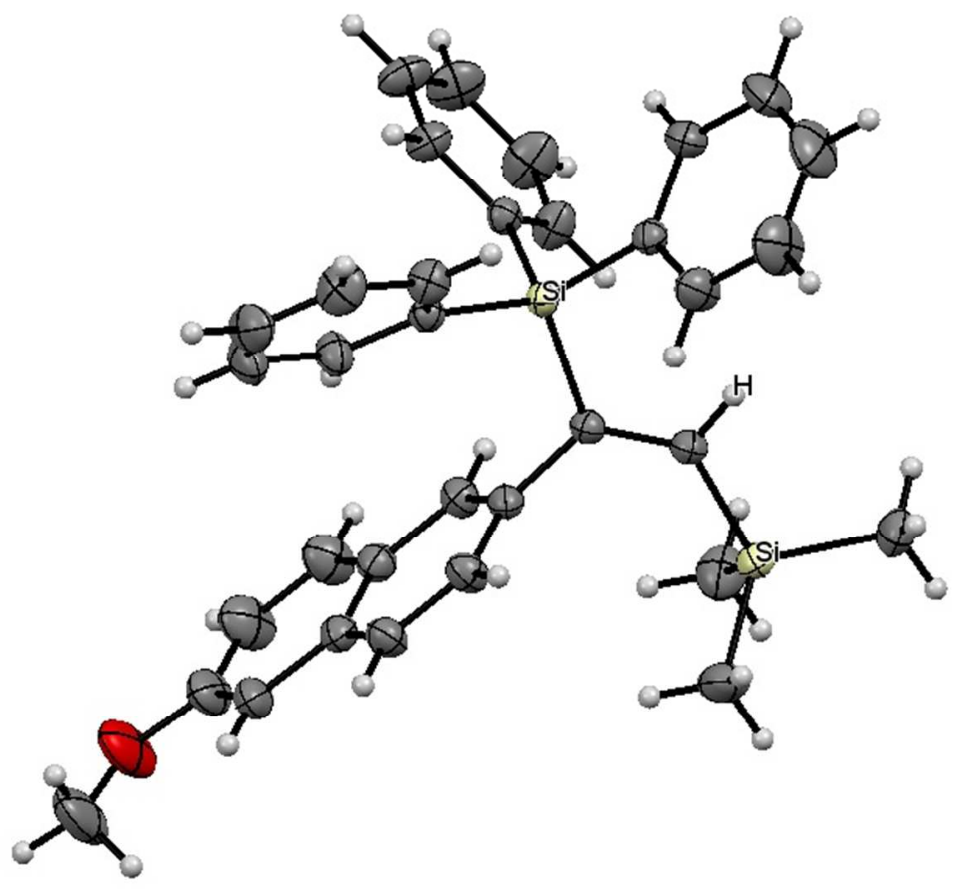

Figure S5. ORTEP view of compound 3ia. 


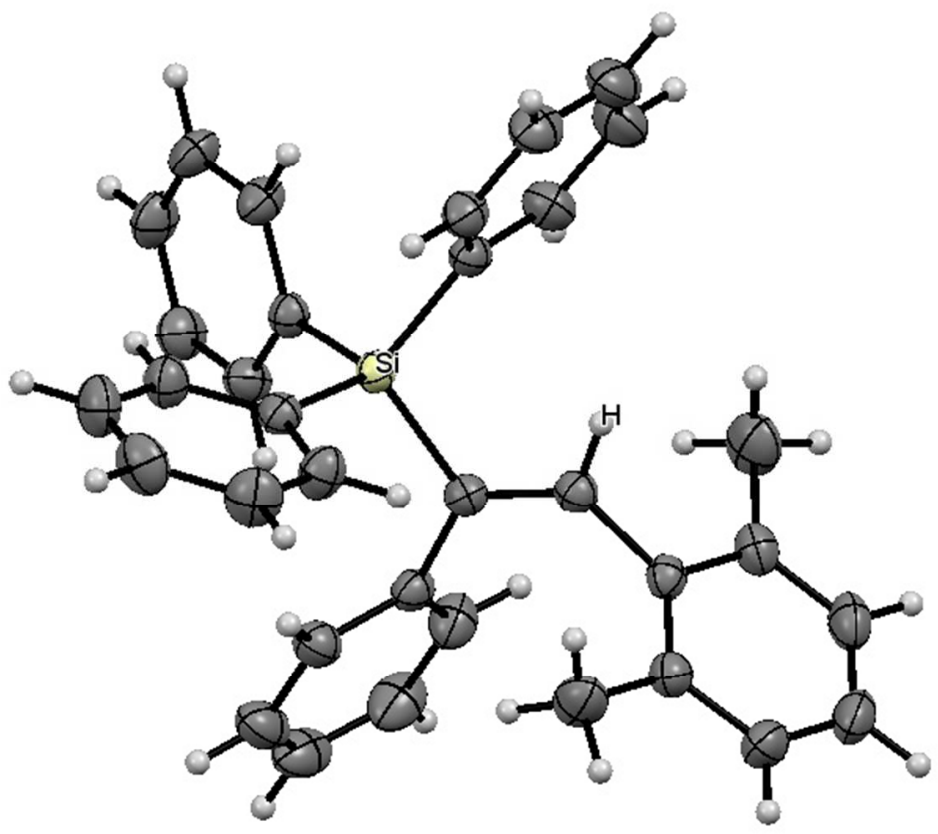

Figure S6. ORTEP view of compound 3la. 


\section{References}

(1) Perrin, D. D.; Armarego, W. L. F. Purification of Laboratory Chemicals; 3rd ed., Pergamon Press, Oxford, 1988.

(2) Still, W. C.; Kahn, M.; Mitra, A. J. J. Org. Chem. 1978, 43, 2923.

(3) Cano, R.; Yus, M.; Ramón, D. J. ACS Catal. 2012, 2, 1070.

(4) Green, M.; Spencer, J. L.; Stone, F.; Gordon, A.; Tsipis, C. A. J. Chem. Soc. Dalton Trans. 1977, 1525.

(5) Planellas, M.; Guo, W.; Alonso, F.; Yus, M.; Shafir, A.; Pleixats, R.; Parella, T. Adv. Synth. Catal. 2014, 356, 179.

(6) Brook, A. G.; Pannell, K. H.; Anderson, D. G. J. Am. Chem. Soc. 1968, 90, 4374.

(7) Tillack, A.; Pulst, S.; Baumann, W.; Baudisch, H.; Kortus, K.; Rosenthal, U. J. Organomet. Chem. 1997, 532, 117.

(8) Prepared according to the procedure described in Wrackmeyer, B.; Milius, W.; Tok, O.L. Chem. Eur. J. 2003, 9, 4732.

(9) Prepared according to the procedure described in Cao, D.; Kolshorn, H.; Meier, H. Tetrahedron Lett. 1996, 37, 4487.

(10) A. G. Brook, J. M. Duff, and W. F. Reynolds, J. Organometallic Chem., 1976, 121, 293

(11) Psyllaki, A.; Lykakis, I. N.: Stratakis M. Tetrahedron 2012, 68, 8724.

(12) a) Sanada, T.; Kato, T.; Mitani, M.; Mori, A. Adv. Synth. Catal. 2006, 348, 51. b) Chauhan, M.; Hauck, B. J.; Keller, L. P.; Boudjouk, P. J. Organomet. Chem. 2002, 645, 1.

(13) Blessing, R. H. Acta Cryst. A 1995, 51, 33-38.

(14) Sheldrick, G. M. Acta Cryst. A 2008, 64, 112-122.

(15) Farrugia, L.J. J. Appl. Cryst. 1999, 32, 837-838. 


\section{Triphenyl-[(E)-(1,2-diphenylvinyl)]silane (3aa)}

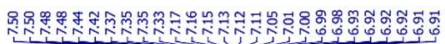<smiles>C(=C(c1ccccc1)c1ccccc1)c1ccccc1</smiles>
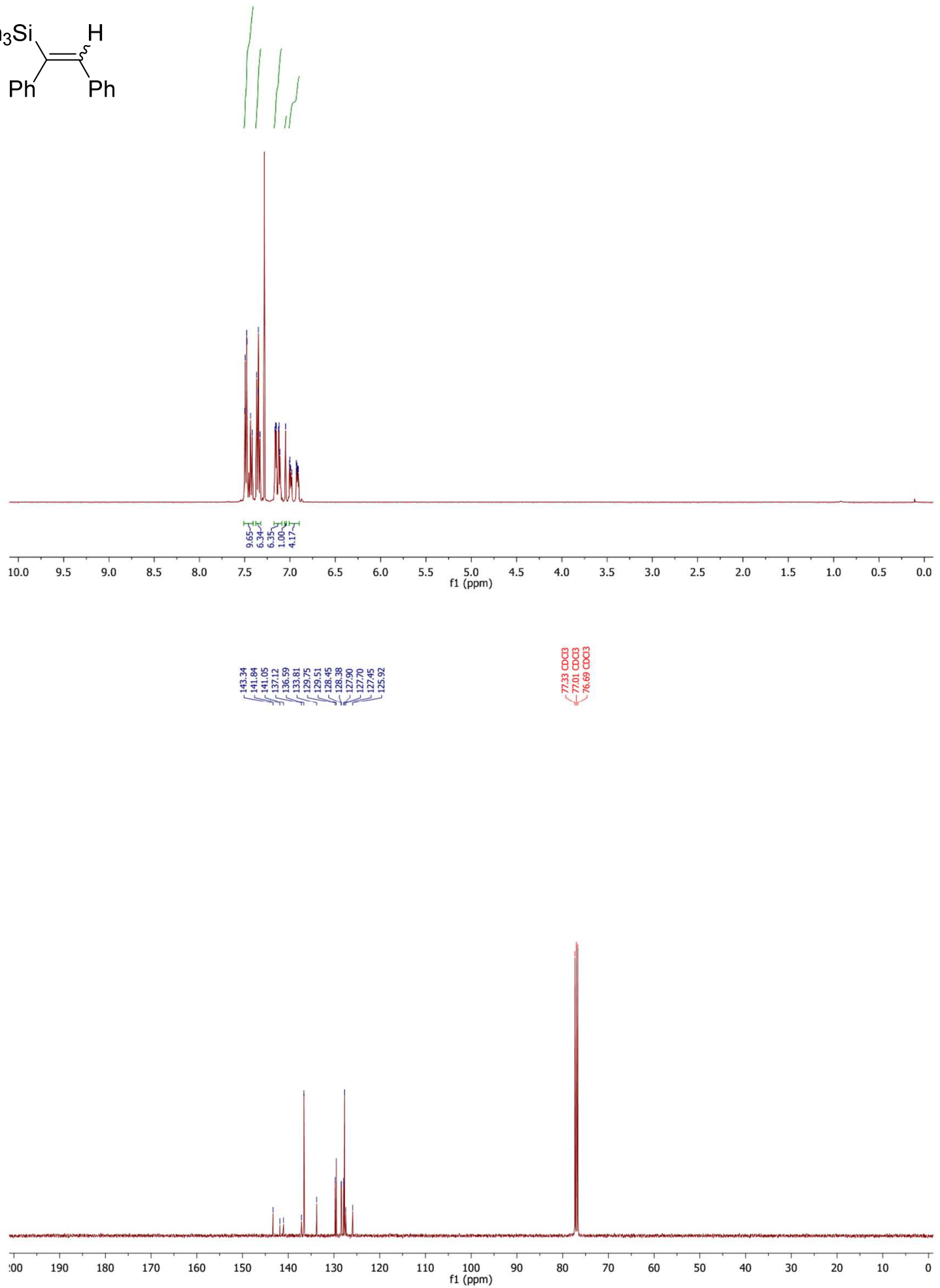
Triethoxy-[(E)-(1,2-diphenylvinyl)]silane (3ab)

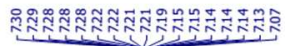

:

$(\mathrm{EtO})_{3} \mathrm{Si} \underbrace{\mathrm{H}}_{\mathrm{Ph}}$
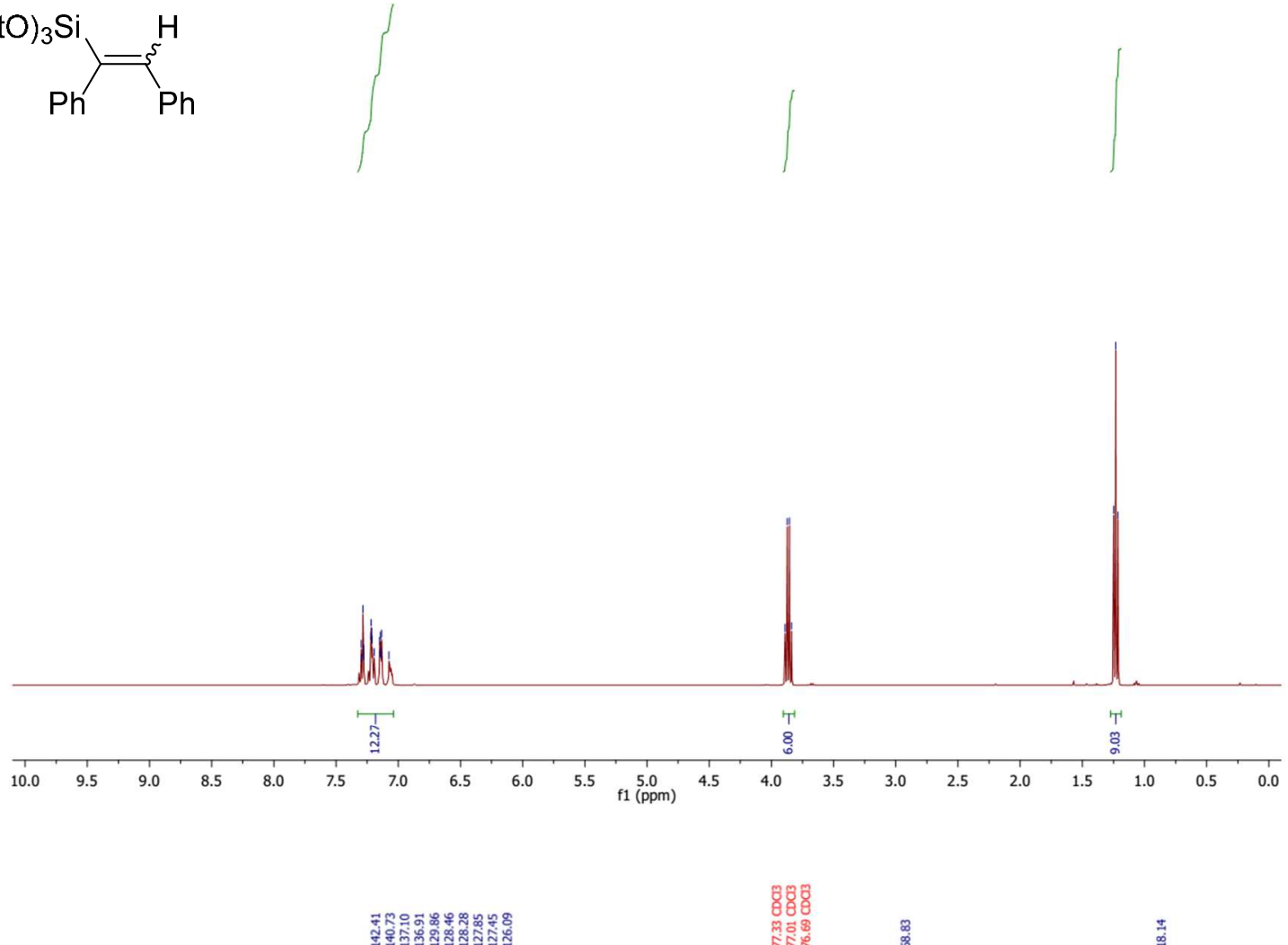

1.
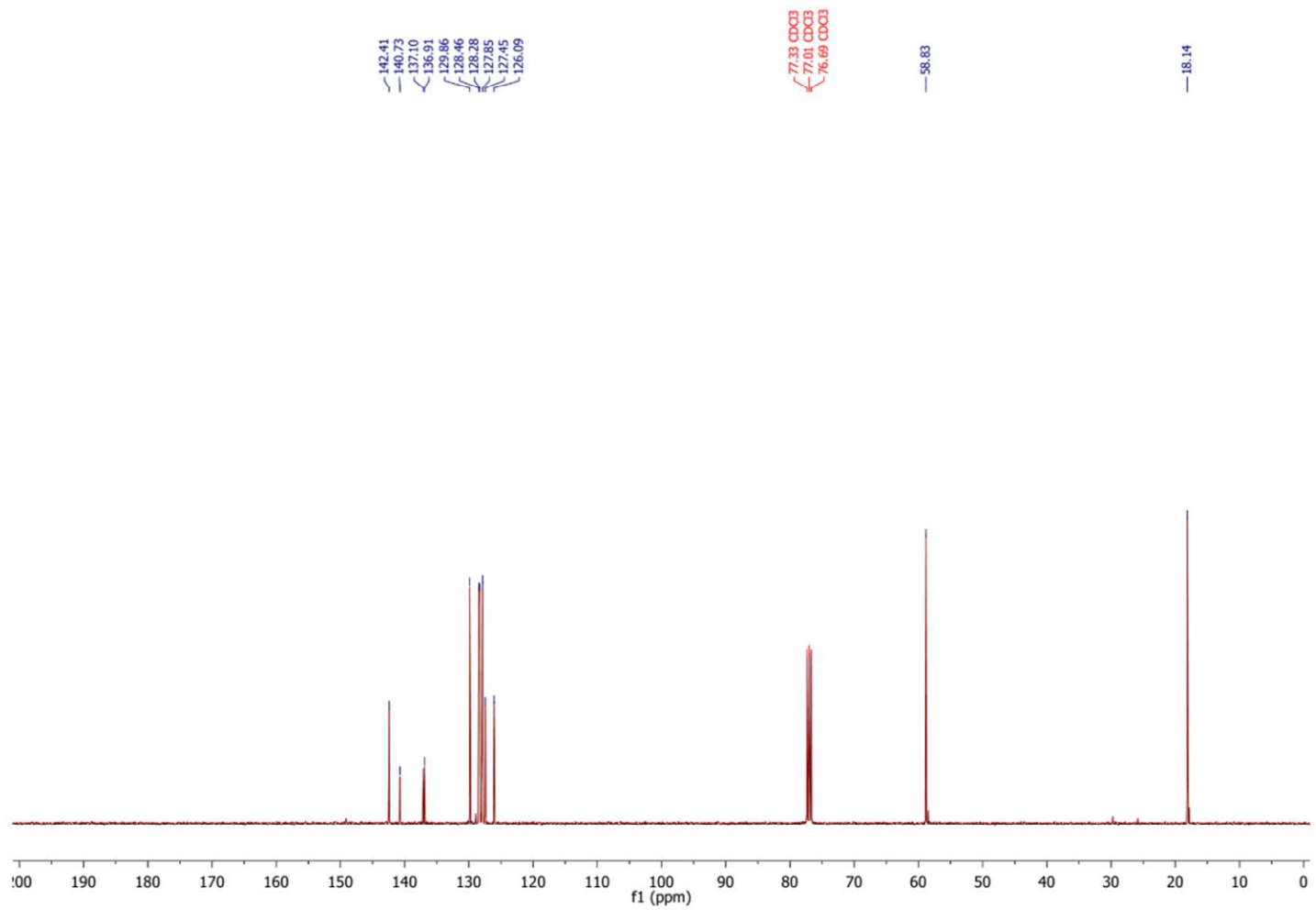
Triethyl-[(E)-(1,2-diphenylvinyl)]silane (3ac)

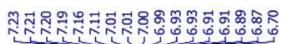

$\mathrm{Et}_{3} \mathrm{Si} \underbrace{\mathrm{H}}_{\mathrm{Ph}}$
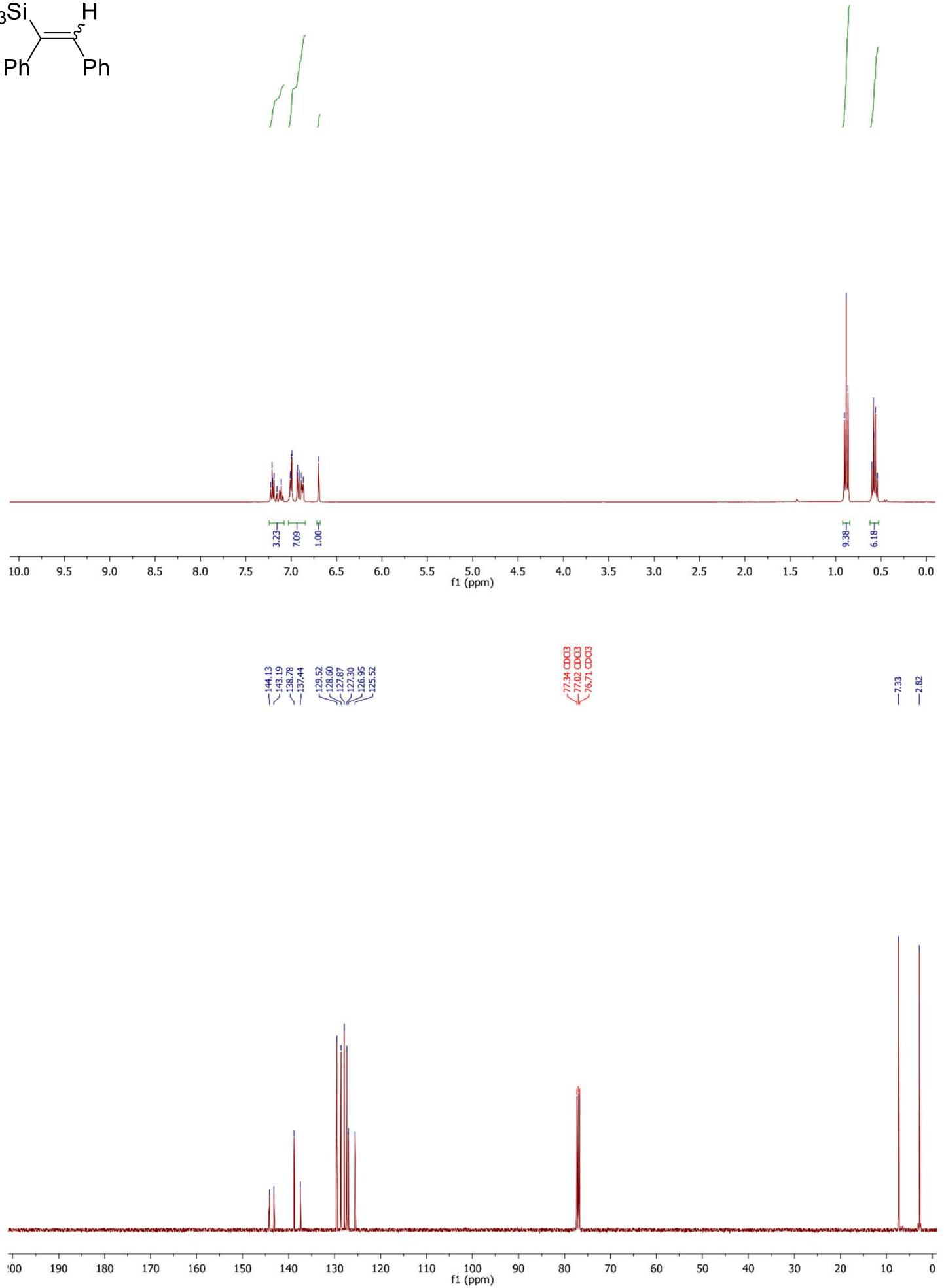
Dimethylphenyl-[(E)-(1,2-diphenylvinyl)]silane (3ad)

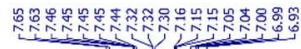

$\mathrm{Me}_{2} \mathrm{PhSi} \underbrace{\mathrm{H}}_{\mathrm{Ph}}$
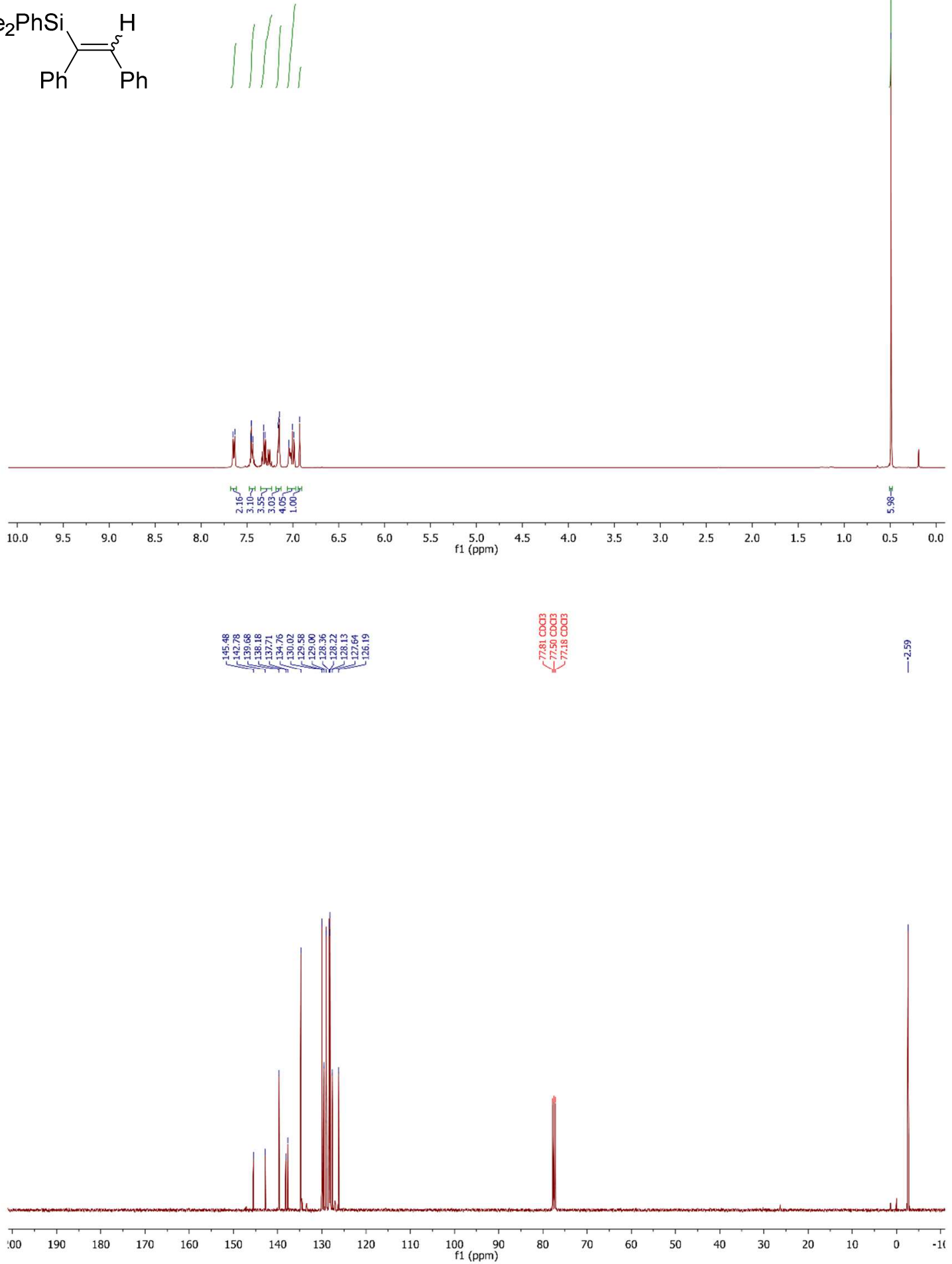
Methyldiphenyl-[(E)-(1,2-diphenylvinyl)]silane (3ae)

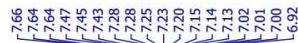<smiles>CC(=O)c1ccccc1</smiles>
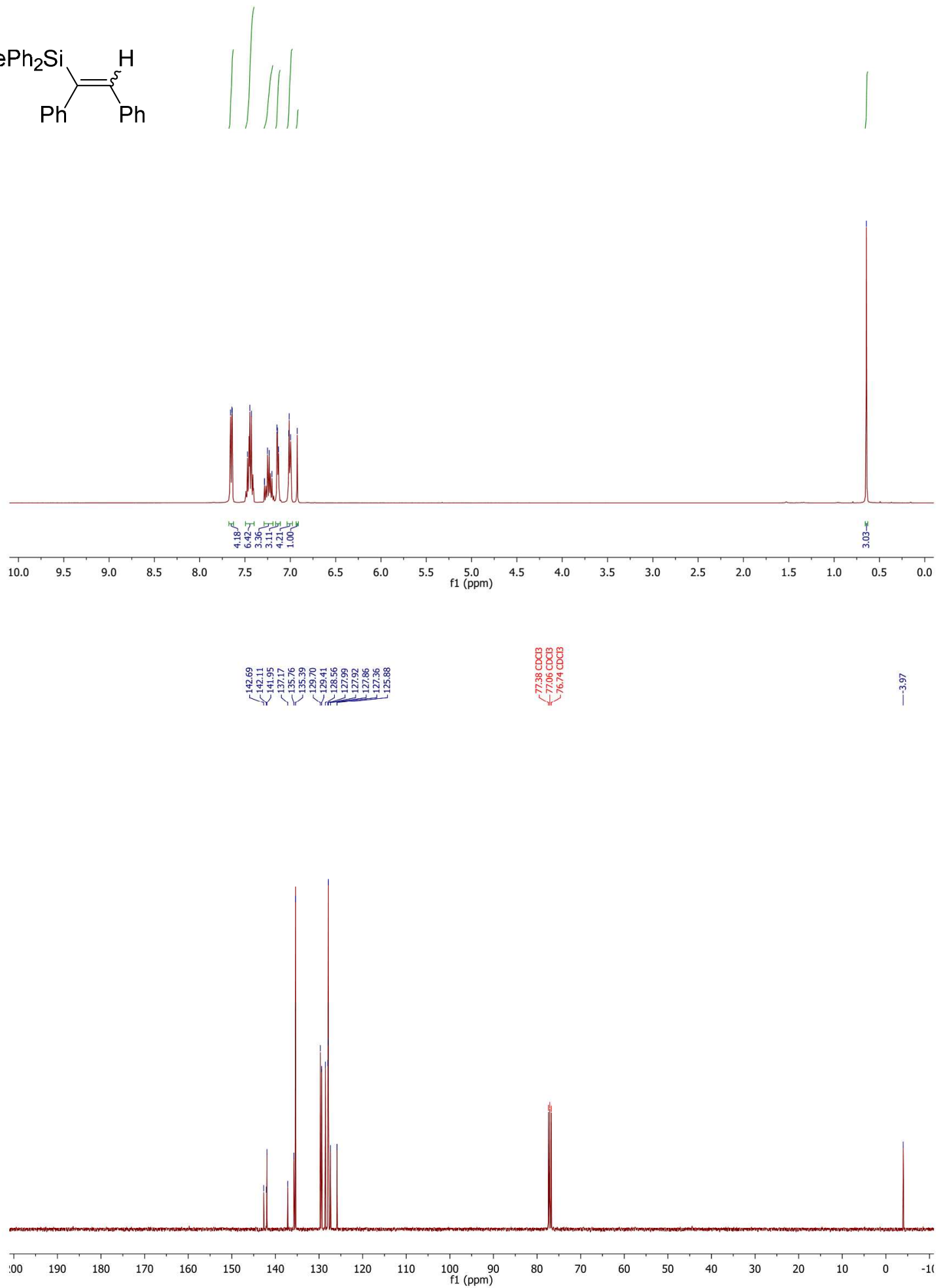
Methyldiethoxy-[(E)-(1,2-diphenylvinyl)]silane (3af)
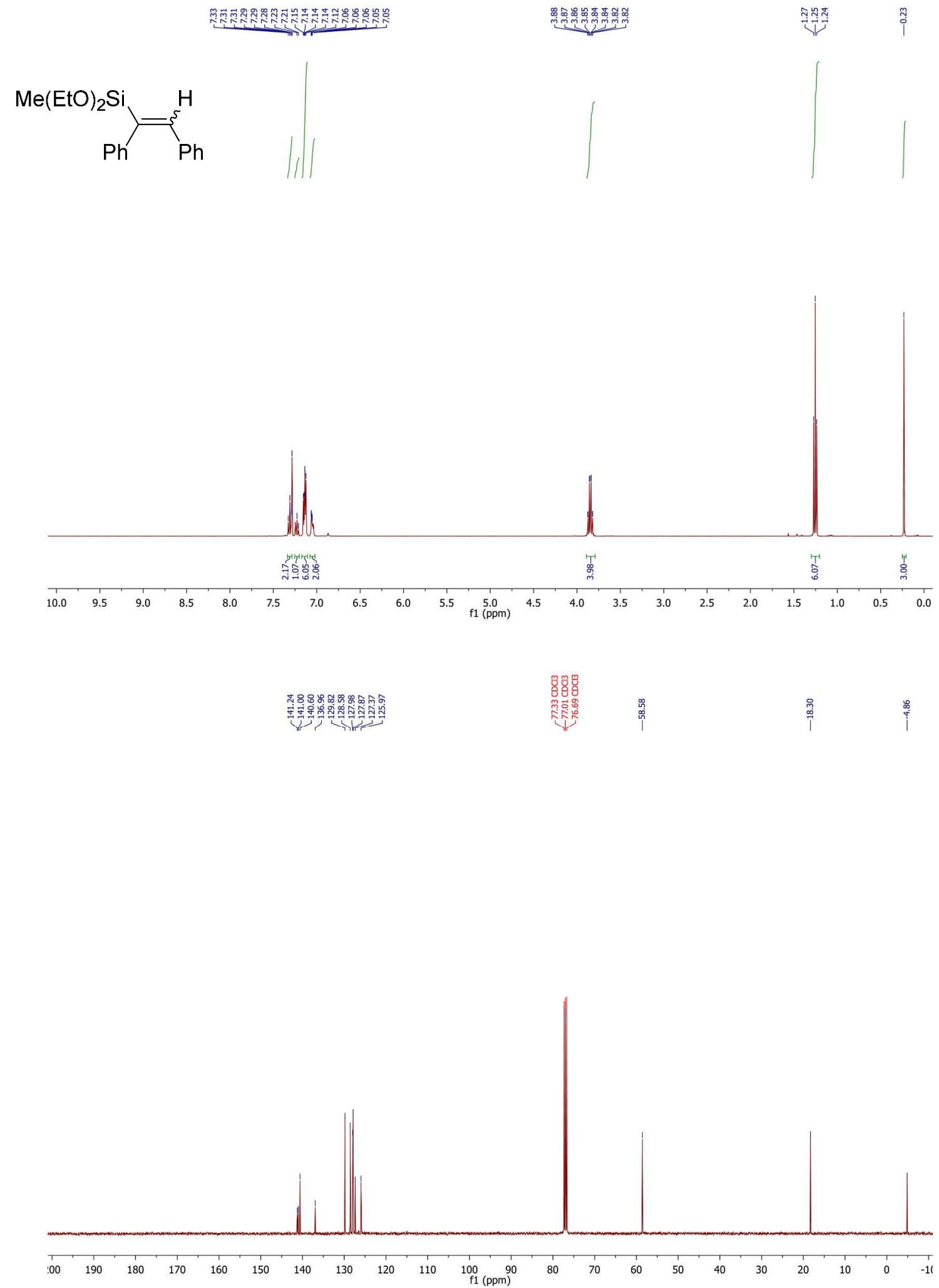
Diisopropyl[(trimethylsilyl)ethynyl][(E)-(1,2-diphenylvinyl)]silane (3ag)
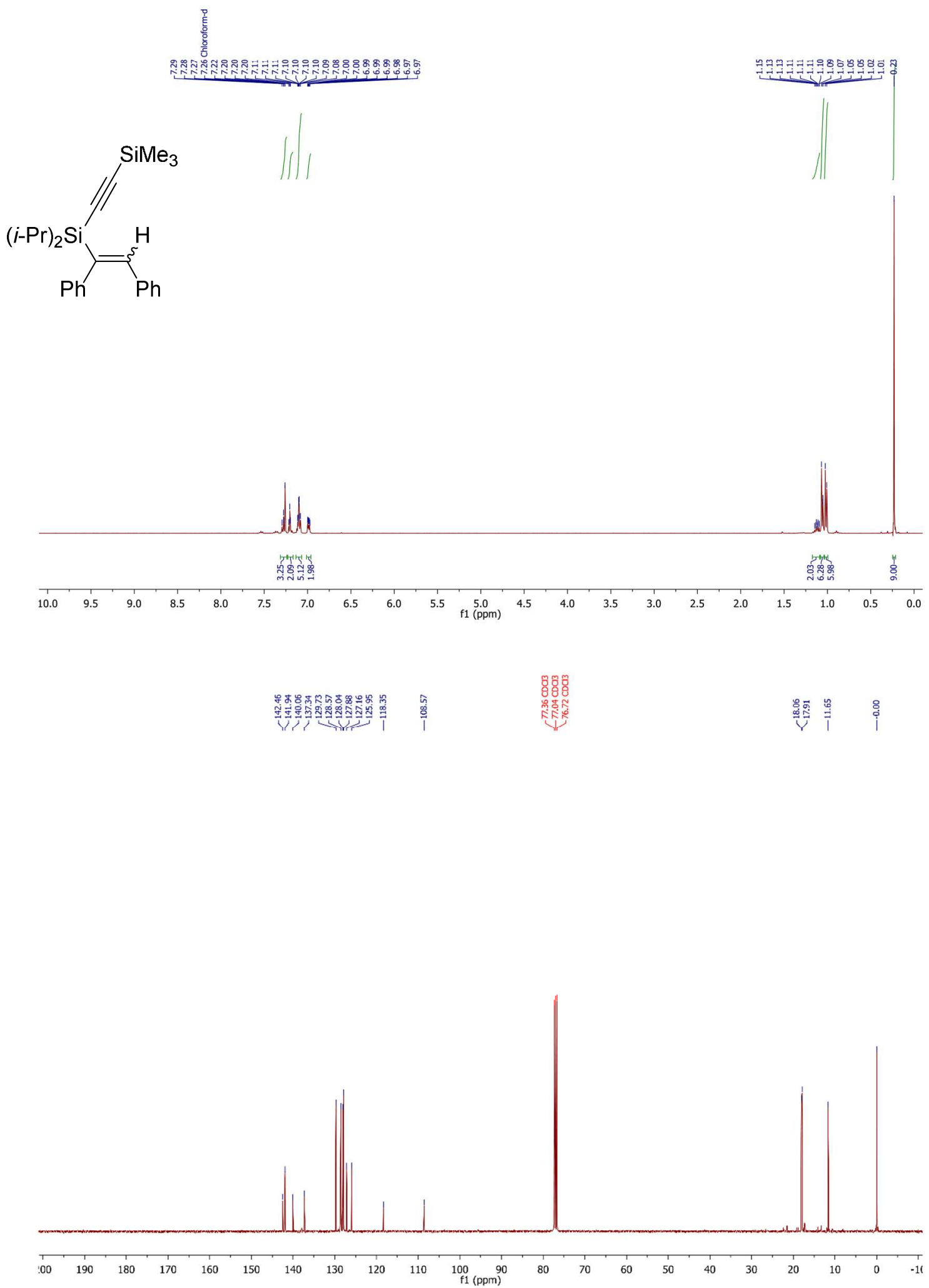
Triphenyl-[(E)-(1-phenyl-2-butylvinyl)]silane (3ba)

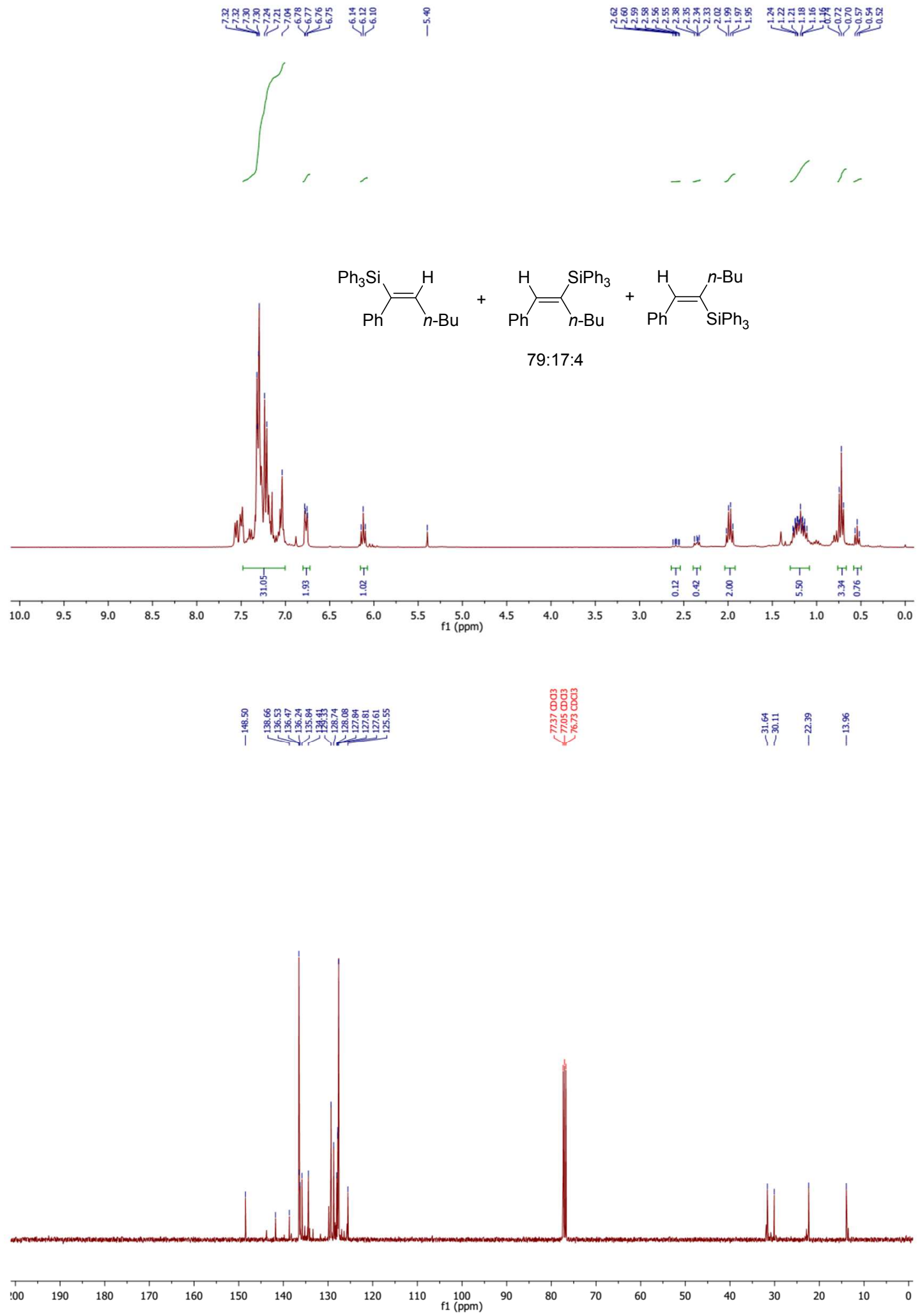


Triphenyl-[(E)-(1-(4-methoxyphenyl)-2-butylvinyl)]silane (3ca)

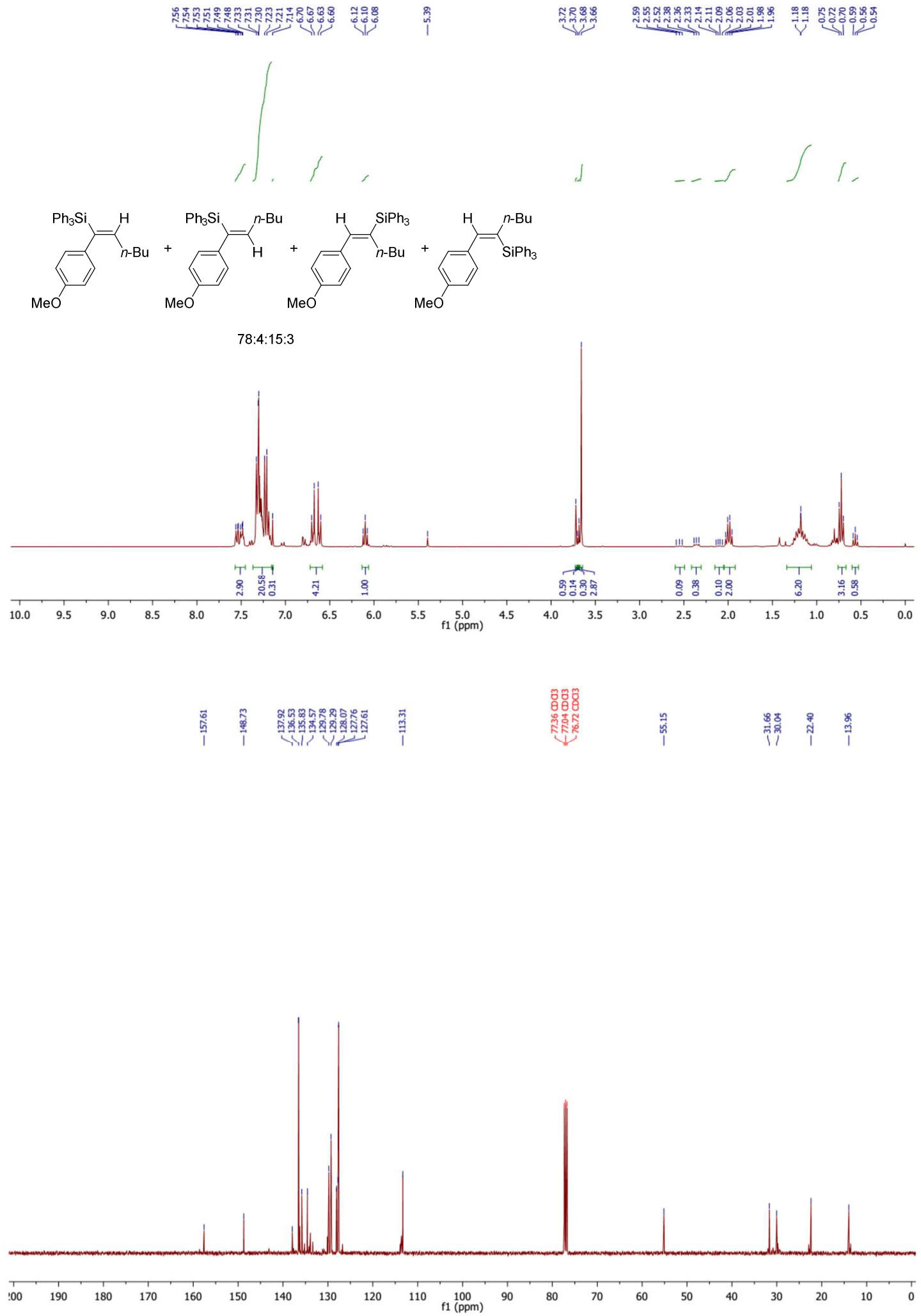


Triphenyl-[(E)-(1-(4-(trifluoromethyl)phenyl)-2-butylvinyl)]silane (3da)
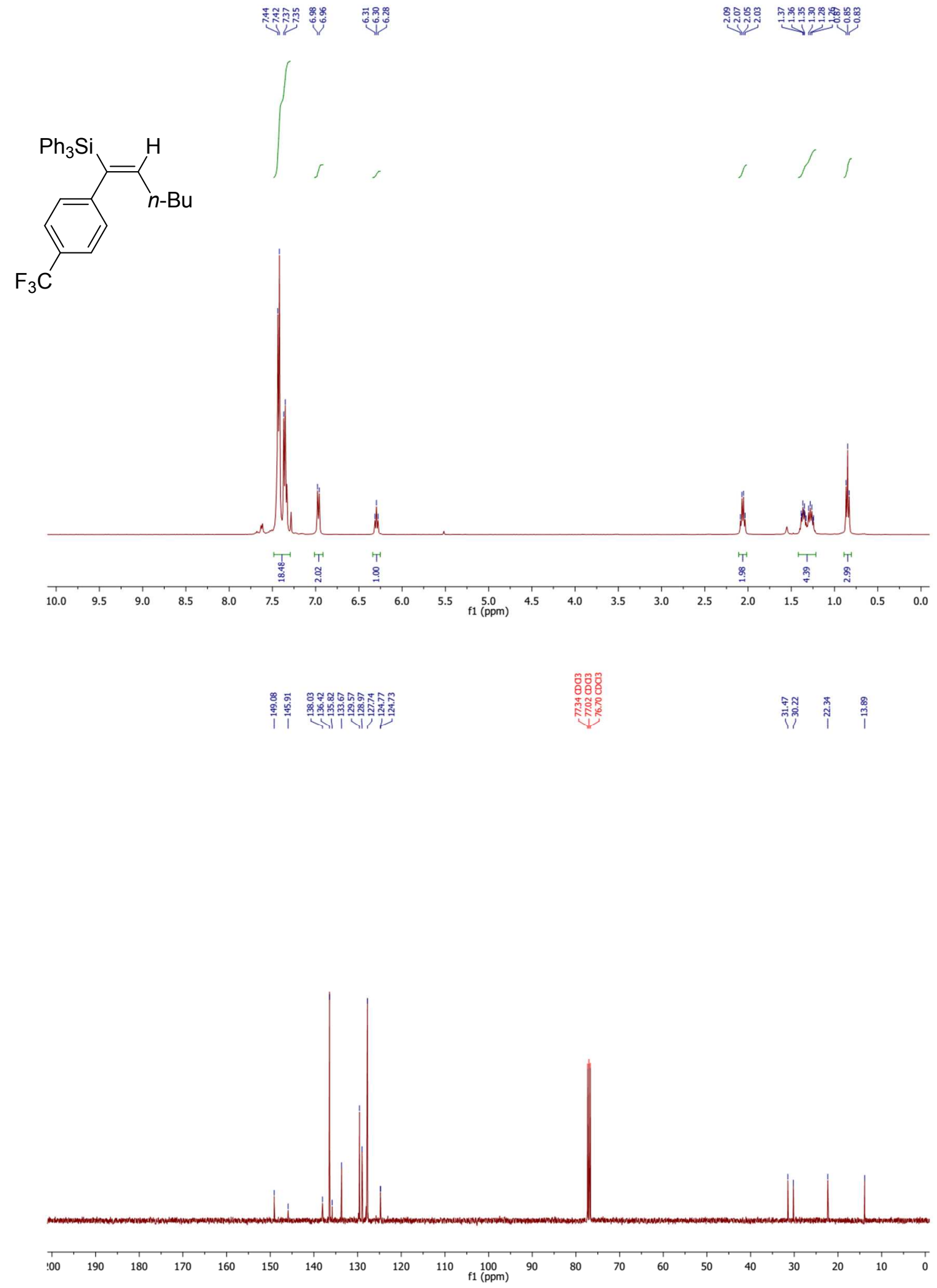
Triphenyl-[(E)-(1-phenyl-2-(trimethylsilyl)vinyl)]silane (3ea)

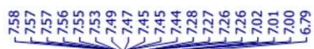

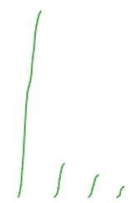

${ }_{\mathrm{Ph}}^{\mathrm{Ph}_{3} \mathrm{Si}}=\mathrm{TMS}_{\mathrm{TMS}}^{\mathrm{H}}$
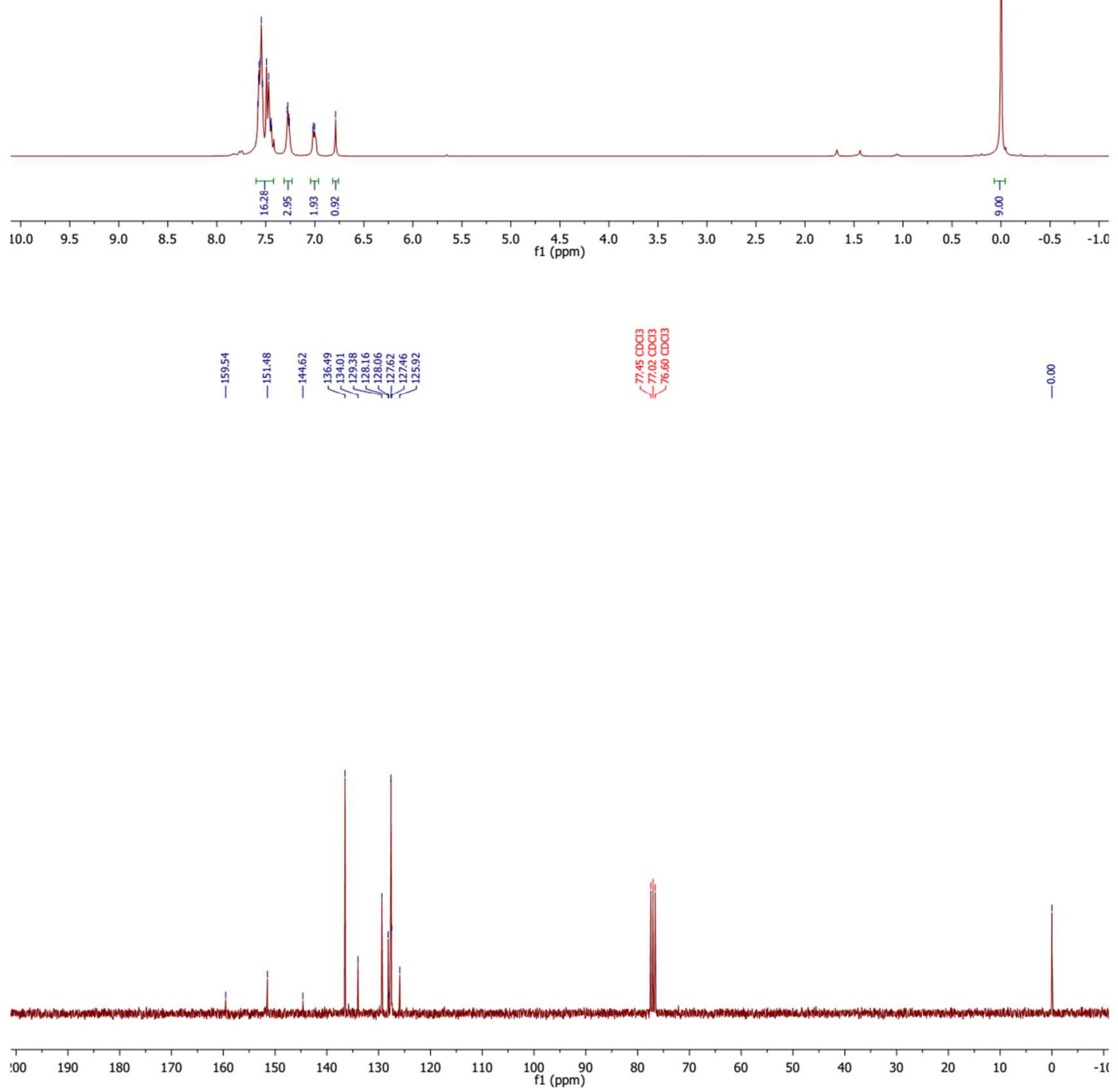
Triphenyl-[(E)-(1-(4-methoxyphenyl)-2-(trimethylsilyl)vinyl)]silane (3fa)
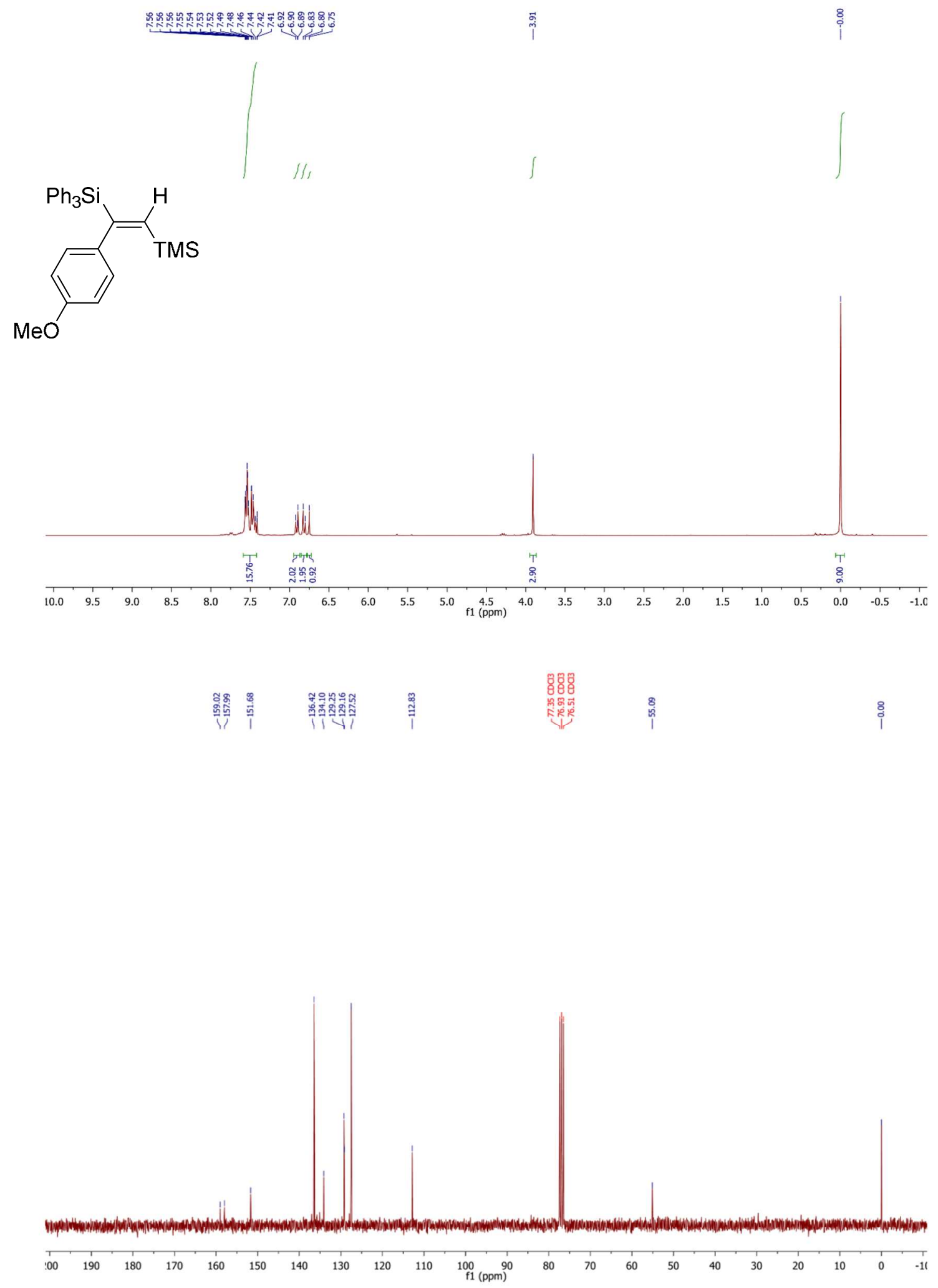
Triphenyl-[(E)-(1-(4-trifluoromethyl)phenyl-2-(trimethylsilyl)vinyl)]silane (3ga)
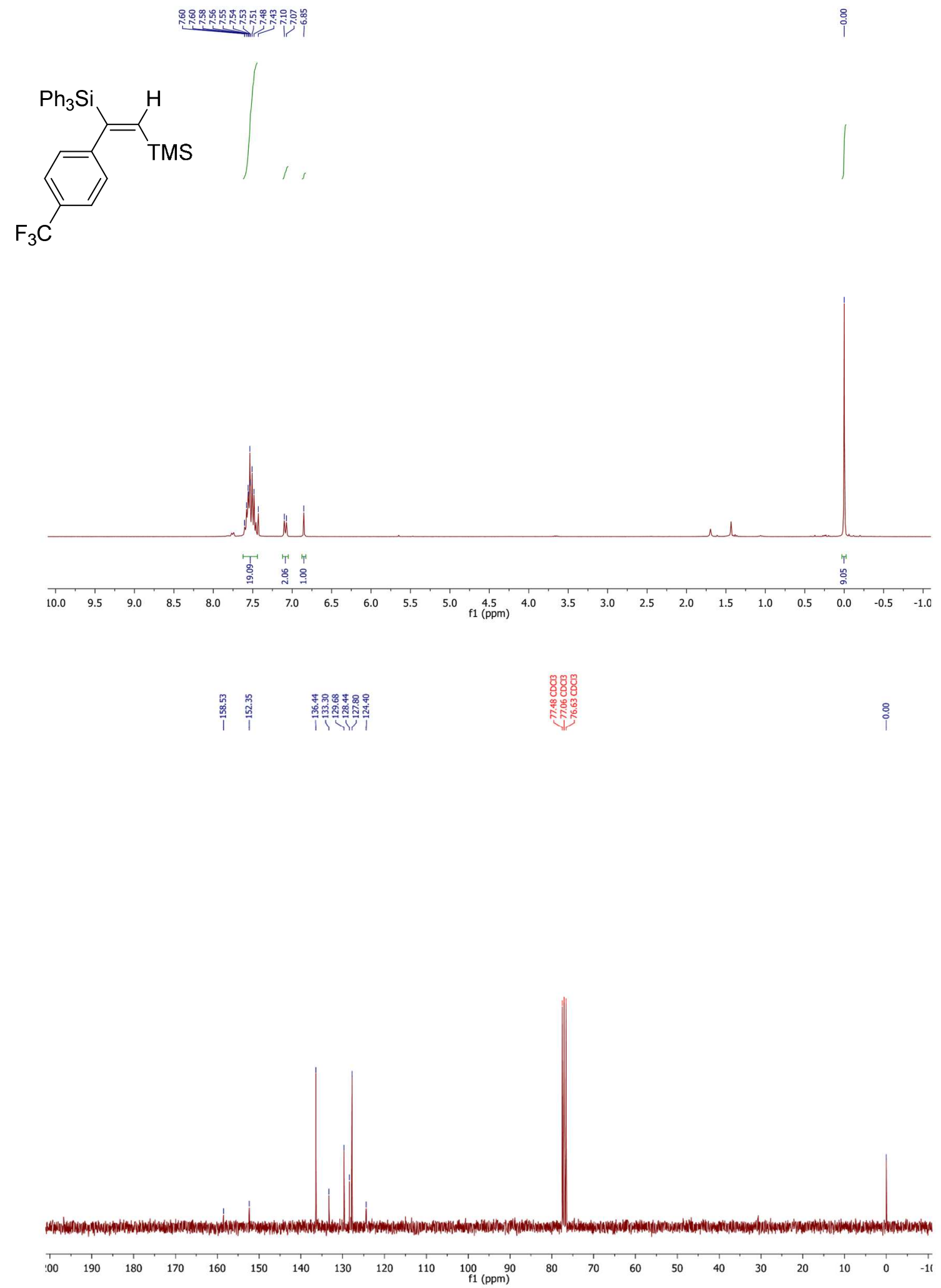
Triphenyl-[(E)-(1-(3,5-dimethoxyphenyl)-2-(trimethylsilyl)vinyl)]silane (3ha)
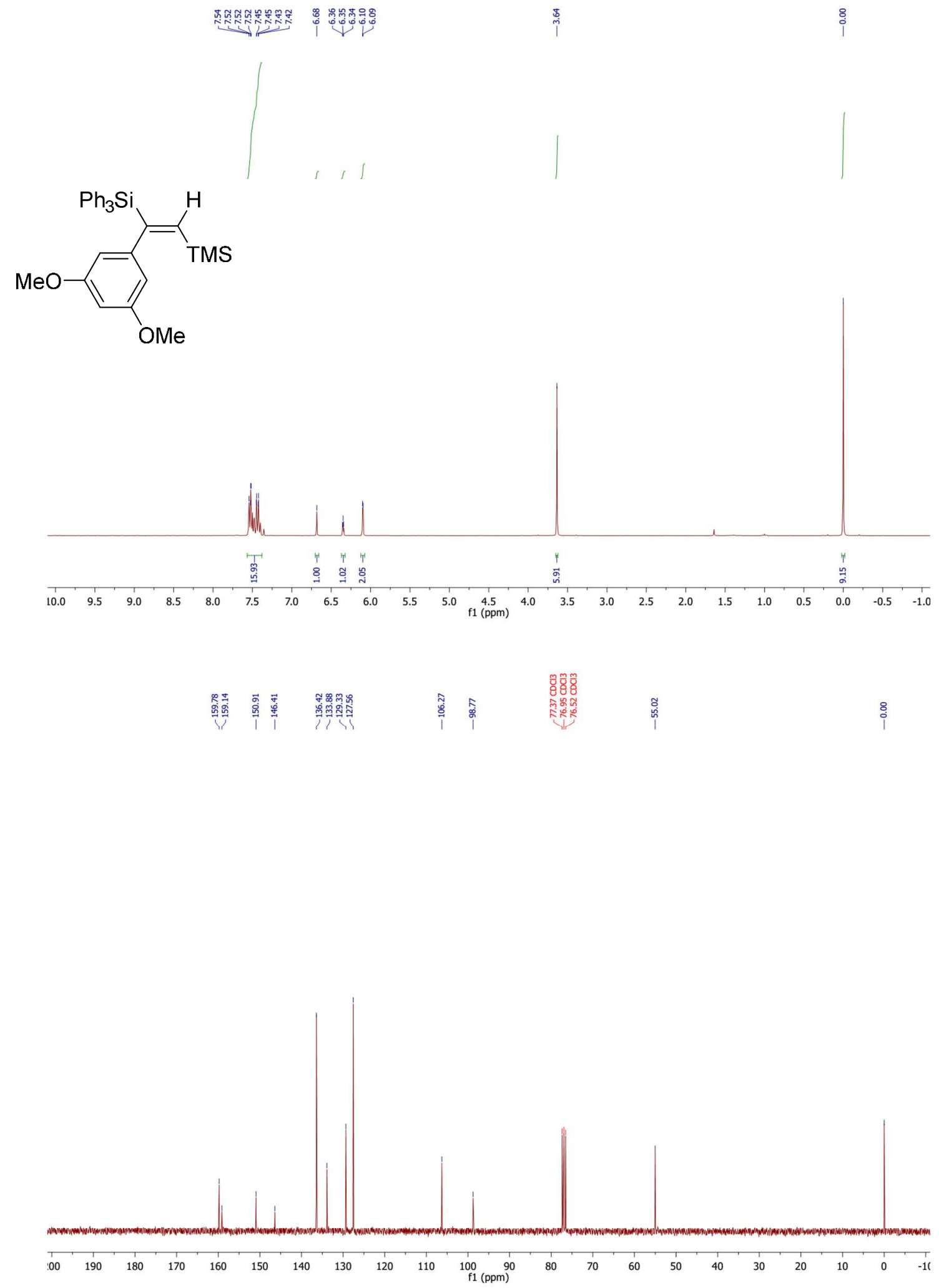
Triphenyl-[(E)-(1-(6-methoxynaphthalen-2-yl)-2-(trimethylsilyl)vinyl)]silane (3ia)

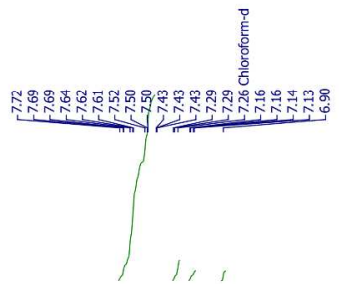<smiles>COc1ccc2cc(/C([SiH3])=C/[13CH3])ccc2c1</smiles>
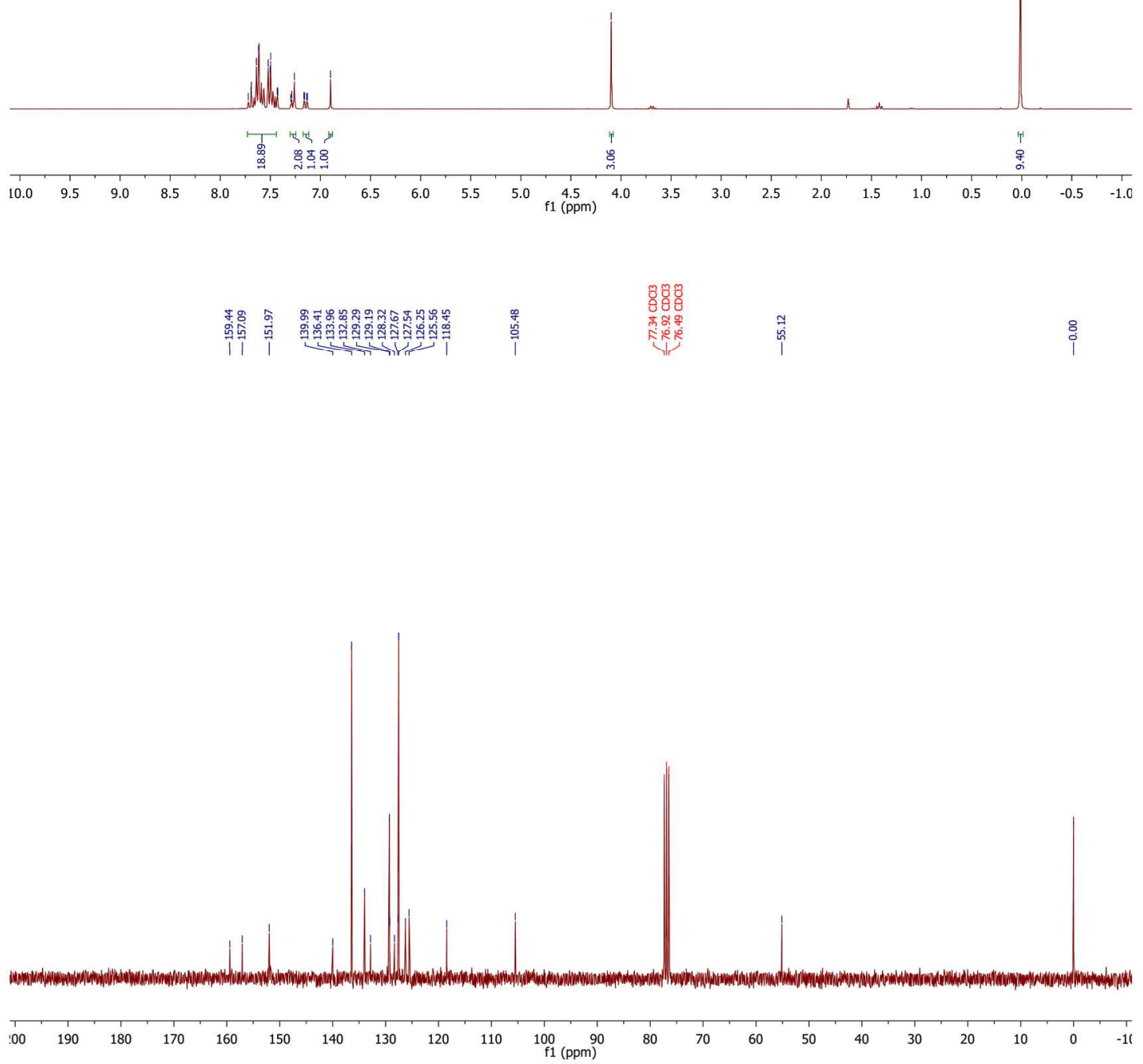
Triphenyl-[(E)-(1-(2-pyridyl)-2-(trimethylsilyl)vinyl)]silane (3ja)
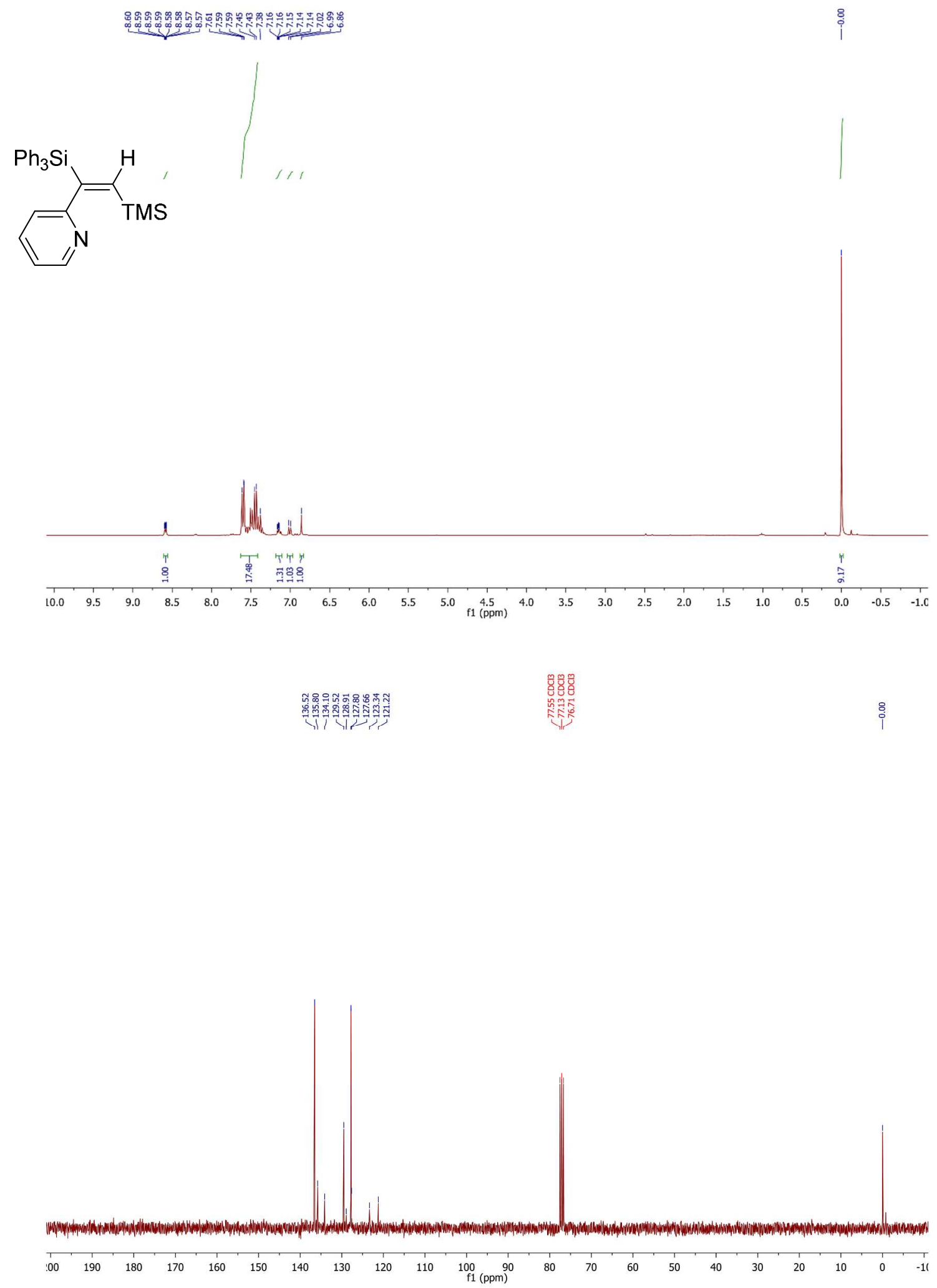
Triphenyl-[(E)-(1-(3-pyridyl)-2-(trimethylsilyl)vinyl)]silane (3ka)

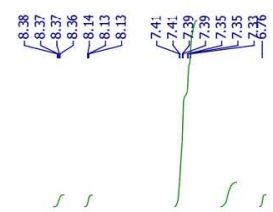<smiles>CC(=C([SiH2])C=S(C)(C)=O)c1cccnc1</smiles>
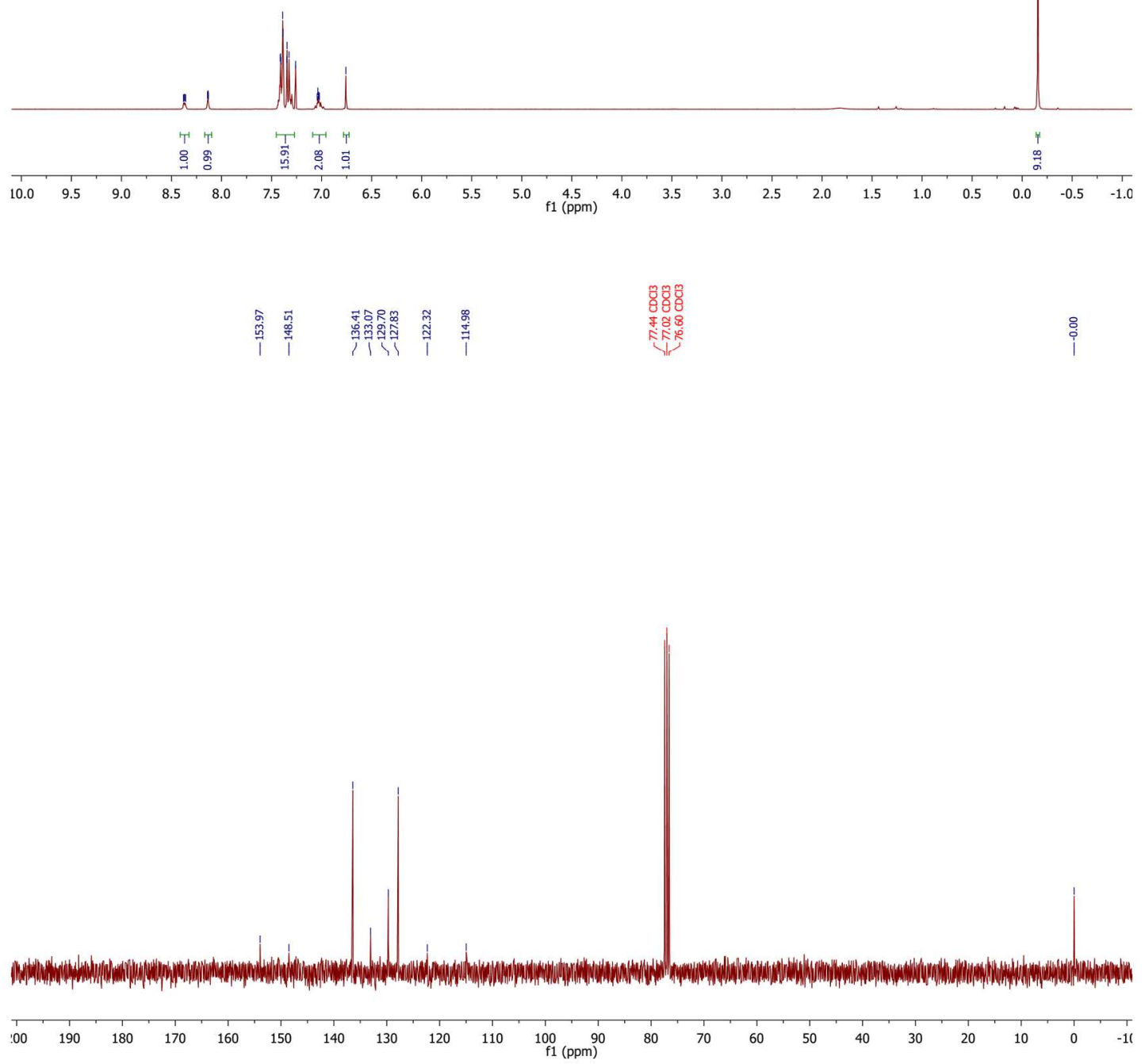
Triphenyl-[(E)-(1-phenyl-2-(2,6-dimethylphenyl)-vinyl)]silane (3la)
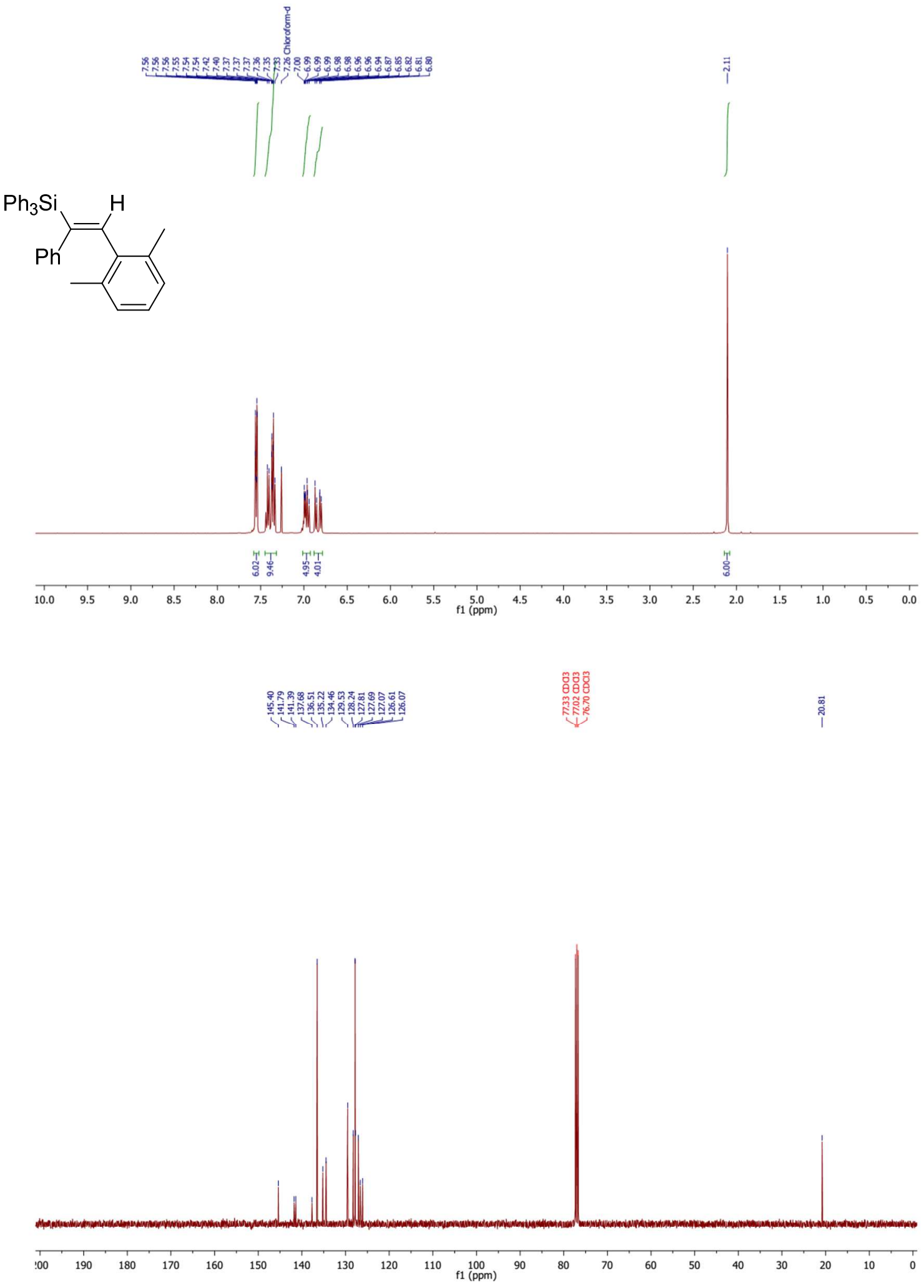
Triphenyl-[(E)-(1-phenylprop-1-en-1-yl)]silane (3ma)
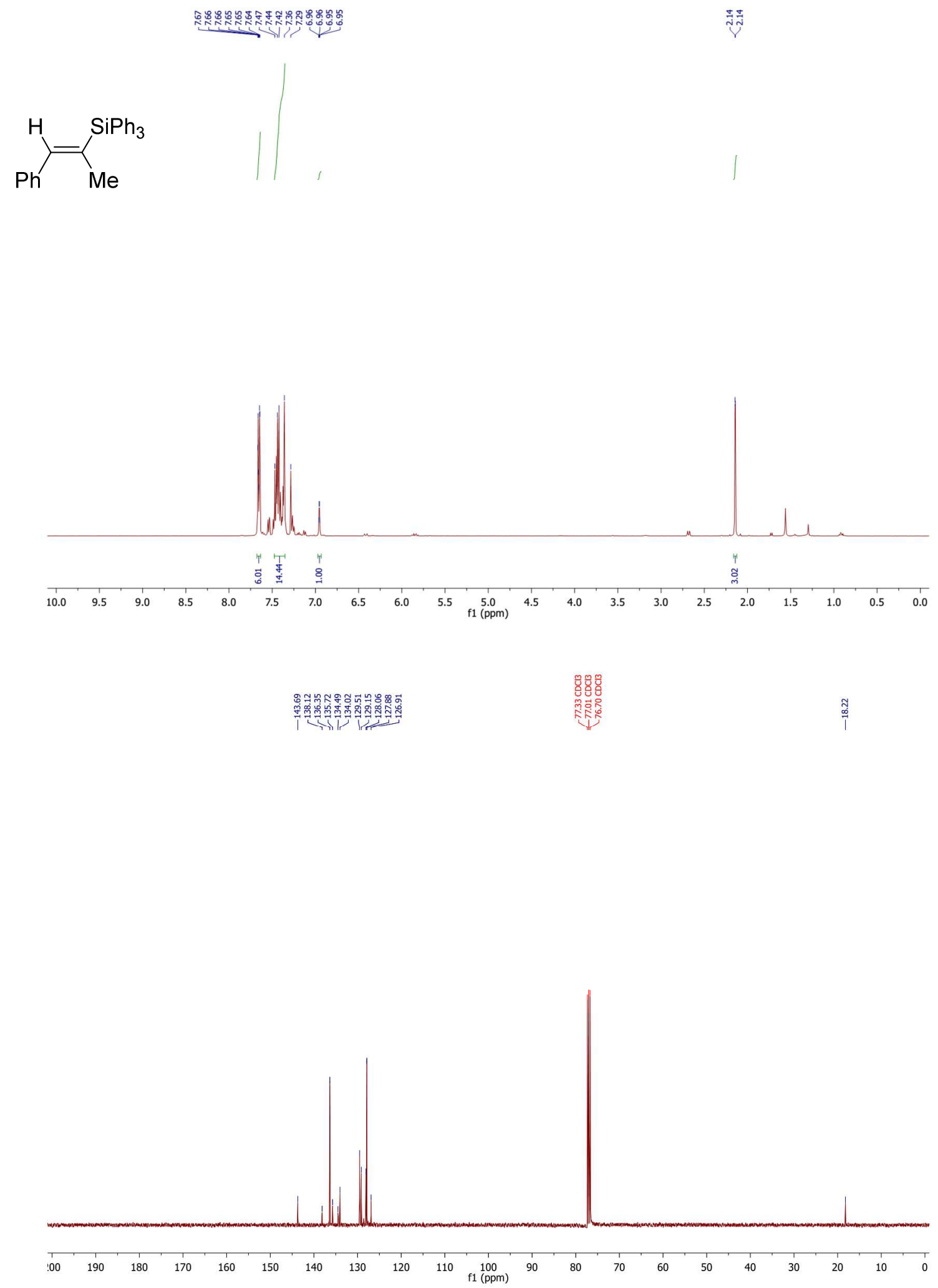
Triphenyl-[(E)-(1-(4-methoxyphenyl)-2-phenylvinyl)]silane and triphenyl-[(E)-(1-phenyl-2-(4methoxyphenyl)vinyl)]silane (3na)

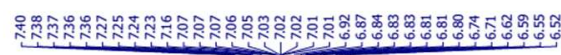

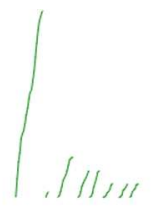

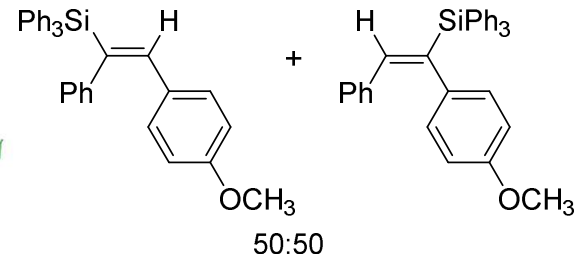
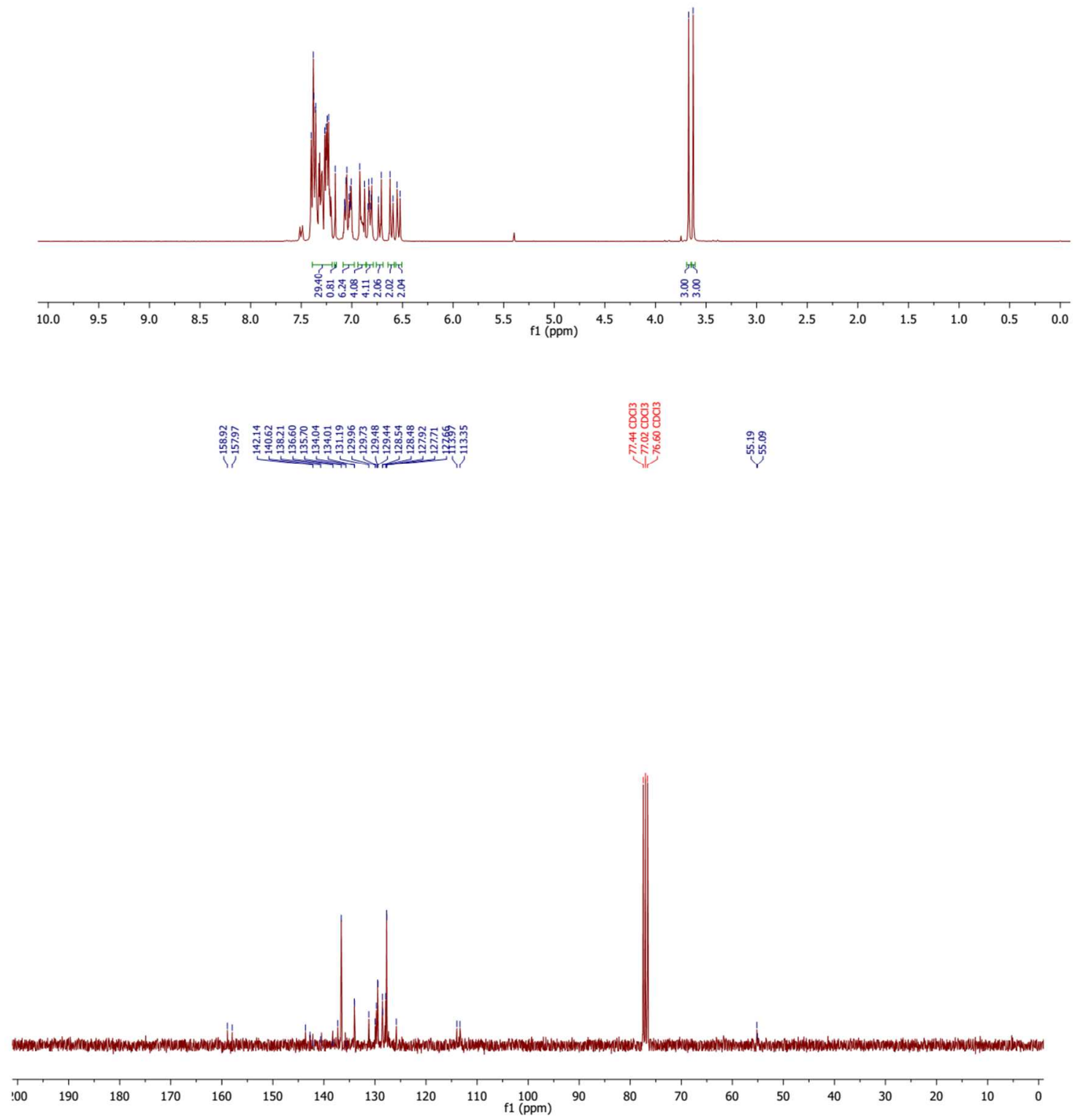
(E)-2-(2-phenyl-1-(triphenylsilyl)vinyl)pyridine and (E)-2-(2-phenyl-2-(triphenylsilyl)vinyl)pyridine (3oa)
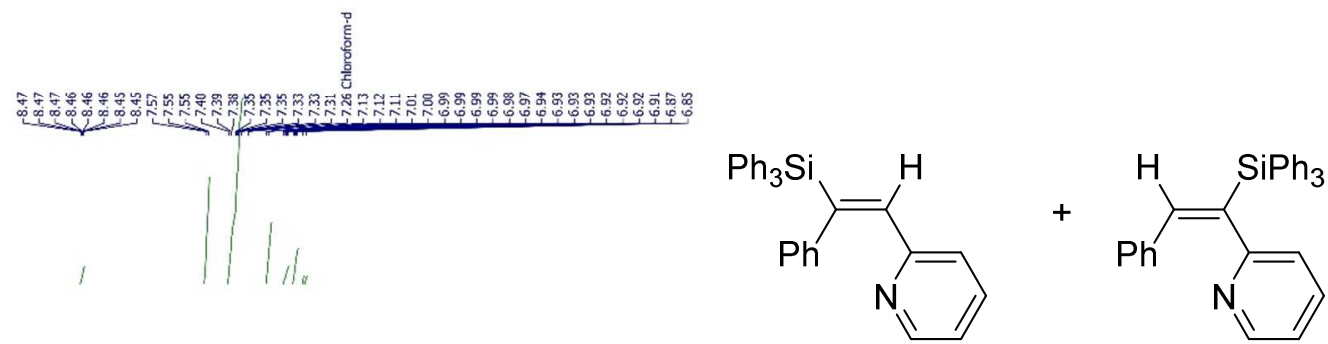

$50: 50$
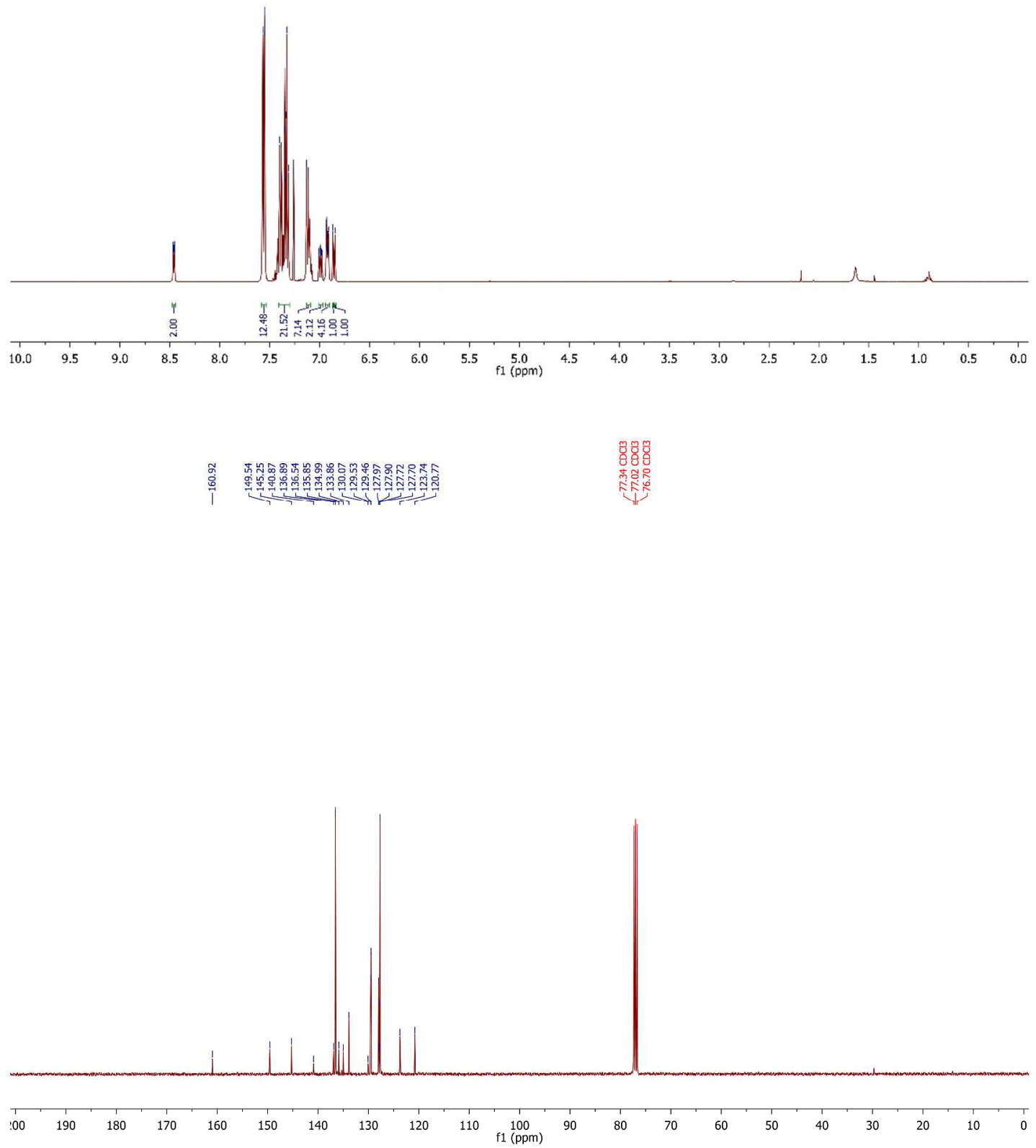
Triphenyl-[(E)-(1-(4-trifluoromethylphenyl)-2-phenylvinyl)]silane and triphenyl-[(E)-(1-phenyl-2(4-trifluoromethylphenyl)vinyl)]silane (3pa)
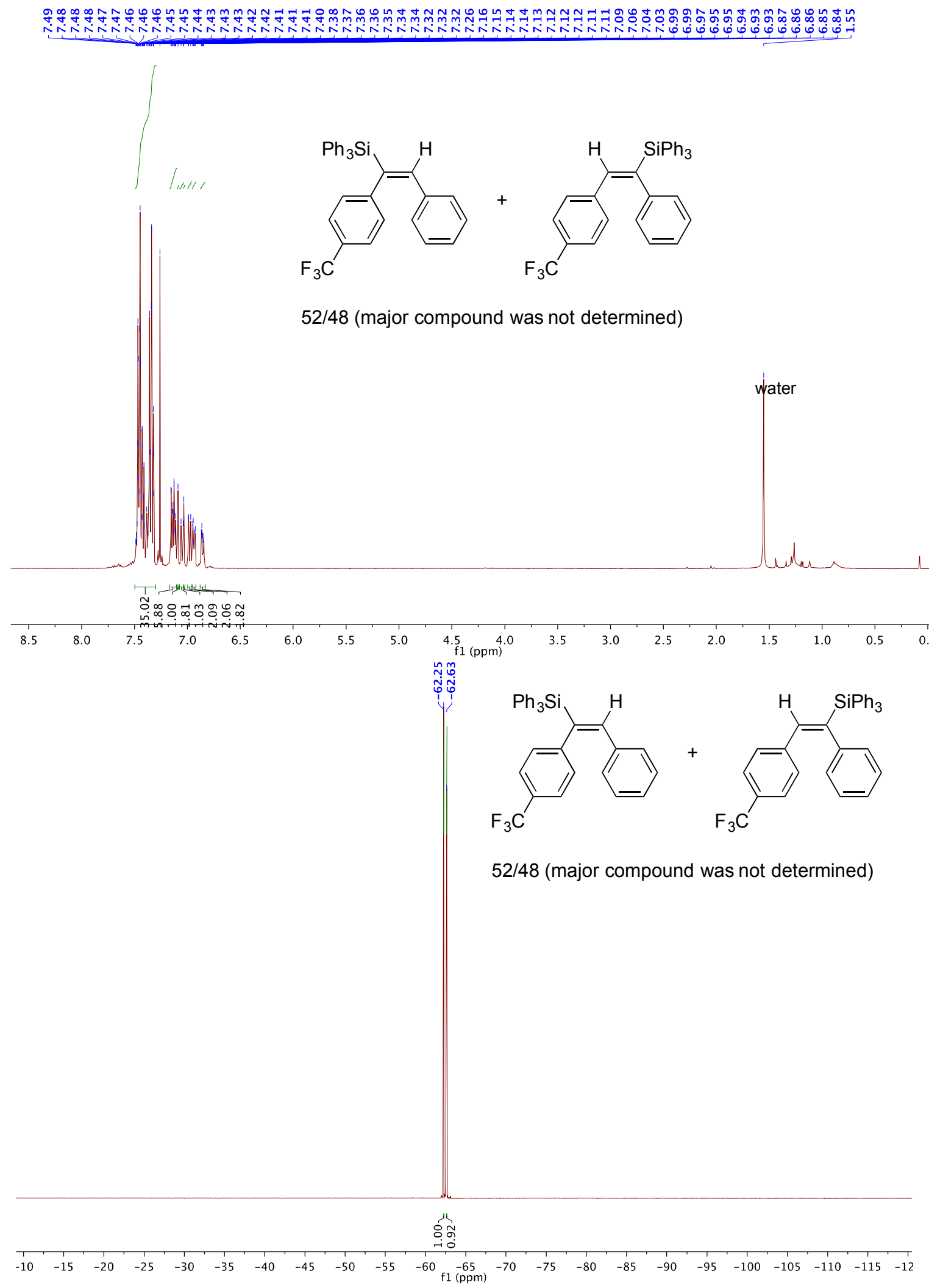


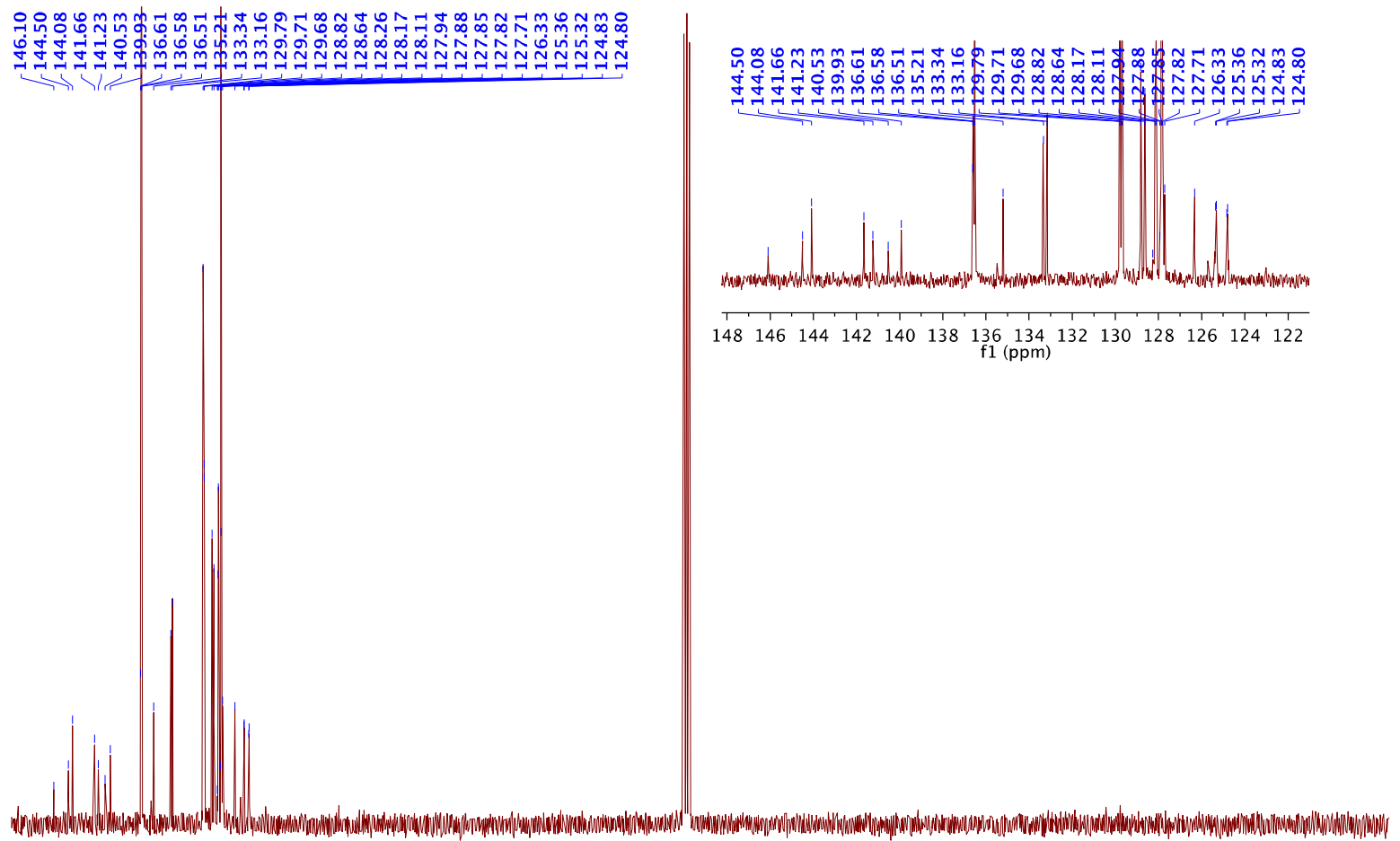

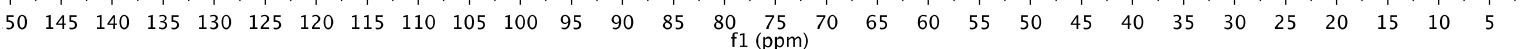




\section{(E)-Ethyl 3-phenyl-2-(triphenylsilyl)acrylate (3'qa)}
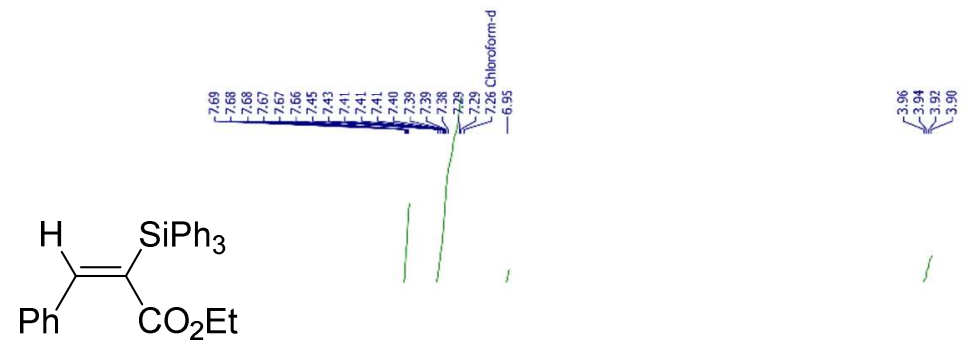

$\mathrm{Ph} \quad \mathrm{CO}_{2} \mathrm{Et}$
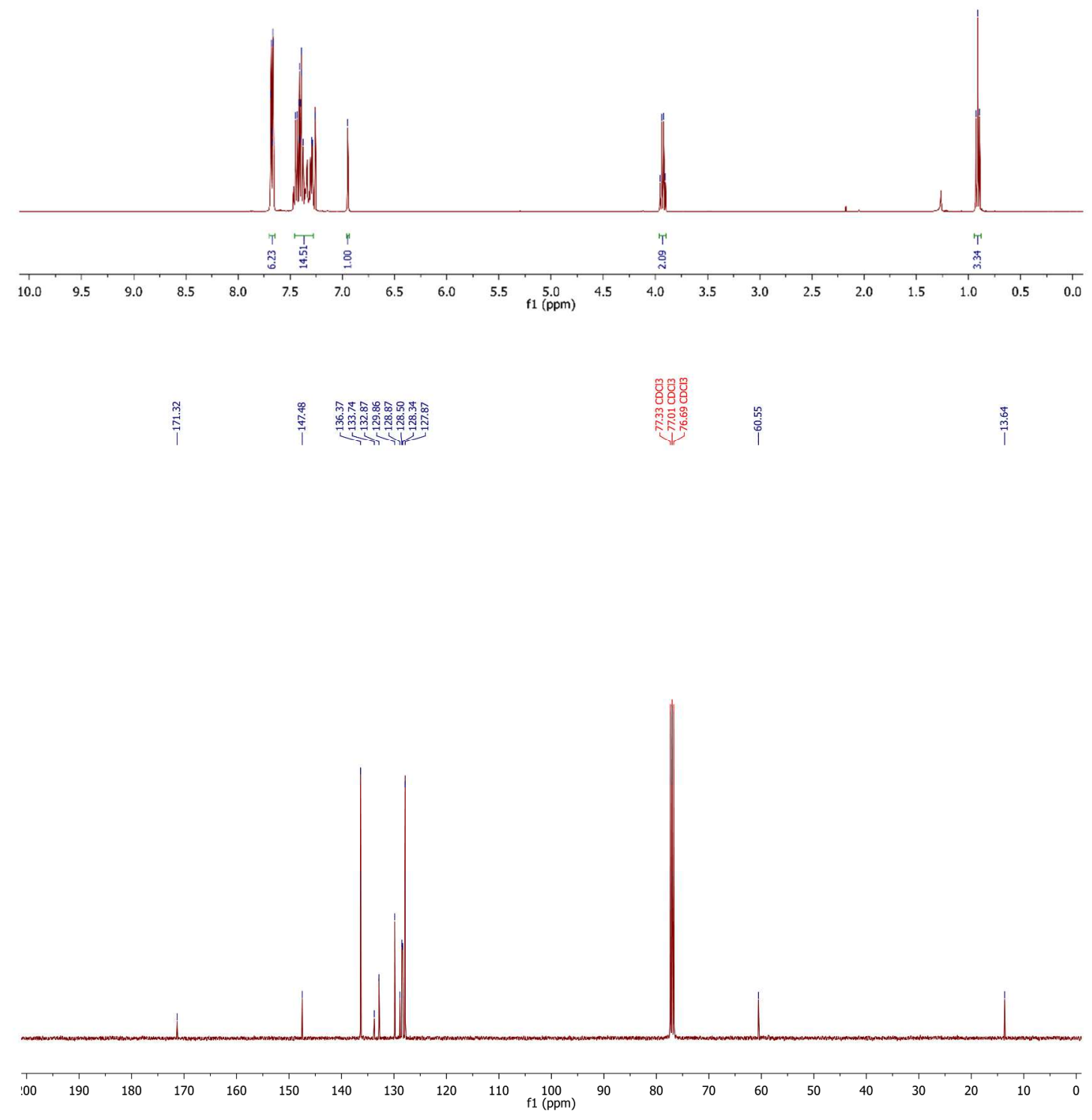
(E)-Methyl 2-(triphenylsilyl)-2-octenoate (3'ra)
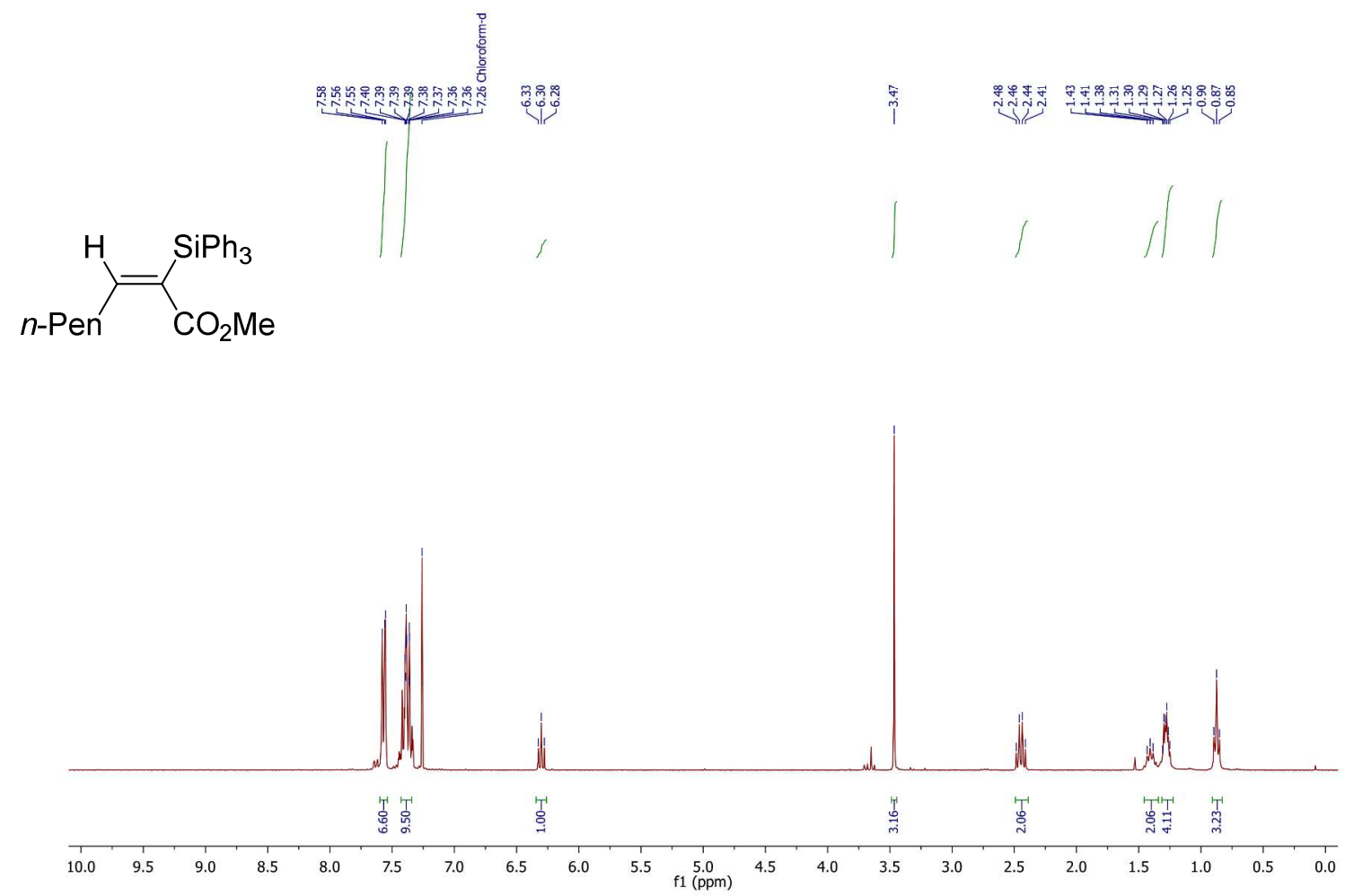

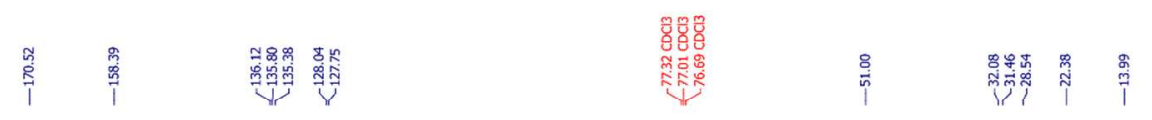

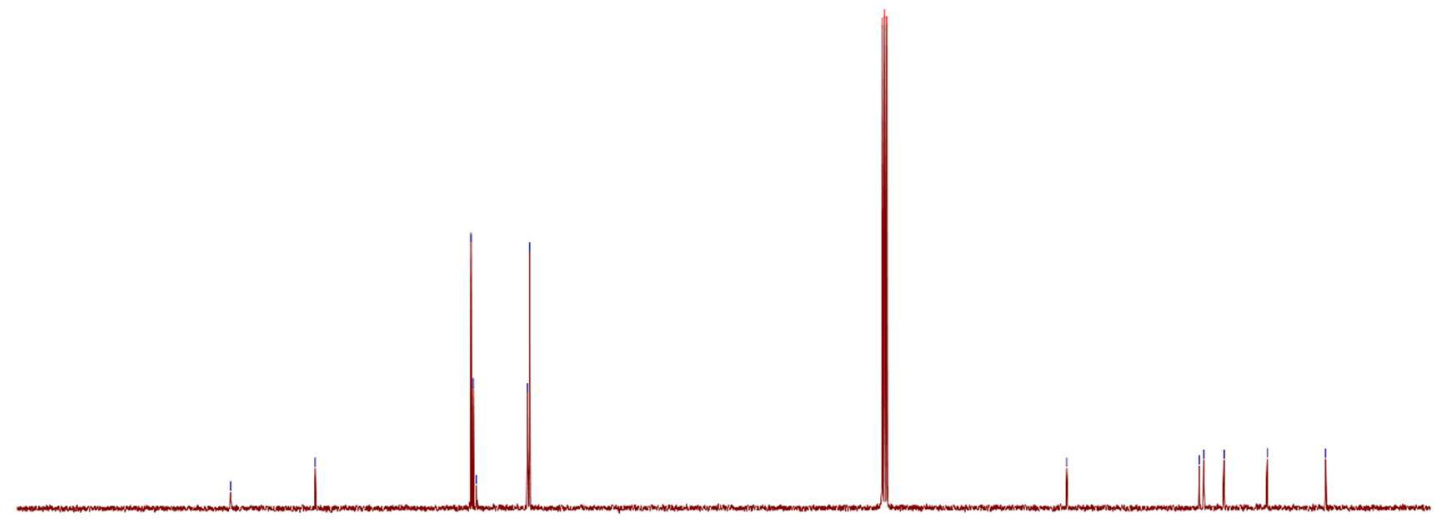

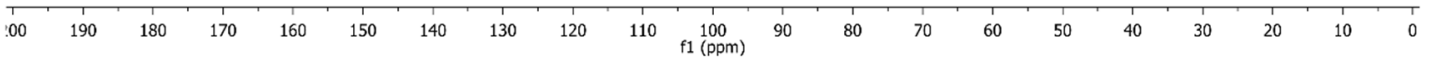


Triethoxy-[(E)-(1-phenyl-2-butylvinyl)]silane (3bb)

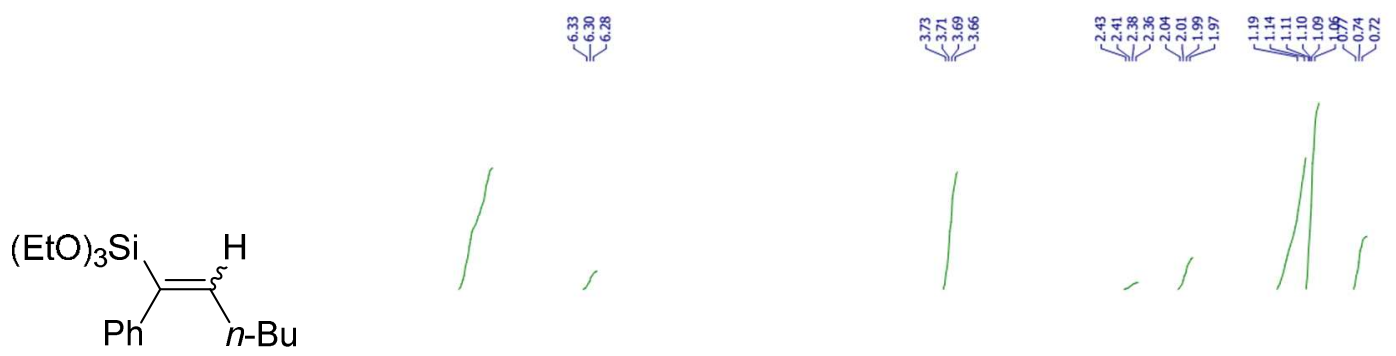

$83 / 17(E / Z)$
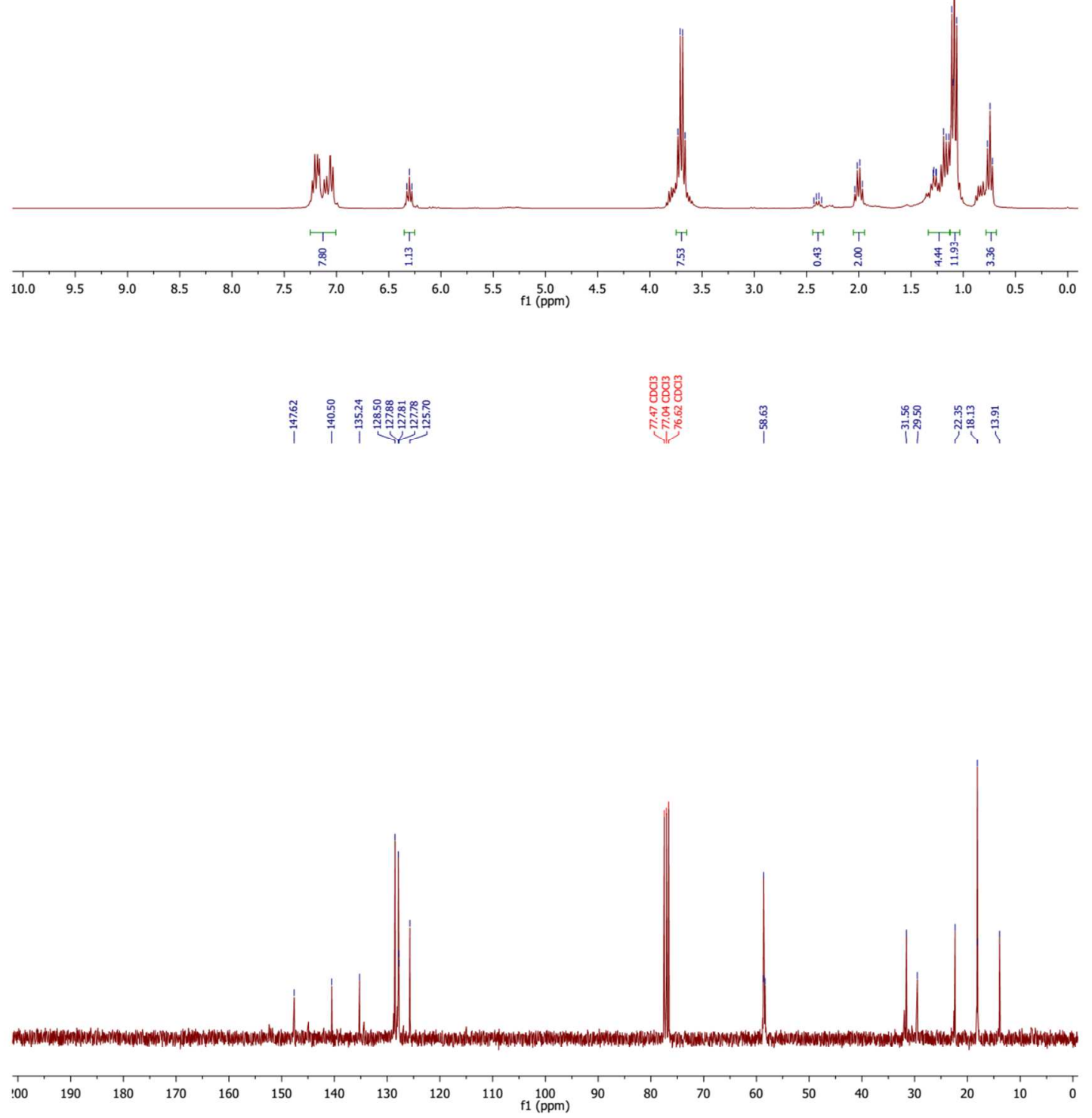
Triethoxy-[(E)-(1-(4-methoxyphenyl)-2-butylvinyl)]silane (3cb)
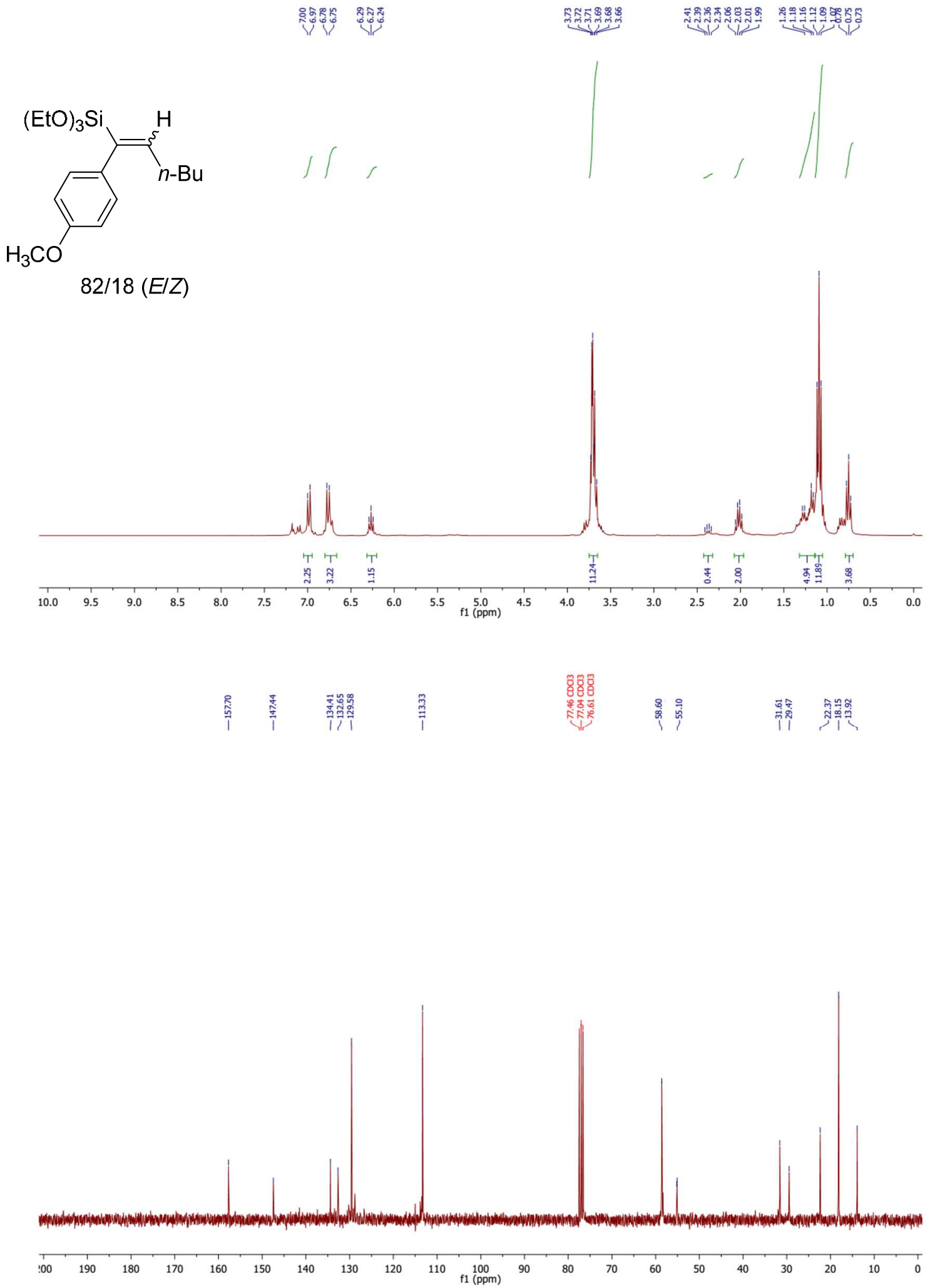
Triethoxy-[(E)-(1-(4-(trifluoromethyl)phenyl)-2-butylvinyl)]silane (3db)
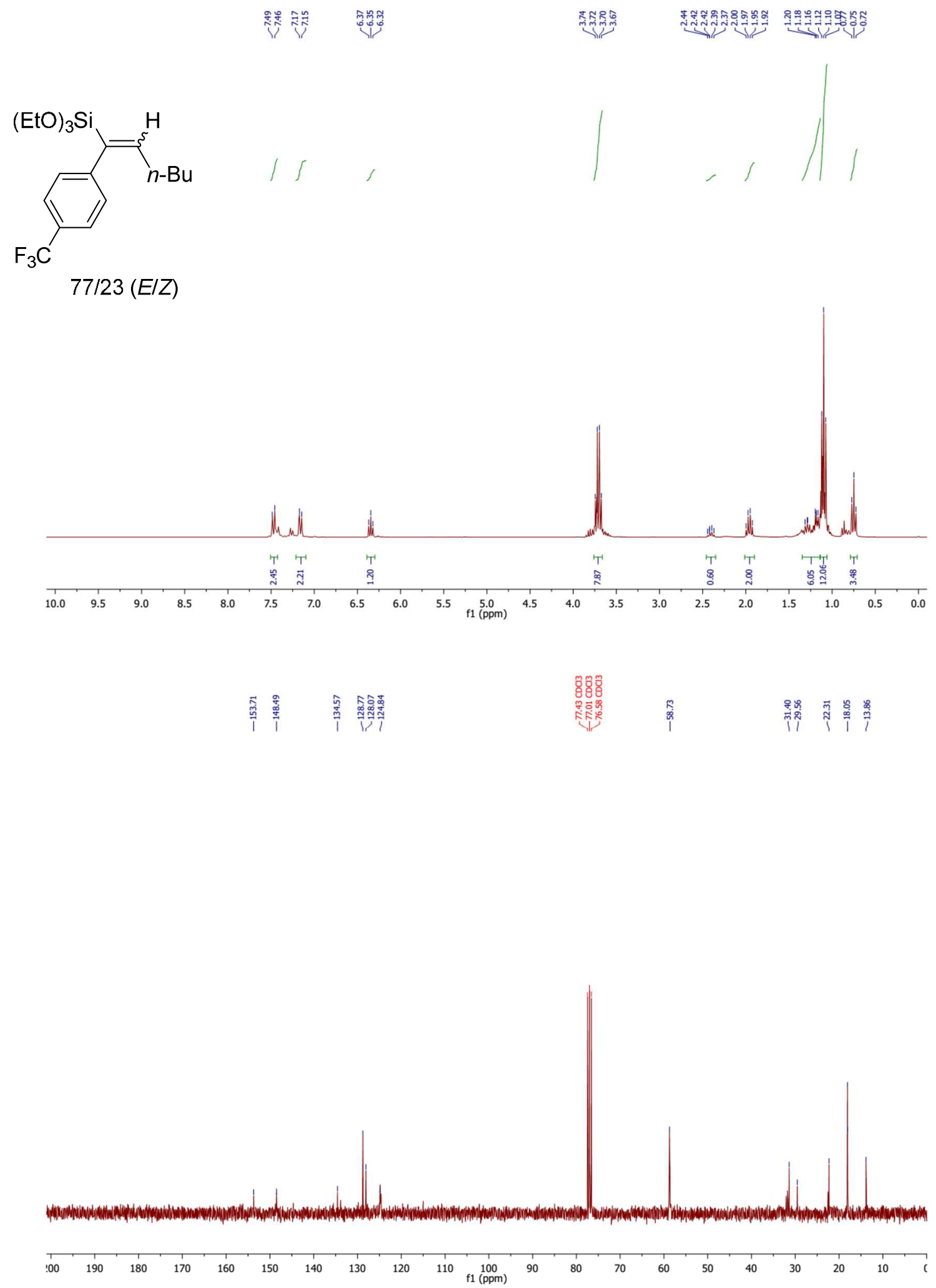
Triethoxy-[(E)-(1-phenyl-2-(trimethylsilyl)vinyl)]silane (3eb)
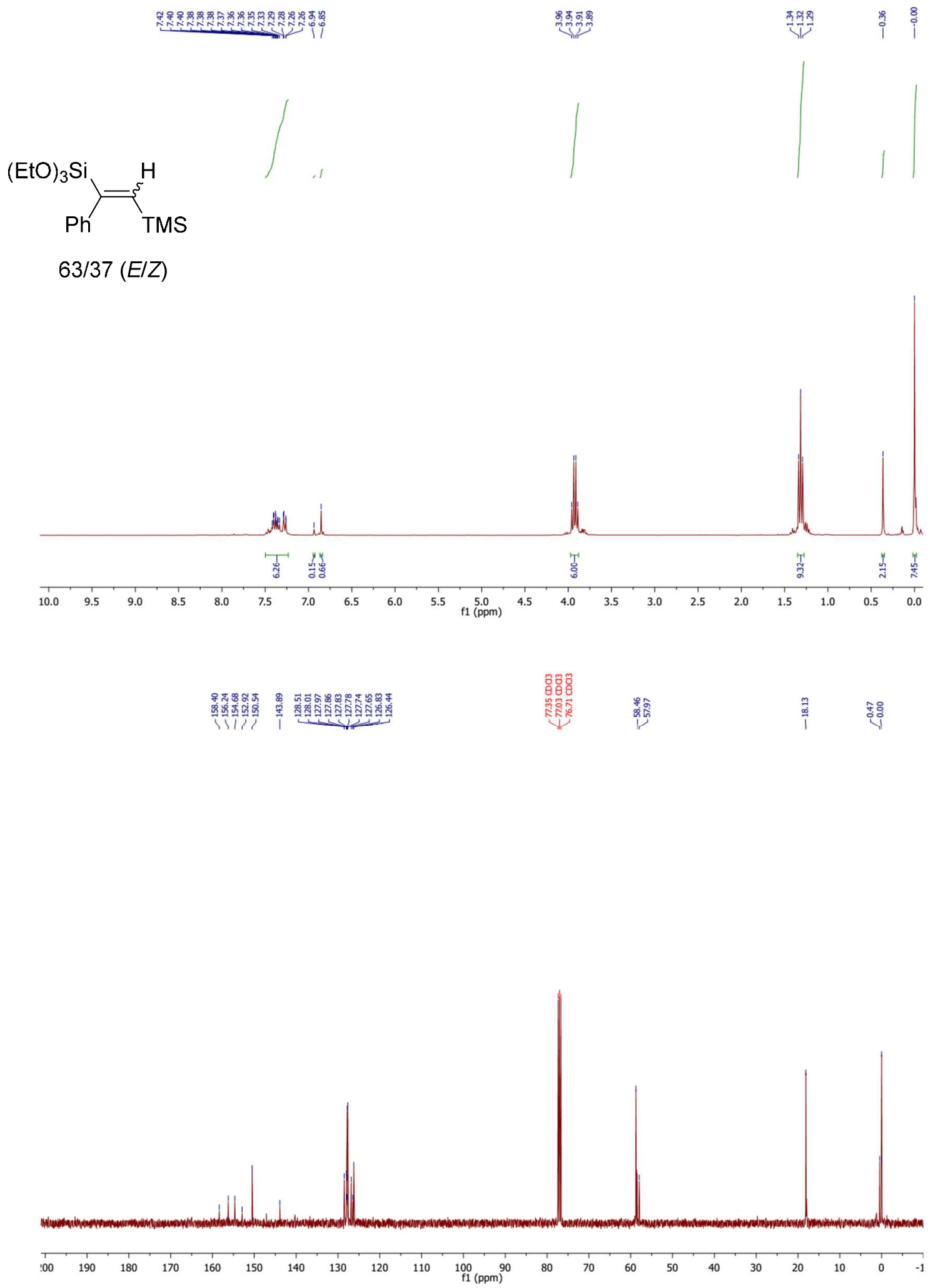
Triethoxy-[(E)-(1-(4-methoxyphenyl)-2-(trimethylsilyl)vinyl)]silane (3fb)
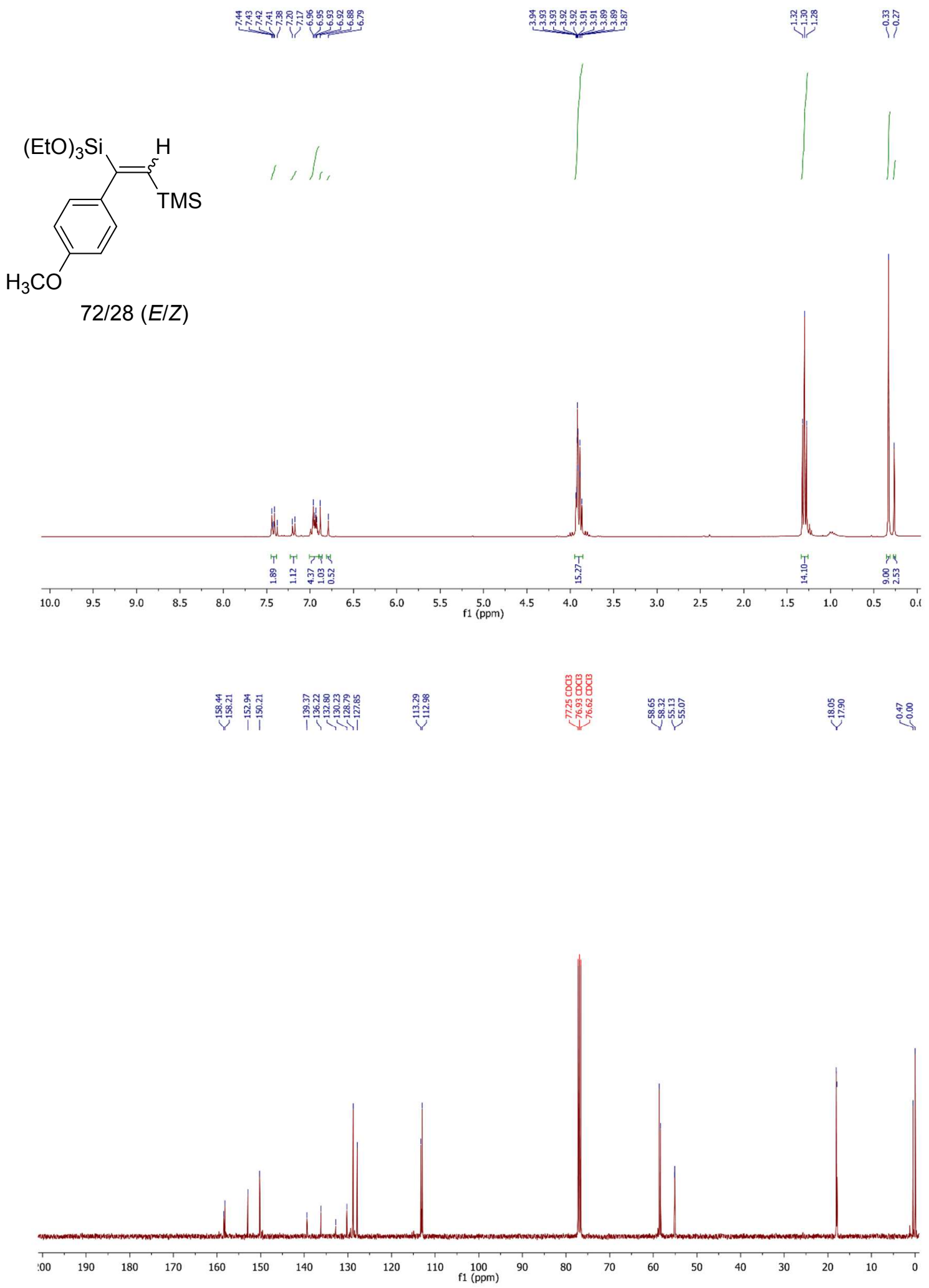
Triethoxy-[(E)-(1-(4-trifluoromethyl)phenyl-2-(trimethylsilyl)vinyl)]silane (3gb)
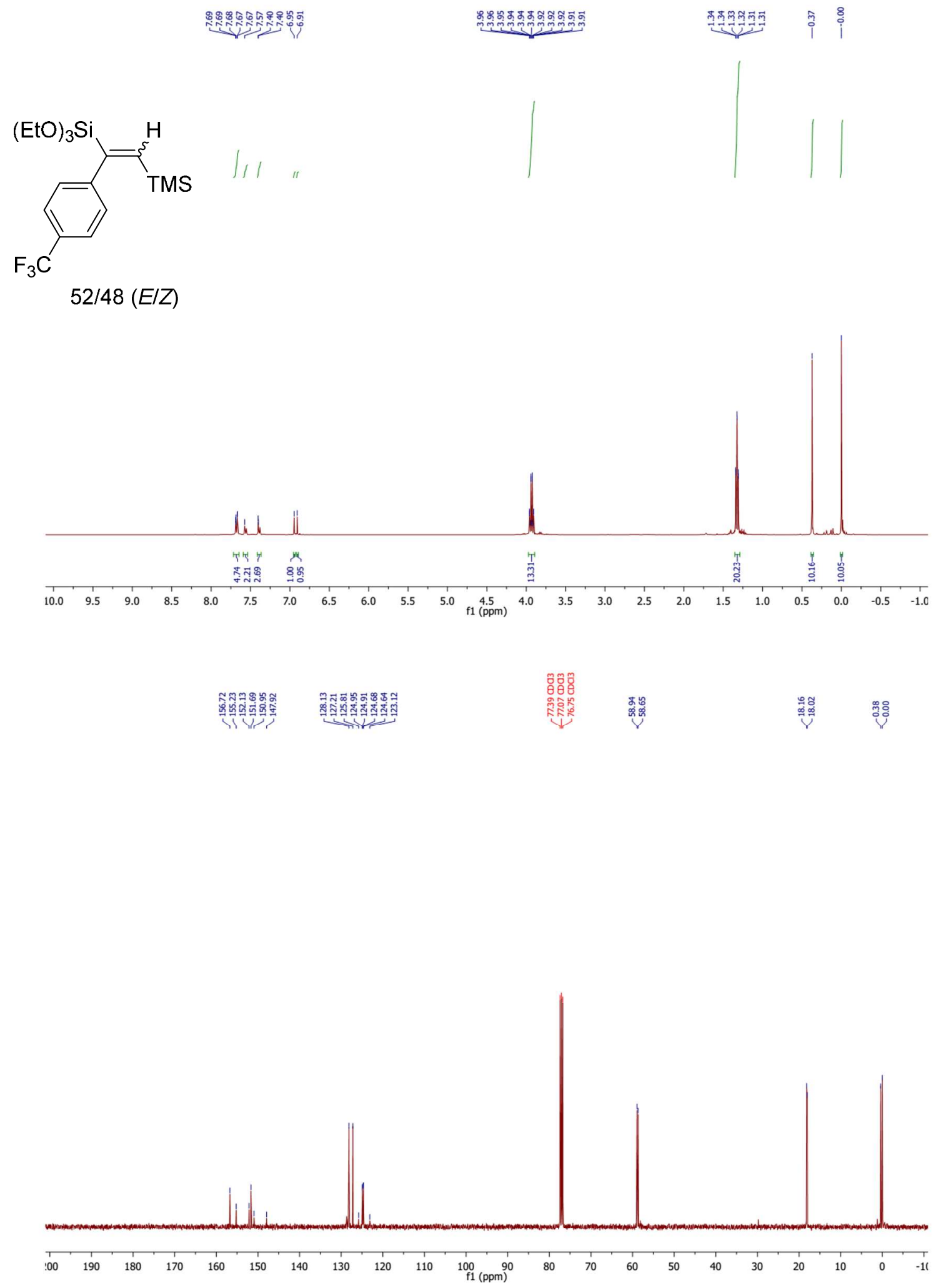
Triethoxy-[(E)-(1-(2-pyridyl)-2-(trimethylsilyl)vinyl)]silane (3jb)

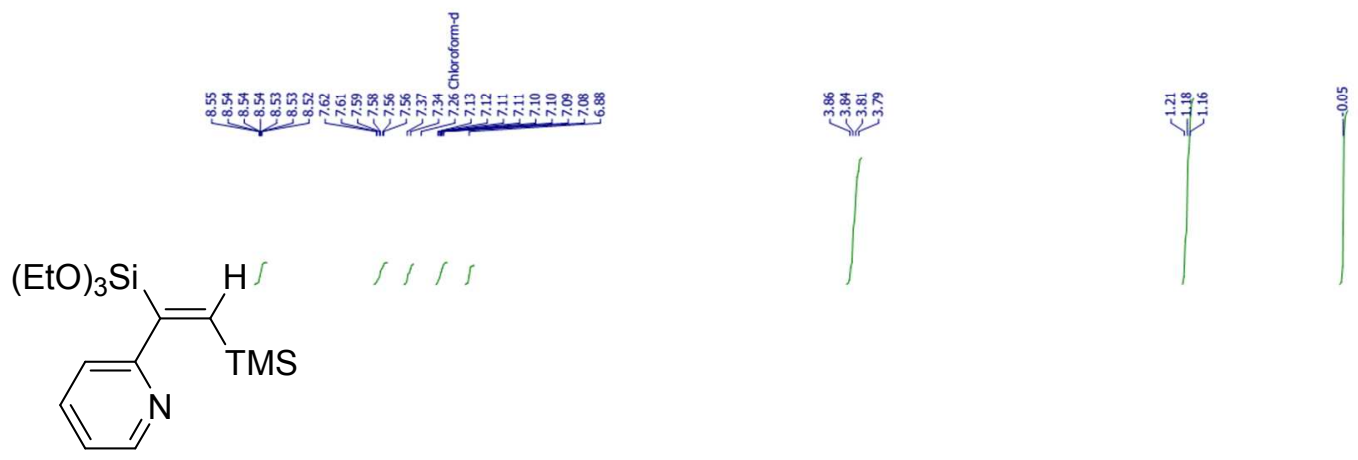

100/0 (E/Z)

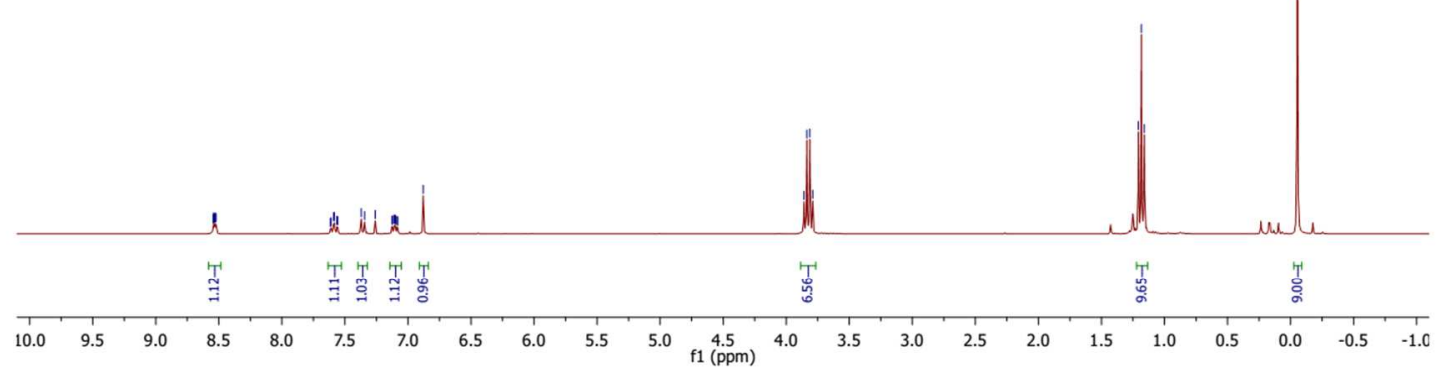

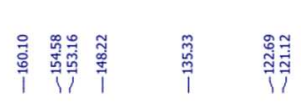

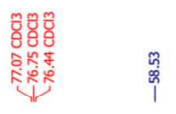

$\stackrel{\circledast}{i} \stackrel{\circ}{i}$

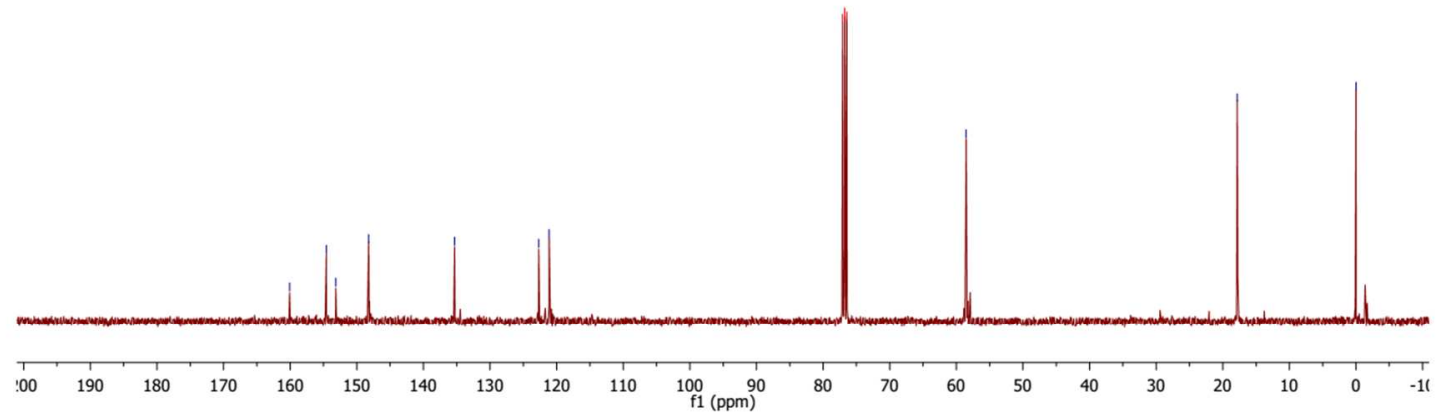


Triethoxy-[(E)-(1-(3-pyridyl)-2-(trimethylsilyl)vinyl)]silane (3kb)

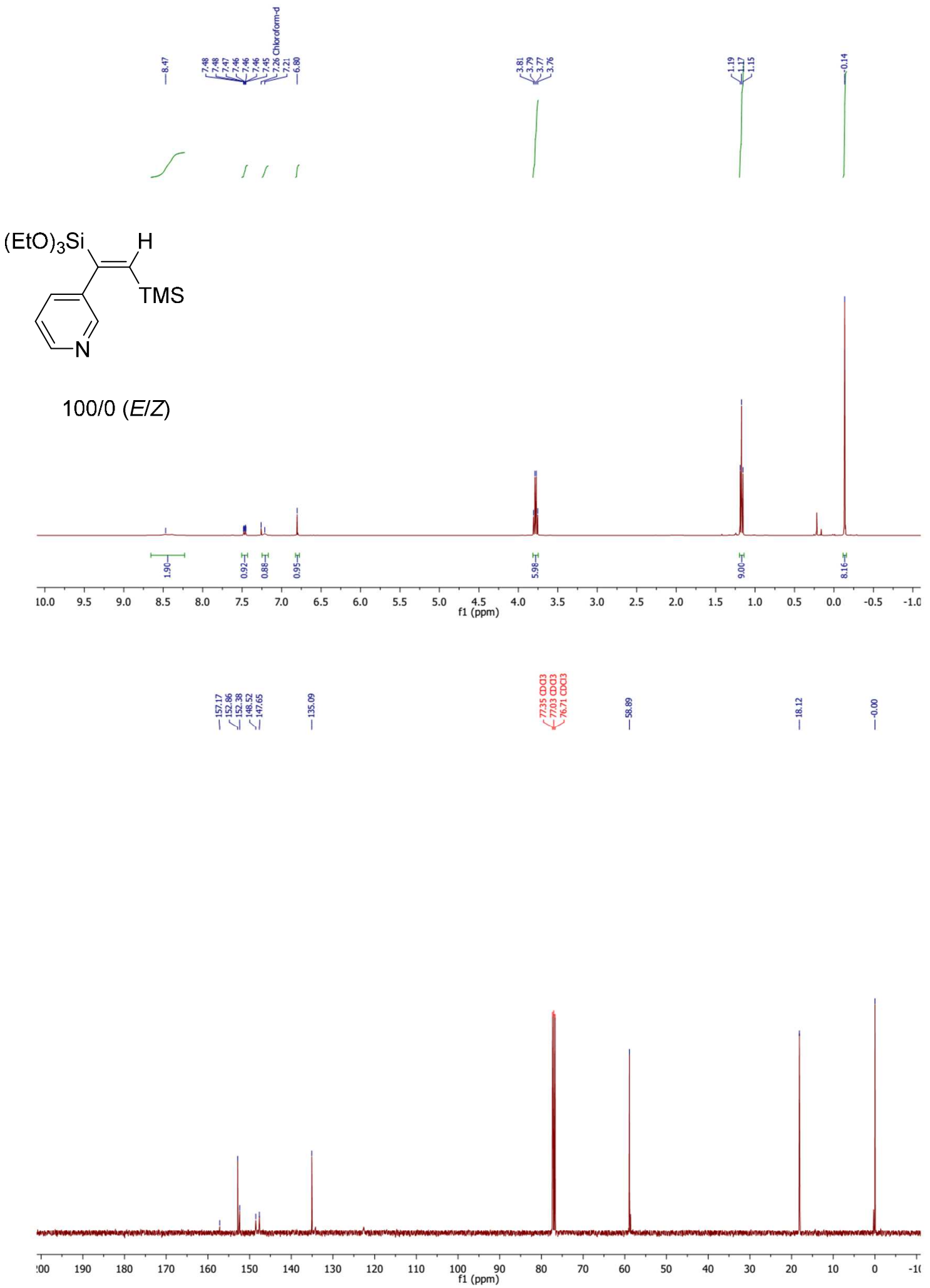


Triethoxy-[(E)-(2-(2,6-dimethylphenyl)-1-phenylvinyl)]silane (3lb)
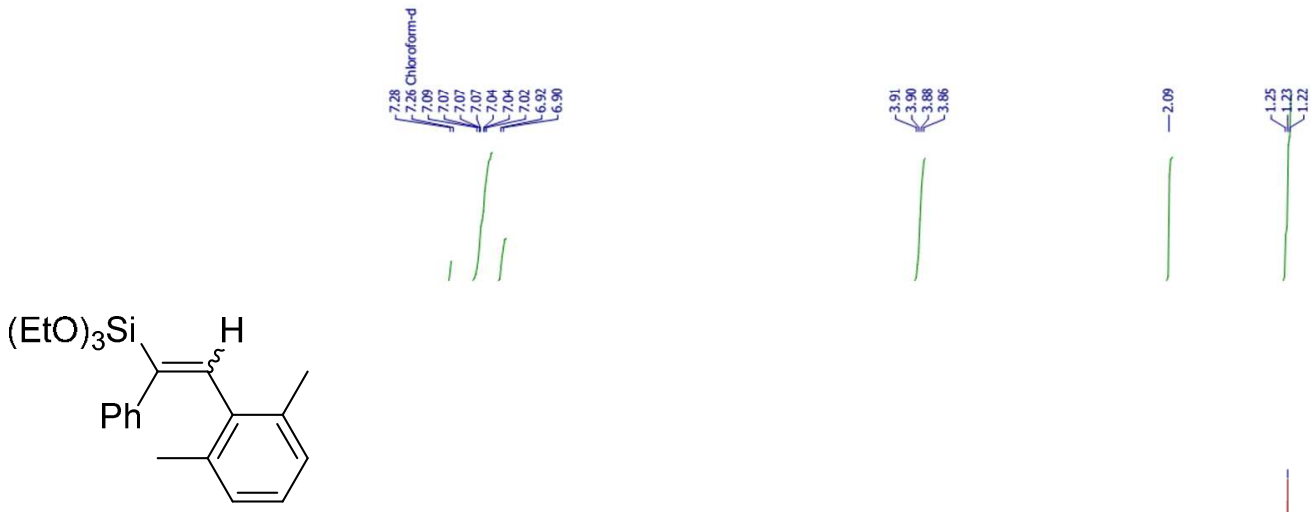

$100 / 0(E / Z)$
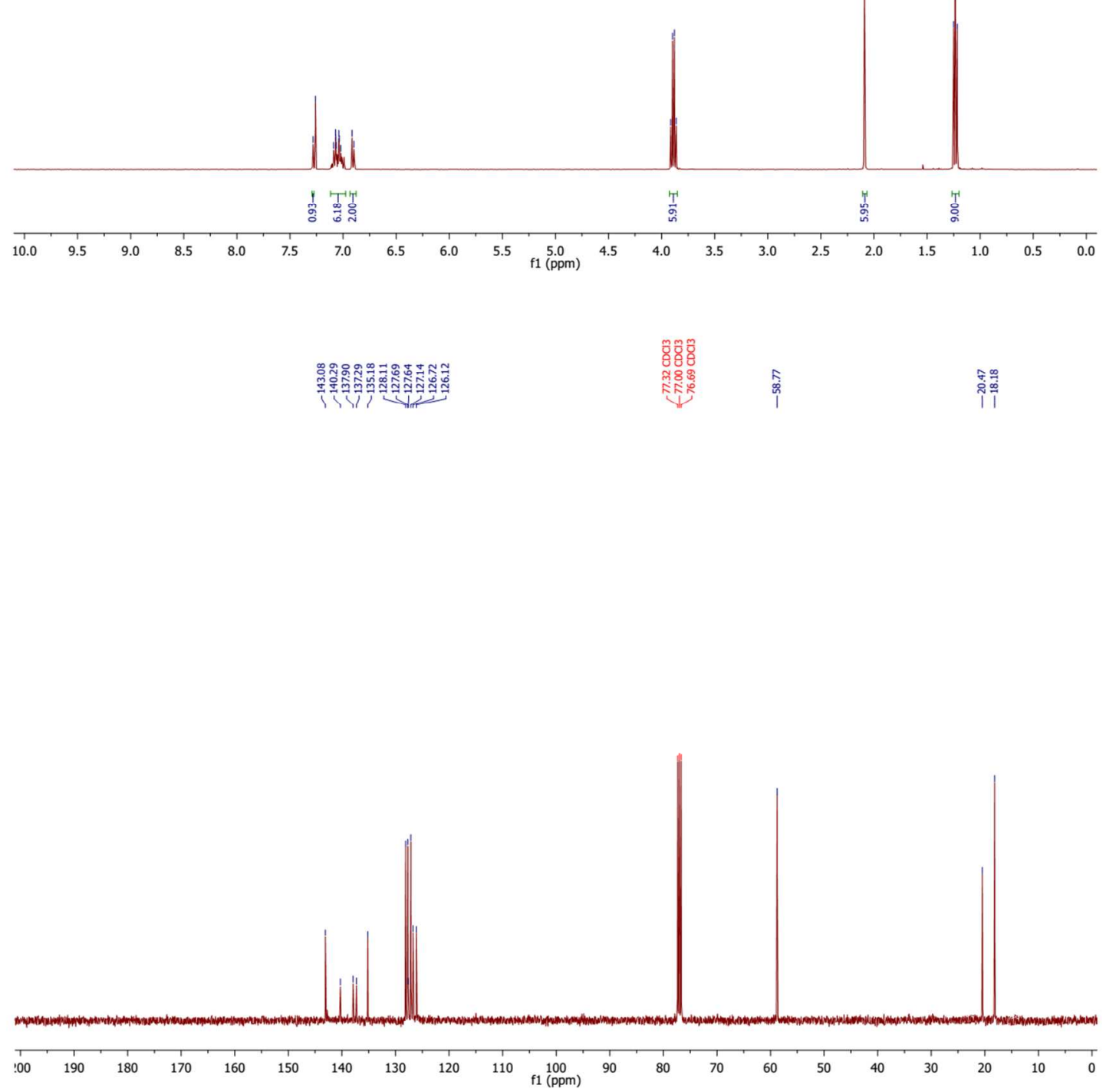
Triethoxy-[(E)-(1-(4-methoxyphenyl)-2-phenylvinyl)]silane (3nb)

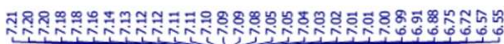

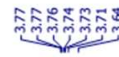

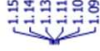
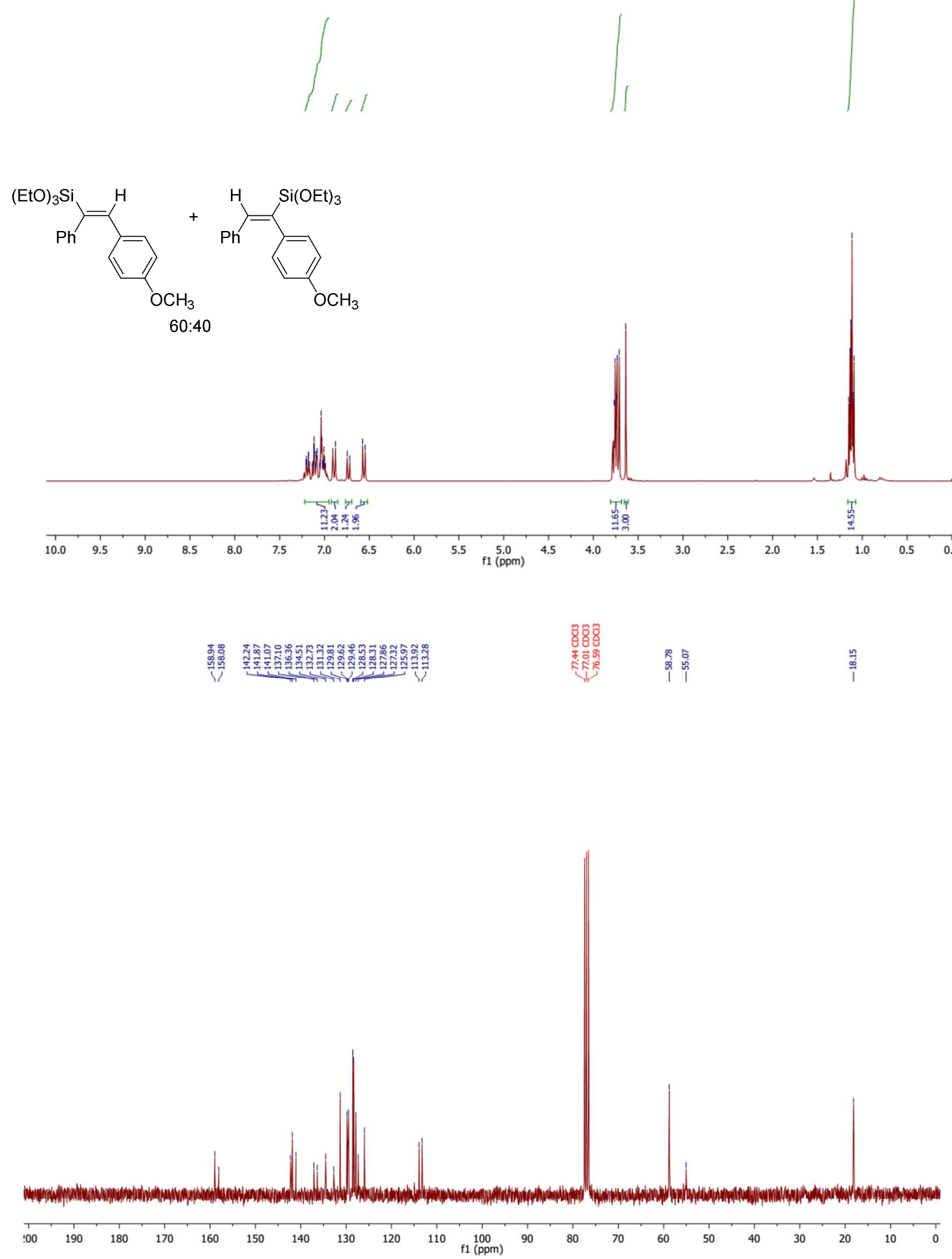


\section{(3-(2-(dimethylsilyl)phenyl)prop-1-yn-1-yl)trimethylsilane (4)}

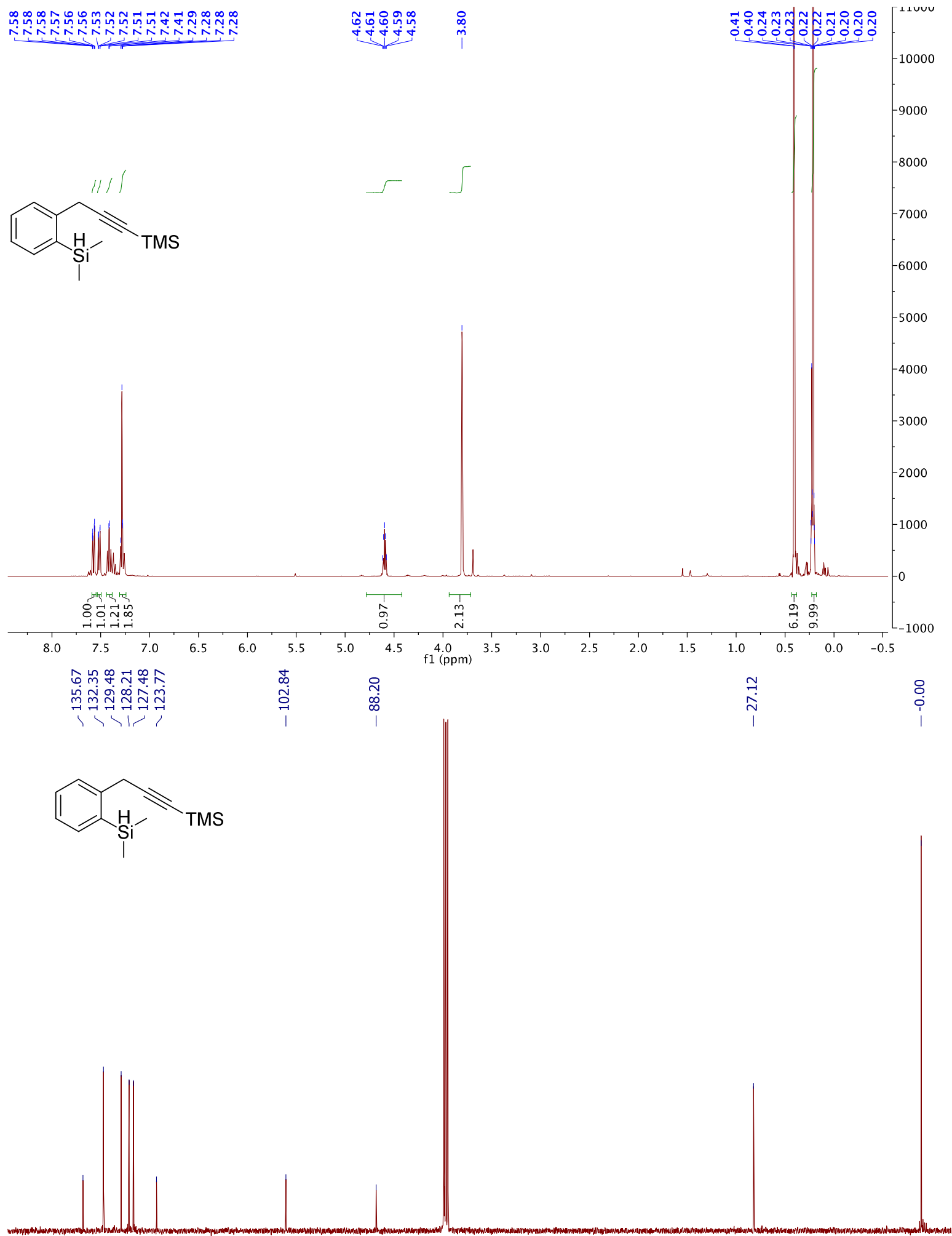

$\begin{array}{llllllllllllllllllllllllllllllll}145 & 140 & 135 & 130 & 125 & 120 & 115 & 110 & 105 & 100 & 95 & 90 & 85 & 80 & 75 & 70 & 65 & 60 & 55 & 50 & 45 & 40 & 35 & 30 & 25 & 20 & 15 & 10 & 5 & 0\end{array}$ 


\section{1,1-dimethyl-2-((trimethylsilyl)methyl)-1H-benzo[b]silole (5)}

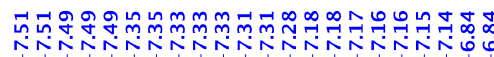<smiles>C[Si]1(C)C(CS(C)(C)(C)=O)=Cc2ccccc21</smiles><smiles>CC1CCCC1C</smiles>
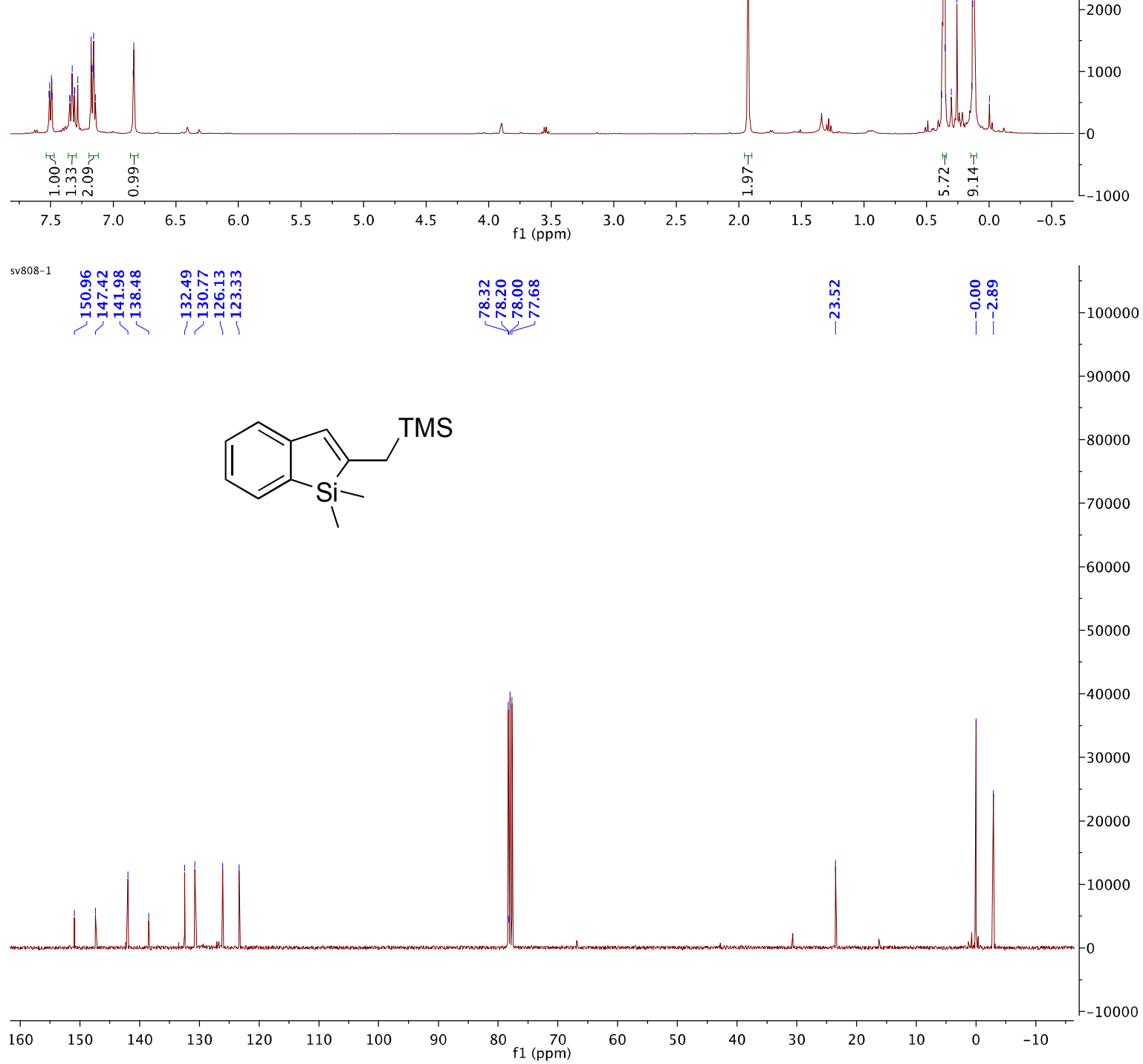

TMS 
dihydridocobalt(III) complex (6) : ${ }^{1} \mathrm{H},{ }^{13} \mathrm{C},{ }^{31} \mathrm{P},{ }^{29} \mathrm{Si}$ NMR analysis

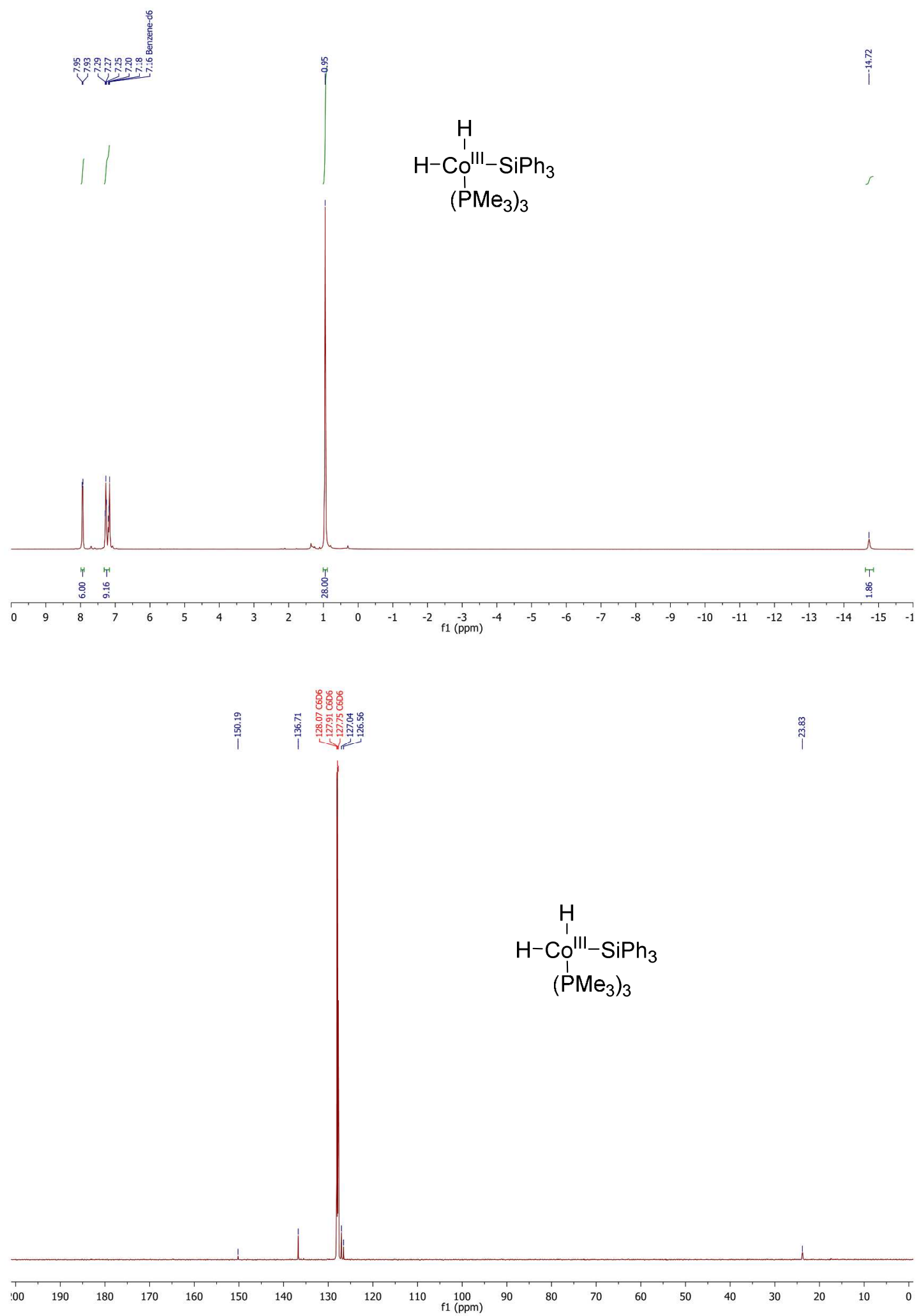




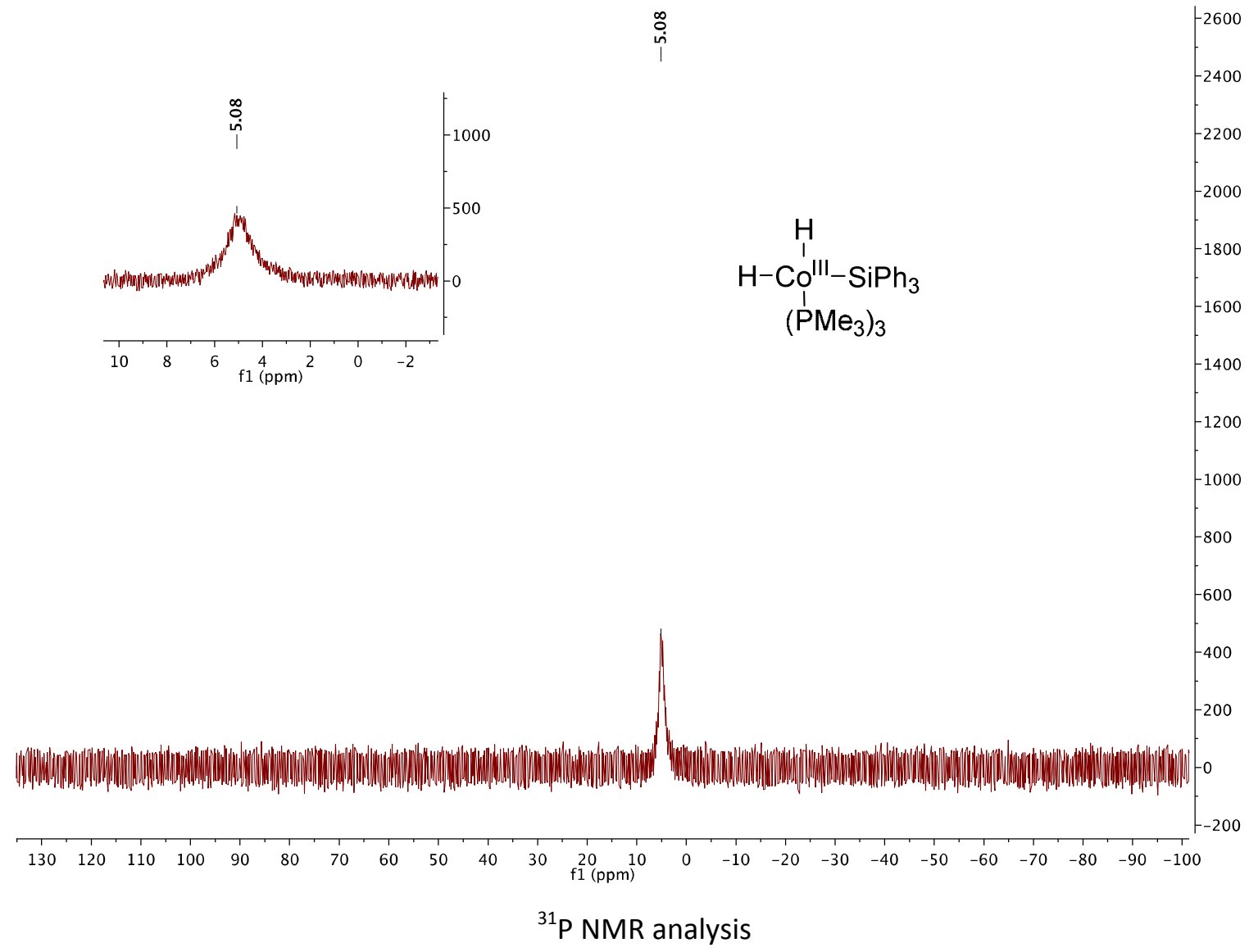

$\stackrel{\infty}{\circ}$ 
ARHDHCO
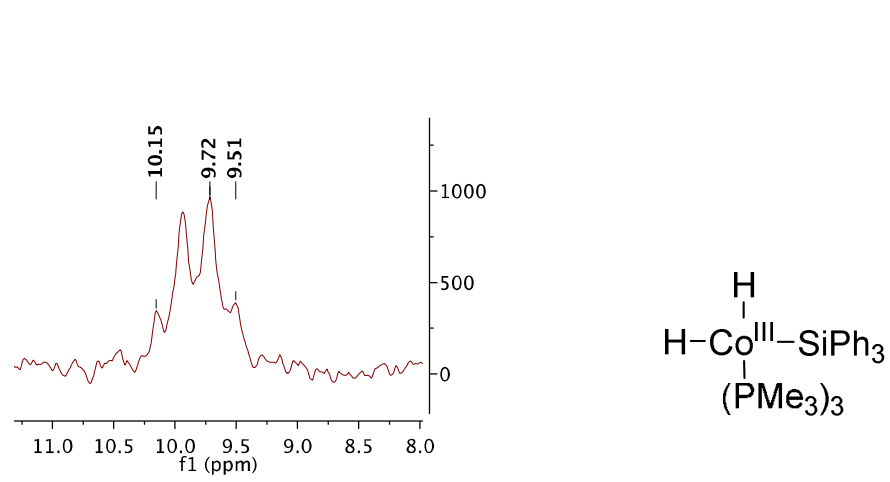

곡
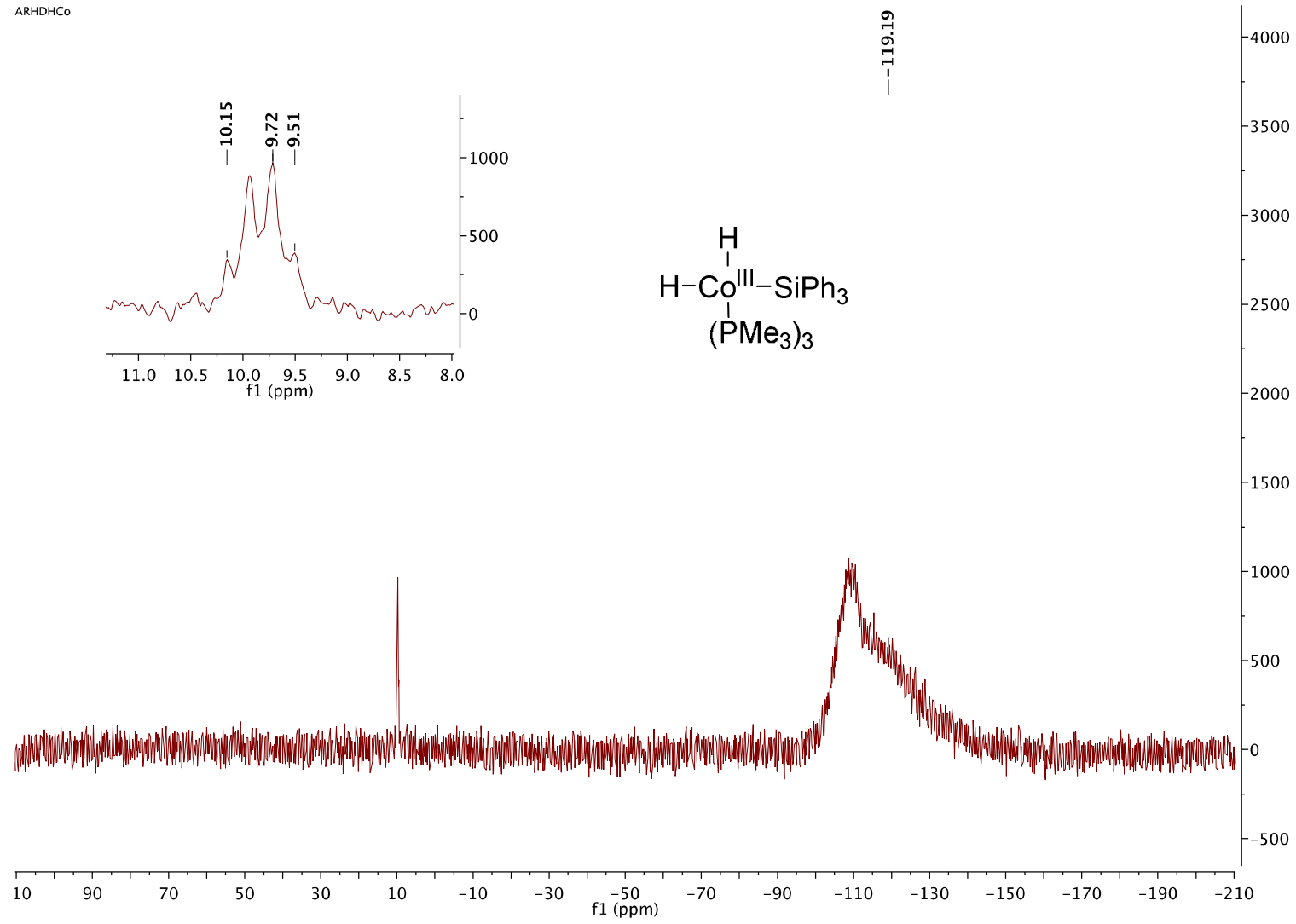

${ }^{29} \mathrm{Si}$ NMR analysis : observed signal is a quadruplet with a ${ }^{2} \mathrm{~J}_{\mathrm{Si}-\mathrm{p}}$ coupling constant of $24,79 \mathrm{~Hz}$ 